\title{
SOME LINEAR PARABOLIC SYSTEM IN BESOV SPACES
}

\author{
EWA ZADRZYŃSKA \\ Faculty of Mathematics and Information Sciences \\ Warsaw University of Technology \\ pl. Politechniki 1, 00-661 Warszawa, Poland \\ E-mail: emzad@impan.gov.pl \\ WOJCIECH M. ZAJĄCZKOWSKI \\ Institute of Mathematics, Polish Academy of Sciences \\ Sniadeckich 8, 00-950 Warszawa, Poland \\ E-mail:wz@impan.gov.pl
}

\begin{abstract}
We study the solvability in anisotropic Besov spaces $B_{p, q}^{\frac{\sigma}{2}, \sigma}\left(\Omega^{T}\right), \sigma \in \mathbb{R}_{+}, p, q \in(1, \infty)$ of an initial-boundary value problem for the linear parabolic system which arises in the study of the compressible Navier-Stokes system with boundary slip conditions.

The proof of existence of a unique solution in $B_{p, q}^{\frac{\sigma}{2}+1, \sigma+2}\left(\Omega^{T}\right)$ is divided into three steps:

$1^{\circ}$ First the existence of solutions to the problem with vanishing initial conditions is proved by applying the Paley-Littlewood decomposition and some ideas of Triebel. All considerations in this step are performed on the Fourier transform of the solution.

$2^{\circ}$ Applying the regularizer technique the existence is proved in a bounded domain.

$3^{\circ}$ The problem with nonvanishing initial data is solved by an appropriate extension of initial data.
\end{abstract}

1. Introduction. In some cases the analysis of the compressible Navier-Stokes equations requires the study of the following linear parabolic system (see for example [7])

$$
u_{t}-\operatorname{div} \mathbb{D}(u)=f \quad \text { in } \Omega^{T}=(0, T) \times \Omega,
$$

where $u(t, x)=\left(u_{1}(t, x), u_{2}(t, x), u_{3}(t, x)\right)$ is an unknown function, $t \in(0, T), 0<T<\infty$, $x=\left(x_{1}, x_{2}, x_{3}\right) \in \Omega \subset \mathbb{R}^{3}$, and $\Omega$ is a bounded domain with boundary $S$.

2000 Mathematics Subject Classification: Primary 35K45, 35K50; Secondary 35K40.

Key words and phrases: linear parabolic systems, boundary slip conditions, Besov regularity of solutions.

Research partially supported by MNiSW grant no 1P03A 02130.

The paper is in final form and no version of it will be published elsewhere. 
Moreover,

$$
\mathbb{D}(u)=\left\{\mu\left(\partial_{x_{i}} u_{j}+\partial_{x_{j}} u_{i}\right)+(\nu-\mu) \operatorname{div} u \delta_{i j}\right\}_{i, j=1,2,3}
$$

and $\mu, \nu$ are constant viscosity coefficients. From thermodynamic considerations it follows that $\nu>\frac{1}{3} \mu>0$.

We associate with system (1.1) the following boundary slip conditions and initial condition:

$$
\begin{gathered}
\bar{\tau}_{\alpha} \cdot \mathbb{D}(u) \bar{n}=b_{\alpha}, \quad \alpha=1,2 \quad \text { on } S^{T}=(0, T) \times S, \\
u \cdot \bar{n}=b_{3} \quad \text { on } S^{T}, \\
\left.u\right|_{t=0}=u_{0} \quad \text { in } \Omega,
\end{gathered}
$$

where $\bar{n}, \bar{\tau}_{\alpha}, \alpha=1,2$, are unit orthonormal vectors such that $\bar{n}$ is the outward normal vector and $\bar{\tau}_{1}, \bar{\tau}_{2}$ are tangent to $S$. By dot we denote the scalar product in $\mathbb{R}^{3}$.

In this paper we shall examine the solvability of problem (1.1)-(1.4) in anisotropic Besov spaces $B_{p, q}^{\frac{\sigma}{2}, \sigma}\left(\Omega^{T}\right), \sigma \in \mathbb{R}_{+}, p, q \in(1, \infty)$ (see Section 2$)$.

We treat problem (1.1)-(1.4) as a model problem because the methods used in the paper can be extended to other boundary problems and to other more general linear parabolic equations and systems.

In order to formulate the main result of the paper we need some compatibility conditions on the data.

Let $u_{0} \in B_{p, q}^{\sigma+2-\frac{2}{p}}(\Omega)$. Assume that $\tilde{u}_{0} \in B_{p, q}^{\frac{\sigma}{2}+1, \sigma+2}\left(\Omega^{T}\right)$ is an extension of $u_{0}$ on $(0, T) \times \Omega$ such that $\left.\tilde{u}_{0}\right|_{t=0}=u_{0}$. Next, let $b_{k} \in B_{p, q}^{\left(\sigma+1-\frac{1}{p}\right) / 2, \sigma+1-\frac{1}{p}}\left(S^{T}\right), k=1,2, b_{3} \in$ $B_{p, q}^{\frac{\sigma}{2}+1-\frac{1}{2 p}, \sigma+2-\frac{1}{p}}\left(S^{T}\right)$. We extend $b_{k}(k=1,2,3)$ onto a neighbourhood $\Omega^{\prime} \subset \mathbb{R}^{3}$ of $S$ to functions $\tilde{b}_{k} \in B_{p, q}^{(\sigma+1) / 2, \sigma+1}\left(\Omega^{\prime} T\right), k=1,2$ and $\tilde{b}_{3} \in B_{p, q}^{\frac{\sigma}{2}+1, \sigma+2}\left(\Omega^{\prime T}\right)$ satisfying: $\left.\tilde{b}_{k}\right|_{S}=b_{k}$, $k=1,2,3$.

Then, we define the functions

$$
\begin{aligned}
& f_{0}=f-\left(\tilde{u}_{0 t}-\operatorname{div} \mathbb{D}\left(\tilde{u}_{0}\right)\right), \\
& b_{0 k}=\tilde{b}_{k}-\bar{\tau}_{k} \cdot \mathbb{D}\left(\tilde{u}_{0}\right) \bar{n}, \quad k=1,2, \\
& b_{03}=\tilde{b}_{3}-\tilde{u}_{0} \cdot \bar{n} .
\end{aligned}
$$

Assumption 1.1. We assume that:

$$
\begin{gathered}
\int_{0}^{T} \frac{\left\|\partial_{t}^{m_{1}} f_{0}\right\|_{L_{p}\left(\Omega^{t}\right)}^{q}}{t^{1+q \lambda_{1}}} d t<\infty, \quad \int_{0}^{T} \frac{\left\|\left.\partial_{t}^{m_{2}} b_{0 k}\right|_{S}\right\|_{L_{p}\left(S^{t}\right)}^{q}}{t^{1+q \lambda_{2}}} d t<\infty, \quad k=1,2, \\
\int_{0}^{T} \frac{\left\|\left.\partial_{t}^{m_{3}} b_{03}\right|_{S}\right\|_{L_{p}\left(S^{t}\right)}^{q}}{t^{1+q \lambda_{3}}} d t<\infty
\end{gathered}
$$

where

$$
\begin{gathered}
m_{1}= \begin{cases}{\left[\frac{\sigma}{2}\right]} & \text { if } \frac{\sigma}{2} \text { is noninteger, } \\
\frac{\sigma}{2}-1 & \text { if } \frac{\sigma}{2} \in \mathbb{N},\end{cases} \\
m_{2}= \begin{cases}{\left[\frac{\sigma}{2}+\frac{1}{2}-\frac{1}{2 p}\right]} & \text { if } \frac{\sigma}{2}+\frac{1}{2}-\frac{1}{2 p} \text { is noninteger, } \\
\frac{\sigma}{2}-\frac{1}{2}-\frac{1}{2 p} & \text { if } \frac{\sigma}{2}+\frac{1}{2 p}-\frac{1}{2 p} \in \mathbb{N},\end{cases}
\end{gathered}
$$




$$
\begin{gathered}
m_{3}= \begin{cases}{\left[\frac{\sigma}{2}+1-\frac{1}{2 p}\right]} & \text { if } \frac{\sigma}{2}+1-\frac{1}{2 p} \text { is noninteger, } \\
\frac{\sigma}{2}-\frac{1}{2 p} & \text { if } \frac{\sigma}{2}+1-\frac{1}{2 p} \in \mathbb{N},\end{cases} \\
\lambda_{1}= \begin{cases}\frac{\sigma}{2}-\left[\frac{\sigma}{2}\right] & \text { if } \frac{\sigma}{2} \text { is noninteger, } \\
1 & \text { if } \frac{\sigma}{2} \in \mathbb{N},\end{cases} \\
\lambda_{2}= \begin{cases}\frac{\sigma}{2}+\frac{1}{2}-\frac{1}{2 p}-\left[\frac{\sigma}{2}+\frac{1}{2}-\frac{1}{2 p}\right] & \text { if } \frac{\sigma}{2}+\frac{1}{2}-\frac{1}{2 p} \text { is noninteger } \\
1 & \text { if } \frac{\sigma}{2}+\frac{1}{2}-\frac{1}{2 p} \in \mathbb{N},\end{cases} \\
\lambda_{3}= \begin{cases}\frac{\sigma}{2}+1-\frac{1}{2 p}-\left[\frac{\sigma}{2}+1-\frac{1}{2 p}\right] & \text { if } \frac{\sigma}{2}+1-\frac{1}{2 p} \text { is noninteger, } \\
1 & \text { if } \frac{\sigma}{2}+1-\frac{1}{2 p} \in \mathbb{N} .\end{cases}
\end{gathered}
$$

Assumption 1.2. We assume the following compatibility conditions:

$$
\begin{gathered}
\left.D_{x}^{\alpha} \partial_{t}^{i} f_{0}\right|_{t=0}=0 \quad \text { for }|\alpha|+2 i \leq l_{1}, \\
\left.D_{x}^{\alpha} \partial_{t}^{i} b_{0 k}\right|_{t=0}=0 \quad \text { for } k=1,2, \quad|\alpha|+2 i \leq l_{2}, \\
\left.D_{x}^{\alpha} \partial_{t}^{i} b_{03}\right|_{t=0}=0 \quad \text { for }|\alpha|+2 i \leq l_{3},
\end{gathered}
$$

where

$$
\begin{gathered}
l_{1}= \begin{cases}\sigma-\frac{2}{p}-1 & \text { if } \sigma-\frac{2}{p} \in \mathbb{N}, \\
{\left[\sigma-\frac{2}{p}\right]} & \text { if } \sigma-\frac{2}{p}>0 \text { and } \sigma-\frac{2}{p} \notin \mathbb{N},\end{cases} \\
l_{2}= \begin{cases}\sigma-\frac{3}{p} & \text { if } \sigma+1-\frac{3}{p} \in \mathbb{N} \\
{\left[\sigma+1-\frac{3}{p}\right]} & \text { if } \sigma+1-\frac{3}{p}>0 \text { and } \sigma+1-\frac{3}{p} \notin \mathbb{N},\end{cases} \\
l_{3}= \begin{cases}\sigma+1-\frac{3}{p} & \text { if } \sigma+2-\frac{3}{p} \in \mathbb{N}, \\
{\left[\sigma+2-\frac{3}{p}\right]} & \text { if } \sigma+2-\frac{3}{p}>0 \text { and } \sigma+2-\frac{3}{p} \notin \mathbb{N},\end{cases}
\end{gathered}
$$

and $[l]$ denotes the integer part of $l$.

Now, we formulate the main result of the paper.

TheOREM 1.1. Let $\sigma \in \mathbb{R}_{+}, p, q \in(1, \infty), 0<T<\infty, \Omega \subset \mathbb{R}^{3}$ be a bounded domain. Assume that $f \in B_{p, q}^{\frac{\sigma}{2}, \sigma}\left(\Omega^{T}\right), b_{k} \in B_{p, q}^{\left(\sigma+1-\frac{1}{p}\right) / 2, \sigma+1-\frac{1}{p}}\left(S^{T}\right), k=1,2, b_{3} \in$ $B_{p, q}^{\frac{\sigma}{2}+1-\frac{1}{2 p}, \sigma+2-\frac{1}{p}}\left(S^{T}\right), u_{0} \in B_{p, q}^{\sigma+2-\frac{2}{p}}(\Omega), S \in C^{2+\sigma}$. Let Assumption 1.1 hold. Moreover, let in the case of $\sigma>\frac{2}{p}$ Assumption 1.2 be satisfied. If $\frac{3}{p}-1<\sigma \leq \frac{2}{p}$ we assume conditions (1.5)-(1.6), and if $\frac{3}{p}-2<\sigma \leq \frac{3}{p}-1$ we assume only (1.6). Then there exists a unique solution $u \in B_{p, q}^{\frac{\sigma}{2}+1, \sigma+2}\left(\Omega^{T}\right)$ to problem (1.1) such that

$$
\int_{0}^{T} \frac{\left\|\partial_{t}^{m_{4}}\left(u-\tilde{u}_{0}\right)\right\|_{L_{p}\left(\Omega^{t}\right)}^{q}}{t^{1+q \lambda_{4}}} d t<\infty
$$

where $\lambda_{4}=\lambda_{1}$,

$$
m_{4}= \begin{cases}{\left[\frac{\sigma}{2}+1\right]} & \text { if } \frac{\sigma}{2} \text { is noninteger } \\ \frac{\sigma}{2} & \text { if } \frac{\sigma}{2} \in \mathbb{N} \cup\{0\} .\end{cases}
$$


Moreover,

$$
\begin{aligned}
& \|u\|_{B_{p, q}^{\frac{\sigma}{2}+1, \sigma+2}\left(\Omega^{T}\right)}+\int_{0}^{T} \frac{\left\|\partial_{t}^{m_{4}}\left(u-\tilde{u}_{0}\right)\right\|_{L_{p}\left(\Omega^{t}\right)}^{q}}{t^{1+q \lambda_{4}}} d t \\
\leq & c\left[\|f\|_{B_{p, q}^{\frac{\sigma}{2}, \sigma}\left(\Omega^{T}\right)}+\sum_{k=1}^{2}\left\|b_{k}\right\|_{B_{p, q}^{\left(\sigma+1-\frac{1}{p}\right) / 2, \sigma+1-\frac{1}{p}}\left(S^{T}\right)}+\left\|b_{3}\right\|_{B_{p, q}^{\frac{\sigma}{2}+1-\frac{1}{2 p}, \sigma+2-\frac{1}{p}}\left(S^{T}\right)}\right. \\
& +\left\|u_{0}\right\|_{B_{p, q}^{\sigma+2-\frac{2}{p}}(\Omega)}+\left(\int_{0}^{T} \frac{\left\|\partial_{t}^{m_{1}} f_{0}\right\|_{L_{p}\left(\Omega^{t}\right)}^{q}}{t^{1+q \lambda_{1}}} d t\right)^{\frac{1}{q}}+\sum_{k=1}^{2}\left(\int_{0}^{T} \frac{\left\|\left.\partial_{t}^{m_{2}} b_{0 k}\right|_{S}\right\|_{L_{p}\left(S^{t}\right)}^{q}}{t^{1+q \lambda_{2}}} d t\right)^{\frac{1}{q}} \\
& \left.+\left(\int_{0}^{T} \frac{\left\|\left.\partial_{t}^{m_{3}} b_{03}\right|_{S}\right\|_{L_{p}\left(S^{t}\right)}^{q}}{t^{1+q \lambda_{3}}} d t\right)^{\frac{1}{q}}\right] .
\end{aligned}
$$

The existence of an extension $\tilde{u}_{0}$ of $u_{0}$ such that the compatibility conditions of Assumption 1.2 are satisfied, follows from Lemma 2.3. However, Lemma 2.3 does not guarantee the fulfillment of Assumption 1.1. Therefore, we use Lemma 2.5 in virtue of which if $q \geq p$ and $\sigma, p, q$ satisfy some additional conditions, we can estimate the integrals from Assumption 1.1 by the norms of $f, b_{i}(i=1,2,3)$ and $u_{0}$. In the case of $q<p$ we can show that these integrals are finite if we assume that $u_{0} \in B_{p, q}^{\sigma+\frac{2 \varepsilon}{p}+2-\frac{2}{p}}(\Omega)$, where $\varepsilon>0$ is sufficiently small. Therefore, an immediate consequence of Theorem 1.1 and Lemmas 2.3, 2.5 is the following theorem.

Theorem 1.2. Let $\sigma \in \mathbb{R}_{+}, p, q \in(1, \infty), 0<T<\infty, \Omega \subset \mathbb{R}^{3}$ be a bounded domain. Assume that $f \in B_{p, q}^{\frac{\sigma}{2}, \sigma}\left(\Omega^{T}\right), b_{k} \in B_{p, q}^{\left(\sigma+1-\frac{1}{p}\right) / 2, \sigma+1-\frac{1}{p}}\left(S^{T}\right), k=1,2, b_{3} \in$ $B_{p, q}^{\frac{\sigma}{2}+1-\frac{1}{2 p}, \sigma+2-\frac{1}{p}}\left(S^{T}\right), u_{0} \in B_{p, q}^{\sigma+2-\frac{2}{p}}(\Omega), S \in C^{2+\sigma}$.

$1^{\circ}$ Let $q \geq p, \frac{\sigma}{2} \neq \frac{1}{p}+\left[\frac{\sigma}{2}\right], \frac{\sigma}{2}-\left[\frac{\sigma}{2}\right]>\frac{1}{p}-\frac{1}{q}, \frac{\sigma}{2} \neq \frac{3}{2 p}-1+\left[\frac{\sigma}{2}+1-\frac{1}{2 p}\right], \frac{\sigma}{2}>$ $\frac{3}{2 p}-1-\frac{1}{q}+\left[\frac{\sigma}{2}+1-\frac{1}{2 p}\right], \frac{\sigma}{2} \neq \frac{3}{2 p}-\frac{1}{2}+\left[\frac{\sigma}{2}+\frac{1}{2}-\frac{1}{2 p}\right], \frac{\sigma}{2}>\frac{3}{2 p}-\frac{1}{2}-\frac{1}{q}+\left[\frac{\sigma}{2}+\frac{1}{2}-\frac{1}{2 p}\right]$. Let in the case of $\sigma>\frac{2}{p}$ Assumption 1.2 be satisfied. If $\frac{3}{p}-1<\sigma \leq \frac{2}{p}$ we assume conditions (1.5)-(1.6), and if $\frac{3}{p}-2<\sigma \leq \frac{3}{p}-1$ we assume only (1.6). Then there exists a unique solution $u \in B_{p, q}^{\frac{\sigma}{2}+1, \sigma+2}\left(\Omega^{T}\right)$ of problem (1.1) and

$$
\begin{gathered}
\|u\|_{B_{p, q}^{\frac{\sigma}{2}+1, \sigma+2}\left(\Omega^{T}\right)} \leq c\left(\|f\|_{B_{p, q}^{\frac{\sigma}{2}, \sigma}\left(\Omega^{T}\right)}+\sum_{k=1}^{2}\left\|b_{k}\right\|_{B_{p, q}^{\left(\sigma+1-\frac{1}{p}\right) / 2, \sigma+1-\frac{1}{p}}\left(S^{T}\right)}\right. \\
\left.+\left\|b_{3}\right\|_{B_{p, q}^{\frac{\sigma}{2}+1-\frac{1}{2 p}, \sigma+2-\frac{1}{p}}\left(S^{T}\right)}+\left\|u_{0}\right\|_{B_{p, q}^{\sigma+2-\frac{2}{p}}(\Omega)}\right)
\end{gathered}
$$

$2^{\circ}$ Let $q<p$ and $\varepsilon>0$ be so small that $\frac{\sigma}{2}-\left[\frac{\sigma}{2}\right]<1-\frac{\varepsilon}{p}, \frac{\sigma}{2}+1-\frac{1}{2 p}-\left[\frac{\sigma}{2}+1-\right.$ $\left.\frac{1}{2 p}\right]<1-\frac{\varepsilon}{p}$ and $\frac{\sigma}{2}+\frac{1}{2}-\frac{1}{2 p}-\left[\frac{\sigma}{2}+\frac{1}{2}-\frac{1}{2 p}\right]<1-\frac{\varepsilon}{p}$. Let $\frac{\sigma}{2} \neq\left[\frac{\sigma}{2}\right]+\frac{1}{q}-\frac{\varepsilon}{p}$, $\frac{\sigma}{2} \neq\left[\frac{\sigma}{2}+1-\frac{1}{2 p}\right]+\frac{1}{2 p}-1+\frac{1}{q}-\frac{\varepsilon}{p}, \frac{\sigma}{2} \neq\left[\frac{\sigma}{2}+\frac{1}{2}-\frac{1}{2 p}\right]+\frac{1}{2 p}-\frac{1}{2}+\frac{1}{q}-\frac{\varepsilon}{p}$.

Moreover, assume that $f \in B_{p, q}^{\frac{\sigma}{2}+\frac{\varepsilon}{p}, \sigma+\frac{2 \varepsilon}{p}}\left(\Omega^{T}\right), b_{k} \in B_{p, q}^{\left(\sigma+1+\frac{\varepsilon}{p}-\frac{1}{p}\right) / 2, \sigma+1+\frac{2 \varepsilon}{p}-\frac{1}{p}}\left(S^{T}\right)$, $k=1,2, b_{3} \in B_{p, q}^{\frac{\sigma}{2}+\frac{\varepsilon}{p}+1-\frac{1}{2 p}, \sigma+\frac{2 \varepsilon}{p}+2-\frac{1}{p}}\left(S^{T}\right), u_{0} \in B_{p, q}^{\sigma+\frac{2 \varepsilon}{p}+2-\frac{2}{p}}(\Omega)$ and in the case of $\frac{\sigma}{2}+\frac{\varepsilon}{p}>\frac{1}{p}$ assume the conditions of Assumption 1.2 with $l_{i}(i=1,2,3)$ replaced 
by

$$
\begin{gathered}
l_{1}^{\prime}= \begin{cases}\sigma+\frac{2 \varepsilon}{p}-\frac{2}{p}-1 & \text { if } \sigma+\frac{2 \varepsilon}{p}-\frac{2}{p} \in \mathbb{N}, \\
{\left[\sigma+\frac{2 \varepsilon}{p}-\frac{2}{p}\right]} & \text { if } \sigma+\frac{2 \varepsilon}{p}-\frac{2}{p}>0 \text { and } \sigma+\frac{2 \varepsilon}{p}-\frac{2}{p} \notin \mathbb{N},\end{cases} \\
l_{2}^{\prime}= \begin{cases}\sigma+\frac{2 \varepsilon}{p}-\frac{3}{p} & \text { if } \sigma+1+\frac{2 \varepsilon}{p}-\frac{3}{p} \in \mathbb{N}, \\
{\left[\sigma+\frac{2 \varepsilon}{p}+1-\frac{3}{p}\right]} & \text { if } \sigma+1+\frac{2 \varepsilon}{p}-\frac{3}{p}>0 \text { and } \sigma+1+\frac{2 \varepsilon}{p}-\frac{3}{p} \notin \mathbb{N}\end{cases}
\end{gathered}
$$

and

$$
l_{3}^{\prime}= \begin{cases}\sigma+\frac{2 \varepsilon}{p}+1-\frac{3}{p} & \text { if } \sigma+\frac{2 \varepsilon}{p}+2-\frac{3}{p} \in \mathbb{N}, \\ {\left[\sigma+\frac{2 \varepsilon}{p}+2-\frac{3}{p}\right]} & \text { if } \sigma+\frac{2 \varepsilon}{p}+2-\frac{3}{p}>0 \text { and } \sigma+\frac{2 \varepsilon}{p}+2-\frac{3}{p} \notin \mathbb{N},\end{cases}
$$

respectively. If $\frac{3}{p}-1<\sigma+\frac{2 \varepsilon}{p} \leq \frac{2}{p}$ we assume conditions (1.5)-(1.6) with $l_{2}$ and $l_{3}$ replaced by $l_{2}^{\prime}$ and $l_{3}^{\prime}$, and if $\frac{3}{p}-2<\sigma<\sigma+\frac{2 \varepsilon}{p} \leq \frac{3}{p}-1$ we assume only (1.6) with $l_{3}$ replaced by $l_{3}^{\prime}$. Then there exists a unique solution $u \in B_{p, q}^{\frac{\sigma}{2}+1, \sigma+2}\left(\Omega^{T}\right)$ with $\int_{0}^{T} \frac{\left\|\partial_{t}^{m_{4}} u\right\|_{L_{p}\left(\Omega^{t}\right)}^{q}}{t^{1+q \lambda_{4}}} d t<\infty$ of problem (1.1) and

$$
\begin{aligned}
& \|u\|_{B_{p, q}^{\frac{\sigma}{2}+1, \sigma+2}\left(\Omega^{T}\right)}+\left(\int_{0}^{T} \frac{\left\|\partial_{t}^{m_{4}} u\right\|_{L_{p}\left(\Omega^{t}\right)}^{q}}{t^{1+q \lambda_{4}}} d t\right)^{1 / q} \\
& \leq c\left[\|f\|_{B_{p, q}^{\frac{\sigma}{2}+\frac{\varepsilon}{p}, \sigma+\frac{2 \varepsilon}{p}}\left(\Omega^{T}\right)}+\sum_{k=1}^{2}\left\|b_{k}\right\|_{B_{p, q}^{\left(\sigma+1+\frac{\varepsilon}{p}-\frac{1}{p}\right) / 2, \sigma+1+\frac{\varepsilon}{p}-\frac{1}{p}}\left(S^{T}\right)}\right. \\
& \left.\quad+\left\|b_{3}\right\|_{B_{p, q}^{\frac{\sigma}{2}+1+\frac{\varepsilon}{p}-\frac{1}{2 p}, \sigma+2+\frac{2 \varepsilon}{p}-\frac{1}{p}}\left(S^{T}\right)}+\left\|u_{0}\right\|_{B_{p, q}^{\sigma+2+\frac{2 \varepsilon}{p}-\frac{2}{p}}(\Omega)}\right],
\end{aligned}
$$

where $m_{4}, \lambda_{4}$ are defined in Theorem 1.1.

In order to prove Theorem 1.1 we consider several auxiliary problems. First, in Section 3 we examine problem (1.1) with $f=0, u_{0}=0$ in the halfspace $\mathbb{R}_{+}^{3}=\left\{x \in \mathbb{R}^{3}\right.$ : $\left.x_{3}>0\right\}$. The main result of Section 3 is formulated in Theorem 3.4. Namely, assuming that $b_{k} \in B_{p, q, \gamma}^{\left(\sigma-1-\frac{1}{p}\right) / 2, \sigma-1-\frac{1}{p}}\left(\mathbb{R}_{+} \times \mathbb{R}^{2}\right), k=1,2$ and $b_{3} \in B_{p, q, \gamma}^{\left(\sigma-\frac{1}{p}\right) / 2, \sigma-\frac{1}{p}}\left(\mathbb{R}_{+} \times \mathbb{R}^{2}\right)$ (see Definition 2.5) we prove the existence of a unique solution $u \in B_{p, q, \gamma}^{\frac{\sigma}{2}, \sigma}\left(\mathbb{R}_{+} \times \mathbb{R}_{+}^{3}\right)$ of the considered problem. To prove Theorem 3.4 we transform problem (1.1) with $f=0$, $u_{0}=0$ to a system of ordinary differential equations with respect to $x_{3}$ by applying the Fourier transform with respect to $x^{\prime}=\left(x_{1}, x_{2}\right)$ and the Laplace transform with respect to $t$ (see (3.2)). We estimate a solution of the system in Besov spaces using the definition by the Fourier-Laplace transform and the Paley-Littlewood decomposition in the directions $x^{\prime}, t$ and the classical one by differences with respect to $x_{3}$ (see Def. 2.4). Using the above existence and appropriate estimate we prove the existence of solutions to problem (1.1) with $u_{0}=0$ in a bounded domain by applying the regularizer technique (see Section 5). Finally, in Section 6 we prove the existence of solutions to problem (1.1) by an appropriate extension of the initial data and using the result from Section 5 .

The crucial point of the paper is the proof of Theorem 3.4 which relies on estimating solution (3.3) of the ordinary differential system (3.2) directly in Besov spaces defined by means of the dyadic decomposition of a partition of unity. The proof bases on delicate scaling arguments connected with the decomposition and estimates of compositions 
of the Fourier and the inverse Fourier transforms. In fact, we prove Theorem 3.4 by a modification and adaptation to the anisotropic case same ideas of Triebel (see [23], Sect. 2.3.6).

In contrast to our existence theorem most of known existence results for linear parabolic or nonstationary Stokes equations have been proved by using either the technique of potentials or the resolvent estimates or the interpolation. Let us recall some of them.

Solonnikov [20] considered initial-boundary value problems for general linear parabolic systems with variable coefficients. He examined the solvability of these problems in anisotropic Hölder and Sobolev spaces $\left.W_{p}^{\sigma, 2 \sigma}((0, T) \times \Omega)\right)$ with $\sigma \in \mathbb{N} \cup\{0\}$.

In [19] Solonnikov obtained estimates for solutions of the heat-equation with appropriate initial and boundary conditions in Besov spaces $B_{p}^{\frac{\sigma}{2}, \sigma}\left(\Omega^{T}\right), \sigma>2$. The results of [19] and [20] are shown by the potential technique.

The methods presented in $[1,21]$ give the existence in spaces $H^{\frac{\sigma}{2}, \sigma}, \sigma \in \mathbb{R}_{+}$. Using the existence of solutions in $W_{p}^{\sigma, 2 \sigma}, \sigma \in \mathbb{N}, p \in(1, \infty)$ (see [20]) and applying the interpolation (see [22]), the existence both in $H_{p}^{\frac{\sigma}{2}, \sigma}$ (Bessel potential spaces) and in $B_{p}^{\frac{\sigma}{2}, \sigma}$ (Besov spaces), $\sigma \in \mathbb{R}_{+}$, can be proved. The existence of solutions in Besov and SobolevSlobodetskii spaces is proved in [2]. The above interpolation technique is applied only in a half space and for solutions with vanishing initial data. The existence of solutions in a bounded domain follows from the regularizer technique.

In $[8,9]$ some existence and uniqueness results in Besov spaces for parabolic equations arising from the compressible Navier-Stokes equations are formulated.

Paper [26] is devoted to the existence result in Besov spaces of the Cauchy problem for abstract parabolic equations of higher order in time.

There are some results concerning the solvability of linear parabolic equations in Besov spaces via semigroups. The most general results are obtained by Amann [3-5], who considered in [3] the following problem

$$
\begin{array}{ll}
u_{t}+A u=f & \text { in } \Omega^{T}, \\
B u=0 & \text { on } S^{T}, \\
\left.u\right|_{t=0}=u_{0} & \text { in } \Omega,
\end{array}
$$

where $A=\sum_{|\alpha| \leq m} a_{\alpha}(x) D^{\alpha}$ and $m=2$.

The above Cauchy problem in the case of $m \in \mathbb{N}, m$ even, is examined in [5]. Amann proves the estimate

$$
\left\|(\lambda+A)^{-1}\right\|_{\mathcal{L}\left(B_{p, q}^{s}, B_{p, q}^{s}\right)} \leq \frac{k}{|\lambda|}, \quad \operatorname{Re} \lambda \geq w>0
$$

where $\mathcal{L}(X, Y)$ is the space of all continuous linear maps from $X$ into $Y$, so - $A$ generates an analytic semigroup.

Paper [3] is concerned with the existence of a semigroup in Bessel potential spaces $H_{p}^{\sigma}$, while in [4] the existence of solutions to evolution equations via semigroup theory in general Banach spaces is examined.

The methods of this paper were applied by the authors to obtain the solvability result for the Cauchy-Dirichlet problem for the heat equation (see [27]). 
There are also some existence results for parabolic pseudo-differential initial-boundary value problems. The following problem is considered in $[10,11,13,14]$ :

$$
\begin{gathered}
u_{t}+P_{\Omega} u+G u=f \quad \text { for }(t, x) \in Q=I \times \Omega, \\
T u=\phi \quad \text { for }(t, x) \in S=I \times \Omega, \\
\left.u\right|_{t=0}=u_{0} \quad \text { for } s \in \Omega,
\end{gathered}
$$

where $\Omega$ is a smooth bounded open set in $\mathbb{R}^{n}$ with boundary $\Gamma, I=(a, b), b \leq+\infty ; P$ is a pseudo-differential operator on $\mathbb{R}^{n}$ of order $d \in \mathbb{Z}$ having the transmission property (see [10]), and $P_{\Omega}$ is its restriction to $\Omega ; G$ is a singular Green operator of order and class $\leq d$. The operators act in $C^{\infty}$ vector bundle $E$ over $\bar{\Omega}$.

Moreover, $T=\left\{T_{0}, \ldots, T_{d-1}\right\}$ is a normal system of trace operators $T_{j}$ of orders $j<d$ and it is assumed that the system $\left\{\partial_{t}+P_{\Omega}+G, T\right\}$ is parabolic.

The above pseudo-differential formulation contains the usual parabolic operator problems as well as the initial-boundary value problems for the Stokes system.

In [11] the solvability of the problem (1.8)-(1.10) in Sobolev-Slobodetskii spaces $H^{\frac{\sigma}{d}, \sigma}$, $\sigma>2$, is proved, while paper [13] gives the existence results in Bessel-potential $H_{p}^{\frac{\sigma}{d}, \sigma}$ and Besov $B_{p}^{\frac{\sigma}{d}, \sigma}$ spaces.

The proofs of the above results base on the study of parameter-dependent elliptic pseudo-differential problems which yield appropriate resolvent estimates (see also $[10,12])$. The next step of these proofs relies either on using the Laplace transform method described by Lions, Magenes [16], Agranovič, Višik [1] and Solonnikov [21] (in the case of paper [11]) or on proving some mapping properties for pseudo-differential operators defined by means of compositions of the inverse Laplace transforms, resolvent opertors and Laplace transforms (see [13]).

Finally, the results of [11] are applied in [14] to study the solvability of different boundary-value problems including Dirichlet, Neumann and intermediate problems for the Navier-Stokes equations in spaces $H^{\frac{\sigma}{2}+1, \sigma+2}(Q)$ for $\sigma+2 \geq \frac{n}{2}, \sigma \geq 0$.

In $[24,25]$ the Besov and Lizorkin-Triebel spaces are used to show the existence of solutions to initial-boundary value problem for the heat equation in $W_{p, q}^{2,1}(\Omega \times(0, T))$.

2. Notation and preliminaries. Let $x=\left(x_{1}, x_{2}, x_{3}\right) \in \mathbb{R}^{3}$. Throughout the paper we use the following notation: $x^{\prime}=\left(x_{1}, x_{2}\right), \bar{x}=\left(x_{0}, x^{\prime}\right)=\left(x_{0}, x_{1}, x_{2}\right)$.

For a derivative with respect to $x^{\prime}$ we use the notation: $\partial_{x^{\prime}}^{k^{\prime}}=\partial_{x_{1}}^{k_{1}} \partial_{x_{2}}^{k_{2}}$, where $k^{\prime}=$ $k_{1}+k_{2} \in \mathbb{N} \cup\{0\}$.

Definition 2.1. Let $\bar{a}=\left(a_{0}, a_{1}, a_{2}\right)=(2,1,1) \equiv a \in \mathbb{R}^{3}$. For $\bar{x} \in \mathbb{R}^{3}$ we introduce the anisotropic distance from zero:

$$
|\bar{x}|_{a}=\left(\sum_{i=0}^{2}\left|x_{i}\right|^{\frac{2}{a_{i}}}\right)^{1 / 2}=\left(\left|x_{0}\right|+\sum_{i=1}^{2}\left|x_{i}\right|^{2}\right)^{1 / 2} .
$$

Let $\mathcal{S}=\mathcal{S}\left(\mathbb{R}^{3}\right)$ be the Schwartz space of all complex-valued rapidly decreasing infinitely differentiable functions on $\mathbb{R}^{3}$. 
Definition 2.2. By $\Phi_{a}\left(\mathbb{R}^{3}\right)$ we denote the collection of all systems $\phi=\left\{\phi_{k}(\bar{x})\right\}_{k=1}^{\infty} \subset$ $\mathcal{S}\left(\mathbb{R}^{3}\right)$ with the properties:

$1^{\circ} \operatorname{supp} \phi_{0} \subset\left\{\bar{x}:|\bar{x}|_{a} \leq 2\right\} ;$

$2^{\circ} \operatorname{supp} \phi_{k} \subset\left\{\bar{x}: 2^{k-1} \leq|\bar{x}|_{a} \leq 2^{k+1}\right\}, k=1,2, \ldots$

$3^{\circ}$ for every multi-index $\bar{\alpha}=\left(\alpha_{0}, \alpha_{1}, \alpha_{2}\right)$ there exists a positive number $c_{\bar{\alpha}}$ such that $2^{k\left(2 \alpha_{0}+\alpha_{1}+\alpha_{2}\right)}\left|D^{\bar{\alpha}} \phi_{k}(\bar{x})\right| \leq c_{\bar{\alpha}}$

for all $k \in \mathbb{N} \cup\{0\}$ and all $\bar{x} \in \mathbb{R}^{3}$;

$4^{\circ} \sum_{k=0}^{\infty} \phi_{k}(\bar{x})=1$ for all $\bar{x} \in \mathbb{R}^{3}$.

In the sequel we use the anisotropic Besov spaces $B_{p, q}^{\frac{\sigma}{2}, \sigma}\left(\mathbb{R} \times \mathbb{R}^{2}\right)$ and $B_{p, q}^{\frac{\sigma}{2}, \sigma}\left(\mathbb{R} \times \mathbb{R}_{+}^{3}\right)$. Definition 2.3 (see $[22,23]$ ). Let $p, q \in(1, \infty), \sigma \in \mathbb{R}_{+}$. The anisotropic Besov space $B_{p, q}^{\frac{\sigma}{2}, \sigma}\left(\mathbb{R} \times \mathbb{R}^{2}\right)$ is the space of functions $u=u\left(t, x^{\prime}\right)$ with the finite norm

$$
\|u\|_{B_{p, q}^{\frac{\sigma}{2}, \sigma}\left(\mathbb{R} \times \mathbb{R}^{2}\right)}=\left[\sum_{k=0}^{\infty}\left(\int_{\mathbb{R} \times \mathbb{R}^{2}}\left|2^{\sigma k}\left(F^{-1} \phi_{k} F u\right)\left(t, x^{\prime}\right)\right|^{p} d t d x^{\prime}\right)^{\frac{q}{p}}\right]^{\frac{1}{q}},
$$

where $\left(F^{-1} \phi_{k} F u\right)\left(t, x^{\prime}\right)=\left(F^{-1}\left(\phi_{k} F u\right)\right)\left(t, x^{\prime}\right)$,

$$
\begin{gathered}
(F u)\left(\xi_{0}, \xi^{\prime}\right)=\int_{\mathbb{R}_{\mathbb{R}^{2}}} e^{-i\left(t \xi_{0}+x^{\prime} \cdot \xi^{\prime}\right)} u\left(t, x^{\prime}\right) d t d x^{\prime}=\int_{\mathbb{R}^{3}} e^{-i \bar{x} \cdot \bar{\xi}} u(\bar{x}) d \bar{x}, \\
\bar{x}=\left(t, x^{\prime}\right)=\left(x_{0}, x^{\prime}\right), \quad \bar{x} \cdot \bar{\xi}=\sum_{i=0}^{2} x_{i} \xi_{i}, \quad \phi=\left\{\phi_{k}(\bar{\xi})\right\}_{k=1}^{\infty} \in \Phi_{a}\left(\mathbb{R}^{3}\right) .
\end{gathered}
$$

Let $\mathbb{R}_{+}^{3}=\left\{x \in \mathbb{R}^{3}: x_{3}>0\right\}$.

Definition 2.4. Let $p, q \in(1, \infty), \sigma \in \mathbb{R}_{+}$. The anisotropic space $B_{p, q}^{\frac{\sigma}{2}, \sigma}\left(\mathbb{R} \times \mathbb{R}_{+}^{3}\right)$ is the space of functions $u=u(t, x)$ with the finite norm

$$
\begin{aligned}
& \|u\|_{B_{p, q}^{\frac{\sigma}{2}, \sigma}\left(\mathbb{R} \times \mathbb{R}_{+}^{3}\right)}=\|u\|_{L_{p}\left(\mathbb{R} \times \mathbb{R}_{+}^{3}\right)} \\
& +\left[\sum_{k=0}^{\infty}\left(\sum_{j \leq[\sigma]} \int_{\mathbb{R}_{\mathbb{R}_{+}^{3}}}\left|2^{(\sigma-j) k}\left(F_{t, x^{\prime}}^{-1} \phi_{k} F_{t, x^{\prime}} \partial_{x_{3}}^{j} u\right)(t, x)\right|^{p} d t d x\right)^{\frac{q}{p}}\right]^{1 / q} \\
& +\left[\sum_{k=0}^{\infty}\left(\int_{\mathbb{R}} d t \int_{\mathbb{R}_{+}} d x_{3} \int_{\mathbb{R}^{\prime}} d z \int_{\mathbb{R}^{2}} d x^{\prime} \frac{\left|F_{t, x^{\prime}}^{-1} \phi_{k} F_{t, x^{\prime}}\left(\partial_{x_{3}}^{[\sigma]} u\left(\bar{x}, x_{3}+z\right)-\partial_{x_{3}}^{[\sigma]} u\left(\bar{x}, x_{3}\right)\right)\right|^{p}}{|z|^{1+p(\sigma-[\sigma])}}\right)^{\frac{q}{p}}\right]^{\frac{1}{q}},
\end{aligned}
$$

where

$$
\begin{aligned}
& \left(F_{t, x^{\prime}}^{-1} \phi_{k} F_{t, x^{\prime}} \partial_{x_{3}}^{j} u\right)(t, x)=\left(F_{t, x^{\prime}}^{-1}\left(\phi_{k} F_{t, x^{\prime}} \partial_{x_{3}}^{j} u\right)\right)(t, x) \\
& \left(F_{t, x^{\prime}} u\right)\left(\bar{\xi}, x_{3}\right)=\int_{\mathbb{R} \times \mathbb{R}^{2}} e^{-i\left(t \xi_{0}+x^{\prime} \xi^{\prime}\right)} u(t, x) d t d x^{\prime}=\int_{\mathbb{R} \times \mathbb{R}^{2}} e^{-i \bar{x} \cdot \bar{\xi}} u\left(\bar{x}, x_{3}\right) d \bar{x} .
\end{aligned}
$$

Let $u=u\left(t, x^{\prime}\right)$ or $u=u(t, x)$ be a function vanishing for $t<0$. We define the function

$$
u_{\gamma}= \begin{cases}e^{-\gamma t} u & \text { for } t>0 \\ 0 & \text { for } t<0\end{cases}
$$


Using $u_{\gamma}$ we can introduce spaces $B_{p, q, \gamma}^{\frac{\sigma}{2}, \sigma}\left(\mathbb{R}_{+} \times Q\right)$ (where $Q=\mathbb{R}^{2}$ or $Q=\mathbb{R}_{+}^{3}$ ) of functions $u=u\left(t, x^{\prime}\right)$ or $u=u(t, x)$ as follows.

\section{Definition 2.5.}

$$
B_{p, q, \gamma}^{\frac{\sigma}{2}, \sigma}\left(\mathbb{R}_{+} \times Q\right)=\left\{u: u_{\gamma} \in B_{p, q}^{\frac{\sigma}{2}, \sigma}(\mathbb{R} \times Q)\right\}, \quad\|u\|_{B_{p, q, \gamma}^{\frac{\sigma}{2}, \sigma}\left(\mathbb{R}_{+} \times Q\right)}=\left\|u_{\gamma}\right\|_{B_{p, q}^{\frac{\sigma}{2}, \sigma}(\mathbb{R} \times Q)} .
$$

Since $F^{-1}\left(\phi_{k}\left(F u_{\gamma}\right)\right)=e^{-\gamma t} F_{1}^{-1}\left(\phi_{k} F_{1} u\right), F_{t, x^{\prime}}^{-1}\left(\phi_{k} F_{t, x^{\prime}} u_{\gamma}\right)=e^{-\gamma t} F_{2}^{-1}\left(\phi_{k} F_{2}\right)$, for a sufficiently regular and rapidly decreasing function $u$ which vanishes for $t<0$, where $F_{1}, F_{2}$ are the Fourier-Laplace transforms, i.e. for $s=\gamma+i \xi_{0}, \operatorname{Re} s=\gamma>0$,

$$
\begin{aligned}
\left(F_{1} u\right)\left(s, \xi^{\prime}\right) & =\int_{\mathbb{R}_{+}} e^{-s t} d t \int_{\mathbb{R}^{2}} e^{-i x^{\prime} \cdot \xi^{\prime}} u\left(t, x^{\prime}\right) d x^{\prime}, \\
\left(F_{2} u\right)\left(s, \xi^{\prime}, x_{3}\right) & =\int_{\mathbb{R}_{+}} e^{-s t} d t \int_{\mathbb{R}^{2}} e^{-i x^{\prime} \cdot \xi^{\prime}} u(t, x) d x^{\prime},
\end{aligned}
$$

the norm $\|u\|_{B_{p, q, \gamma}^{\frac{\sigma}{2}, \sigma}\left(\mathbb{R}_{+} \times Q\right)}$ can be written in the following way:

$$
\|u\|_{B_{p, q, \gamma}^{\frac{\sigma}{2}, \sigma}\left(\mathbb{R}_{+} \times \mathbb{R}^{2}\right)}=\left\|u_{\gamma}\right\|_{L_{p}\left(\mathbb{R}_{+} \times \mathbb{R}^{2}\right)}+\left[\sum_{k=0}^{\infty}\left(\int_{\mathbb{R}_{+} \times \mathbb{R}^{2}}\left|e^{-\gamma t} 2^{\sigma k}\left(F_{1}^{-1} \phi_{k} F_{1} u\right)\left(t, x^{\prime}\right)\right|^{p} d t d x^{\prime}\right)^{\frac{q}{p}}\right]^{\frac{1}{q}}
$$

if $Q=\mathbb{R}^{2}$ or

$$
\begin{aligned}
& \|u\|_{B_{p, q, \gamma}^{\frac{\sigma}{2}, \sigma}\left(\mathbb{R}_{+} \times \mathbb{R}_{+}^{3}\right)}=\left\|u_{\gamma}\right\|_{L_{p}\left(\mathbb{R}_{+} \times \mathbb{R}_{+}^{3}\right)} \\
& +\left[\sum_{k=0}^{\infty}\left(\sum_{j \leq[\sigma]} \int_{\mathbb{R} \times \mathbb{R}_{+}^{3}}\left|e^{-\gamma t} 2^{(\sigma-j) k}\left(F_{2}^{-1} \phi_{k} F_{2} u\right)(t, x)\right|^{p} d t d x\right)^{\frac{q}{p}}\right]^{\frac{1}{q}} \\
& +\left[\sum_{k=0}^{\infty}\left(\int_{\mathbb{R}^{2}} d t \int_{\mathbb{R}_{+}} d x_{3} \int_{\mathbb{R}} d z \int_{\mathbb{R}^{2}} d x^{\prime} \frac{\left.\left|e^{-\gamma t} F_{2}^{-1} \phi_{k} F_{2}\left(\partial_{x_{3}}^{[\sigma]} u\left(\bar{x}, x_{3}+z\right)-\partial_{x_{3}}^{[\sigma]} u\left(\bar{x}, x_{3}\right)\right)\right|^{p}\right)}{|z|^{1+p(\sigma-[\sigma])}}\right)^{\frac{q}{p}}\right]^{\frac{1}{q}} \\
& =\left\|u_{\gamma}\right\|_{L_{p}\left(\mathbb{R}_{+} \times \mathbb{R}_{+}^{3}\right)}+\|u\|_{1, B_{p, q, \gamma}^{\frac{\sigma}{2}, \sigma}\left(\mathbb{R}_{+} \times \mathbb{R}_{+}^{3}\right)}+\|u\|_{2, B_{p, q, \gamma}^{\frac{\sigma}{2}, \sigma}\left(\mathbb{R}_{+} \times \mathbb{R}_{+}^{3}\right)}
\end{aligned}
$$

if $Q=\mathbb{R}_{+}^{3}$.

Now, we shall introduce definitions of Besov spaces $B_{p, q}^{\sigma}(\Omega)$ and $B_{p, q}^{\frac{\sigma}{2}, \sigma}\left(\Omega^{T}\right)$, where $\Omega \subset \mathbb{R}^{n}$ is an open domain. In the special cases of $\Omega=\mathbb{R} \times \mathbb{R}^{2}$ or $\Omega=\mathbb{R} \times \mathbb{R}_{+}^{3}$ these definitions are equivalent to Definitions 2.3 and 2.4 , respectively.

Let $\sigma \in \mathbb{R}_{+}, p, q \in(1, \infty)$ and let

$$
\begin{aligned}
\Delta_{i}^{m}(h) u(x) & =\sum_{j=0}^{m}(-1)^{m-j}\left(\begin{array}{c}
m \\
j
\end{array}\right) u\left(x+j h e_{i}\right), \\
\Delta_{i}^{m}(h, \Omega) u(x) & = \begin{cases}\Delta_{i}^{m}(h) u(x) & \text { if }\left[x, x+h m e_{i}\right] \subset \Omega, \\
0 & \text { otherwise, }\end{cases}
\end{aligned}
$$

where $x=\left(x_{1}, \ldots, x_{n}\right), m \in \mathbb{N}, e_{i}$ is the unit vector directed along the $x_{i}$ axis. 
Definition 2.6 (see $[6,17]$ ). The Besov space $B_{p, q}^{\sigma}(\Omega)$ is the space of functions $u=u(x)$ with the finite norm

$$
\|u\|_{B_{p, q}^{\sigma}(\Omega)}=\|u\|_{L_{p}(\Omega)}+\sum_{i=1}^{n}\left(\int_{0}^{h_{0}} \frac{\left\|\Delta_{i}^{m}(h, \Omega) \partial_{x_{i}}^{k} u\right\|_{L_{p}(\Omega)}^{q}}{h^{1+q(\sigma-k)}} d h\right)^{\frac{1}{q}}
$$

where $h_{0}>0, m>\sigma-k>0, k \in \mathbb{N} \cup\{0\}, \sigma \in \mathbb{R}_{+}$.

Now, for $m_{0} \in \mathbb{N}$ let

$$
\begin{aligned}
\Delta_{t}^{m_{0}}(h) u(t) & =\sum_{j=0}^{m_{0}}(-1)^{m_{0}-j}\left(\begin{array}{c}
m_{0} \\
j
\end{array}\right) u(t+j h), \\
\Delta_{i}^{m_{0}}(h,(\tau, T)) u(t) & = \begin{cases}\Delta_{i}^{m_{0}}(h) u(t) & \text { if }\left[t, t+m_{0} h\right] \subset(\tau, T), \\
0 & \text { otherwise. }\end{cases}
\end{aligned}
$$

Definition 2.7 (see $[6,17])$. The Besov space $B_{p, q}^{\frac{\sigma}{2}, \sigma}((\tau, T) \times \Omega)(-\infty<\tau<T<\infty)$ is the space of functions $u=u(t, x)$ with the finite norm

$$
\begin{aligned}
\|u\|_{B_{p, q}^{\frac{\sigma}{2}, \sigma}((\tau, T) \times \Omega)}= & \|u\|_{L_{p}((\tau, T) \times \Omega)} \\
& +\sum_{i=1}^{n}\left(\int_{0}^{h_{0}} \frac{\left\|\Delta_{i}^{m}(h, \Omega) \partial_{x_{i}}^{k} u\right\|_{L_{p}((\tau, T) \times \Omega)}^{q}}{h^{1+q(\sigma-k)}} d h\right)^{\frac{1}{q}} \\
& +\left(\int_{0}^{h_{0}} \frac{\left\|\Delta_{t}^{m_{0}}(h,(\tau, T)) \partial_{t}^{k_{0}} u\right\|_{L_{p}((\tau, T) \times \Omega)}^{q}}{h^{1+q\left(\sigma / 2-k_{0}\right)}} d h\right)^{\frac{1}{q}},
\end{aligned}
$$

where $h_{0}>0, m>\sigma-k, m_{0}>\frac{\sigma}{2}-k_{0}, k_{0} \in \mathbb{N} \cup\{0\}, \sigma \in \mathbb{R}_{+}, \sigma>k, \frac{\sigma}{2}>k_{0}$.

The Besov space $B_{p, q}^{\frac{\sigma}{2}, \sigma}((\tau, T) \times S)$, where $S$ is the boundary of a bounded domain $\Omega \subset \mathbb{R}^{n}$ is defined in a standard way by using local coordinates and a partition of unity.

The following lemma holds.

LEMMA 2.1. In the case of $\Omega=\mathbb{R}^{3}$ and $(\tau, T)=(-\infty,+\infty)$ the norms in $B_{p, q}^{\frac{\sigma}{2}, \sigma}((\tau, T) \times$ $\Omega$ ) introduced in Definitions 2.3 and 2.7 are equivalent.

Similarly, in the case of $(\tau, T)=(-\infty,+\infty)$ and $\Omega=\mathbb{R}_{+}^{3}$ the norms introduced in Definitions 2.4 and 2.7 are equivalent.

Now, we define a collection $\mathcal{A}_{a L}\left(\mathbb{R}^{3}\right)$ containing the system $\phi=\left\{\phi_{j}(\bar{x})\right\}_{j=0}^{\infty} \in \Phi_{a}\left(\mathbb{R}^{3}\right)$. In Section 3 we use the properties of this collection.

Definition 2.8 (see [23]). Let $L>0$ be a given natural number. By $\mathcal{A}_{a L}\left(\mathbb{R}^{3}\right)$ we denote the collection of systems $\phi=\left\{\phi_{j}(\bar{x})\right\}_{j=0}^{\infty} \subset \mathcal{S}\left(\mathbb{R}^{3}\right)$ of functions with compact supports such that

$$
\begin{aligned}
C(\phi)= & \sup _{\bar{x} \in \mathbb{R}^{3}}|\bar{x}|_{a}^{2} \sum_{|\bar{\alpha}| \leq L}\left|D_{\bar{x}}^{\bar{\alpha}} \phi_{0}(\bar{x})\right| \\
& +\sup _{\substack{\bar{x} \in \mathbb{R}^{3} \backslash\{0\} \\
j=1,2, \ldots}}\left(|\bar{x}|_{a}^{L}+|\bar{x}|_{a}^{-L}\right) \sum_{|\bar{\alpha}| \leq L}\left|D_{\bar{x}}^{\bar{\alpha}} \phi_{j}\left(2^{2 j} x_{0}, 2^{j} x^{\prime}\right)\right|<\infty,
\end{aligned}
$$

where $D_{\bar{x}}^{\bar{\alpha}}=\partial_{x_{0}}^{\alpha_{0}} \partial_{x_{1}}^{\alpha_{1}} \partial_{x_{2}}^{\alpha_{2}}, \sum_{i=0}^{2} \alpha_{i}=|\bar{\alpha}|$.

To prove Lemma 4.1 we need the extension lemma. 
Lemma 2.2 (see [6], Ch. 4, Sect. 18). Let $\sigma \in \mathbb{R}_{+}, p, q \in(1, \infty), f \in B_{p, q}^{\frac{\sigma}{2}, \sigma}\left(\mathbb{R} \times \mathbb{R}_{+}^{3}\right)$, $R_{+}^{3}=\left\{x \in \mathbb{R}^{3}: x=\left(x_{1}, x_{2}, x_{3}\right), x_{3}>0\right\}$. Then there exists an extension of $f$ onto $\mathbb{R} \times \mathbb{R}^{3}$ denoted by $f^{\prime}$ such that $\left.f^{\prime}\right|_{\mathbb{R} \times \mathbb{R}_{+}^{3}}=f$ and

$$
\left\|f^{\prime}\right\|_{B_{p, q}^{\frac{\sigma}{2}, \sigma}\left(\mathbb{R} \times \mathbb{R}^{3}\right)} \leq c\|f\|_{B_{p, q}^{\frac{\sigma}{2}, \sigma}\left(R \times \mathbb{R}_{+}^{3}\right)},
$$

where $c>0$ does not depend on $f$.

We also use the following trace lemma:

Lemma 2.3 (see [17]). Let $u \in B_{p, q}^{\frac{\sigma}{2}, \sigma}\left(\Omega^{T}\right), \sigma \in \mathbb{R}_{+}, \sigma>\frac{2}{p}, p, q \in(1, \infty)$. Then $\left.u\right|_{t=0}=$ $\phi \in B_{p, q}^{\sigma-\frac{2}{p}}(\Omega)$ and

$$
\|\phi\|_{\substack{\sigma-\frac{2}{p} \\ B_{p, q}(\Omega)}} \leq c\|u\|_{B_{p, q}^{\frac{\sigma}{2} \cdot \sigma}\left(\Omega^{T}\right)} .
$$

Let $\phi^{(k)} \in B_{p, q}^{\sigma-\frac{2}{p}-2 k}(\Omega)$, where $k=0,1, \ldots, l$,

$$
l= \begin{cases}\frac{\sigma}{2}-\frac{1}{p}-1 & \text { if } \frac{\sigma}{2}-\frac{1}{p} \in \mathbb{N} \\ {\left[\frac{\sigma}{2}-\frac{1}{p}\right]} & \text { if } \frac{\sigma}{2}-\frac{1}{p} \notin \mathbb{N} .\end{cases}
$$

Then there exists a function $u \in B_{p, q}^{\frac{\sigma}{2}, \sigma}\left(\Omega^{T}\right)$ such that $\left.\partial_{t}^{k} u\right|_{t=0}=\phi^{(k)}, k=0,1, \ldots, l$ and

$$
\|u\|_{B_{p, q}^{\frac{\sigma}{2}, \sigma}\left(\Omega^{T}\right)} \leq c \sum_{k=0}^{l}\left\|\phi^{(k)}\right\|_{B_{p, q}^{\sigma-\frac{2}{p}-2 k}(\Omega)} .
$$

In order to prove Theorem 1.1 we also need the following lemma.

Lemma 2.4 (see [27], Lemma 2.5). Let $\sigma \in \mathbb{R}_{+}, p, q \in(1, \infty), f \in B_{p}^{\frac{\sigma}{2}, \sigma}\left(\Omega^{T}\right)$ and let

$$
f^{\prime}= \begin{cases}f & \text { for } t \in(0, T) \\ 0 & \text { for } t<0\end{cases}
$$

Then $f^{\prime} \in B_{p, q}^{\frac{\sigma}{2}, \sigma}((-\infty, T) \times \Omega)$ if and only if the following conditions are satisfied:

$$
\int_{0}^{T} \frac{\left\|\partial_{t}^{m} f\right\|_{L_{p}\left(\Omega^{t}\right)}^{q}}{t^{1+q \lambda}} d t<\infty
$$

where

$$
m=\left\{\begin{array}{ll}
{[\sigma / 2]} & \text { if } \sigma / 2 \text { is noninteger, } \\
\frac{\sigma}{2}-1 & \text { if } \frac{\sigma}{2} \in \mathbb{N},
\end{array} \quad \lambda= \begin{cases}\sigma / 2-[\sigma / 2] & \text { if } \sigma / 2 \text { is noninteger }, \\
1 & \text { if } \frac{\sigma}{2} \in \mathbb{N} ;\end{cases}\right.
$$

where

$$
\left.\partial_{t}^{l} f\right|_{t=0}=0
$$

$$
l= \begin{cases}{[\sigma / 2]-1} & \text { if } \frac{\sigma}{2} \text { is noninteger and }\left[\frac{\sigma}{2}\right] \geq 1, \\ \frac{\sigma}{2}-2 & \text { if } \frac{\sigma}{2}-2 \in \mathbb{N} \cup\{0\} .\end{cases}
$$

Moreover, for $f^{\prime} \in B_{p, q}^{\frac{\sigma}{2}, \sigma}((-\infty, T) \times \Omega)$ we have the estimate

$$
\begin{aligned}
& c_{1}\left[\|f\|_{B_{p, q}^{\frac{\sigma}{2}, \sigma}\left(\Omega^{T}\right)}+\left(\int_{0}^{T} \frac{\left\|\partial_{t}^{m} f\right\|_{L_{p}\left(\Omega^{t}\right)}^{q}}{t^{1+q \lambda}} d t\right)^{\frac{1}{q}}\right] \leq\left\|f^{\prime}\right\|_{B_{p, q}^{\frac{\sigma}{2}, \sigma}((-\infty, T) \times \Omega)} \\
& \leq c_{2}\left[\|f\|_{B_{p, q}^{\frac{\sigma}{2}, \sigma}\left(\Omega^{T}\right)}+\left(\int_{0}^{T} \frac{\left\|\partial_{t}^{m} f\right\|_{L_{p}\left(\Omega^{t}\right)}^{q}}{t^{1+q \lambda}} d t\right)^{\frac{1}{q}}\right]
\end{aligned}
$$


REMARK 1. The result similar to Lemma 2.4 can be proved for a function $b \in B_{p, q}^{\frac{\sigma}{2}, \sigma}\left(S^{T}\right)$ extended by zero on $t<0$.

Finally, we present a lemma which in some cases guarantees together with Lemma 2.3 the possibility of constructing an extension $\tilde{u}_{0}$ of $\tilde{u}_{0}$ such that Assumption 1.1 holds.

Lemma 2.5 (see [27], Lemma 2.6). Let $p, q \in(1, \infty), \sigma \in \mathbb{R}_{+}$,

$$
m=\left\{\begin{array}{ll}
{\left[\frac{\sigma}{2}\right]} & \text { if } \frac{\sigma}{2} \text { is noninteger, } \\
\frac{\sigma}{2}-1 & \text { if } \frac{\sigma}{2} \in \mathbb{N},
\end{array} \quad \lambda= \begin{cases}\frac{\sigma}{2}-\left[\frac{\sigma}{2}\right] & \text { if } \frac{\sigma}{2} \text { is noninteger }, \\
1 & \text { if } \frac{\sigma}{2} \in \mathbb{N},\end{cases}\right.
$$

$1^{\circ}$ Let $q \geq p, \frac{\sigma}{2} \neq \frac{1}{p}+\left[\frac{\sigma}{2}\right], \frac{\sigma}{2}>\left[\frac{\sigma}{2}\right]+\frac{1}{p}-\frac{1}{q}, f \in B_{p, q}^{\frac{\sigma}{2}, \sigma}\left(\Omega^{T}\right)$. Moreover, in the case of $\frac{\sigma}{2}>\frac{1}{p}$ assume that

$$
\left.\partial_{t}^{i} f\right|_{t=0}=0 \quad \text { for } 2 i \leq m^{\prime}
$$

where

$$
m^{\prime}= \begin{cases}\sigma-\frac{2}{p}-1 & \text { if } \sigma-\frac{2}{p} \in \mathbb{N}, \\ {\left[\sigma-\frac{2}{p}\right]} & \text { if } \sigma-\frac{2}{p}>0 \text { and } \sigma-\frac{2}{p} \notin \mathbb{N} .\end{cases}
$$

Then

$$
\int_{0}^{T} \frac{\left\|\partial_{t}^{m} f\right\|_{L_{p}\left(\Omega^{t}\right)}^{q}}{t^{1+q \lambda}} d t \leq c\|f\|_{B_{p, q}^{\frac{\sigma}{2}, \sigma}\left(\Omega^{T}\right)}^{q} .
$$

$2^{\circ}$ Let $q<p$ and let $\varepsilon>0$ be so small that $\frac{\sigma}{2}-\left[\frac{\sigma}{2}\right]<1-\frac{\varepsilon}{p}$. Let $\frac{\sigma}{2} \neq\left[\frac{\sigma}{2}\right]+\frac{1}{q}-\frac{\varepsilon}{p}$ and $f \in B_{p, q}^{\frac{\sigma}{2}+\frac{\varepsilon}{p}, \sigma+\frac{2 \varepsilon}{p}}\left(\Omega^{T}\right)$. Moreover, in the case of $\frac{\sigma}{2}+\varepsilon>\frac{1}{p}$ assume that

$$
\left.\partial_{t}^{i} f\right|_{t=0}=0 \quad \text { for } 2 i \leq m^{\prime \prime}
$$

where

$$
m^{\prime \prime}= \begin{cases}\sigma+2 \varepsilon-\frac{2}{p}-1 & \text { if } \sigma+2 \varepsilon-\frac{2}{p} \in \mathbb{N}, \\ {\left[\sigma+2 \varepsilon-\frac{2}{p}\right]} & \text { if } \sigma+2 \varepsilon-\frac{2}{p}>0 \text { and } \sigma+2 \varepsilon-\frac{2}{p} \notin \mathbb{N} .\end{cases}
$$

Then

$$
\int_{0}^{T} \frac{\left\|\partial_{t}^{m} f\right\|_{L_{p}\left(\Omega^{t}\right)}^{q}}{t^{1+q \lambda}} d t \leq c\|f\|_{B_{p, q}^{\frac{\sigma}{2}+\frac{\varepsilon}{p}, \sigma+\frac{2 \varepsilon}{p}}\left(\Omega^{T}\right)} .
$$

To define a regularizer and to prove the existence theorem for problem (1.1), where $\Omega \subset \mathbb{R}^{3}$ is bounded domain, we need a partition of unity. To introduce this we define two families of open sets $\left\{w^{(k)}\right\}$ and $\left\{\Omega^{(k)}\right\}, k \in \mathfrak{M} \cup \mathfrak{N}$, such that $\bar{w}^{(k)} \subset \Omega^{(k)} \subset \Omega$, $\bigcup_{k} w^{(k)}=\bigcup_{k} \Omega^{(k)}=\Omega, \bar{\Omega}^{(k)} \cap S=\phi$ for $k \in \mathfrak{M}$ and $\bar{\Omega}^{(k)} \cap S \neq \phi$ for $k \in \mathfrak{N}$. We assume that at most $N_{0}$ of the $\Omega^{(k)}$ have nonempty intersection and $\sup _{k} \operatorname{diam} \Omega^{(k)} \leq 2 \lambda$, $\sup _{k} \operatorname{diam} w^{(k)} \leq \lambda$ for some $\lambda>0$. Let $\zeta^{(k)}(x)$ be a smooth function with the properties: $0 \leq \zeta^{(k)}(x) \leq 1$ for $x \in \Omega, \zeta^{(k)}(x)=1$ for $x \in w^{(k)}, \zeta^{(k)}(x)=0$ for $x \in \Omega \backslash \Omega^{(k)}$ and $\left|D_{x}^{\alpha} \zeta^{(k)}(x)\right| \leq \frac{c}{\lambda^{|\alpha|}}, \alpha=\left(\alpha_{1}, \alpha_{2}, \alpha_{3}\right)$. Then $1 \leq \sum_{k \in \mathfrak{M} \cup \mathfrak{N}}\left(\zeta^{(k)}(x)\right)^{2} \leq N_{0}$. Introducing the function

$$
\eta^{(k)}(x)=\frac{\zeta^{(k)}(x)}{\sum_{l \in \mathfrak{M} \cup \mathfrak{N}}\left(\zeta^{(l)}(x)\right)^{2}},
$$

we have that $\eta^{(k)}(x)=0$ for $x \in \Omega \backslash \Omega^{(k)}, \sum_{k \in \mathfrak{M} \cup \mathfrak{N}} \eta^{(k)}(x) \zeta^{(k)}(x)=1$ and $\left|D_{x}^{\alpha} \eta^{(k)}(x)\right| \leq$ $c / \lambda^{|\alpha|}$. 
Considering problems invariant with respect to translations and rotations we associate with each $\Omega^{(k)}, k \in \mathfrak{N}$, a local system of coordinates $y=\left(y_{1}, y_{2}, y_{3}\right)$ with center at $\xi^{(k)} \in S^{(k)}=S \cap \bar{\Omega}^{(k)}$ such that $w^{(k)}$ and $\Omega^{(k)}$ are described by the inequalities

$$
\left|y_{i}\right|<\lambda, \quad i=1,2,3, \quad 0<y_{3}-F\left(y_{1}, y_{2}\right)<\lambda
$$

and

$$
\left|y_{i}\right|<2 \lambda, \quad i=1,2,3, \quad 0<y_{3}-F\left(y_{1}, y_{2}\right)<2 \lambda,
$$

respectively. Then $S^{(k)}$ can be written as

$$
S^{(k)}=\left\{y: y_{3}=F\left(y_{1}, y_{2}\right),\left|y_{i}\right|<2 \lambda, i=1,2\right\} .
$$

We straighten the boundary $S^{(k)}$ by introducing the new coordinates

$$
z_{i}=y_{i}, \quad i=1,2, \quad z_{3}=y_{3}-F\left(y_{1}, y_{2}\right) .
$$

We denote the above transformation by $\Phi_{k}$, i.e.

$$
z=\Phi_{k}(y) \quad \text { for } y \in S^{(k)} \text {. }
$$

Then, denote: $\hat{S}^{(k)}=\Phi_{k}\left(S^{(k)}\right), \hat{\Omega}^{(k)}=\Phi_{k}\left(\Omega^{(k)}\right), \hat{w}^{(k)}=\Phi_{k}\left(w^{(k)}\right)$. The sets $\hat{w}^{(k)}$ and $\hat{\Omega}^{(k)}$ are described by the inequalitites:

$$
\begin{aligned}
& \left|z_{i}\right|<\lambda, \quad i=1,2, \quad 0<z_{3}<\lambda, \\
& \left|z_{i}\right|<2 \lambda, \quad i=1,2, \quad 0<z_{3}<2 \lambda,
\end{aligned}
$$

respectively. Let $y=Y_{k}(x)$ be the transformation from the coordinates $x$ to the local coordinates $y$, which is a composition of a translation and a rotation. We set

$$
\hat{u}^{(k)}(t, z)=u\left(t, Y_{k}^{-1} \circ \Phi_{k}^{-1}(z)\right) .
$$

3. The parabolic problem with $f=0, b_{k} \neq 0, u_{0}=0$ in the half space. The aim of this section is to prove the existence of solutions to the problem

$$
\begin{array}{ll}
u_{t}-\operatorname{div} \mathbb{D}(u)=0 & \text { in } \mathbb{R}_{+} \times \mathbb{R}_{+}^{3}, \\
\mu\left(\frac{\partial u_{k}}{\partial x_{3}}+\frac{\partial u_{3}}{\partial x_{k}}\right)=b_{k}, \quad k=1,2, & \text { in } \mathbb{R}_{+} \times \mathbb{R}^{2}, \\
u_{3}=b_{3} & \text { in } \mathbb{R}_{+} \times \mathbb{R}^{2}, \\
\left.u\right|_{t=0}=0 & \text { in } \mathbb{R}_{+}^{3},
\end{array}
$$

where $\mathbb{R}_{+}^{3}=\left\{x \in \mathbb{R}^{3}: x=\left(x_{1}, x_{2}, x_{3}\right), x_{3}>0\right\}$.

In order to solve (3.1) we apply the Fourier-Laplace transform $F_{2}$. Then (3.1) implies

$$
\begin{aligned}
& \mu \frac{d^{2} \hat{u}_{k}}{d x_{3}^{2}}+\nu i \xi_{k} \frac{d \hat{u}_{3}}{d x_{3}}-\left(s+\mu \xi^{\prime 2}\right) \hat{u}_{k}-\nu \xi_{k} \xi_{j} \hat{u}_{j}=0, \quad k=1,2, \quad \text { for } x_{3}>0, \\
& (\mu+\nu) \frac{d^{2} \hat{u}_{3}}{d x_{3}^{2}}+\nu i \xi_{j} \frac{d \hat{u}_{j}}{d x_{3}}-\left(s+\mu \xi^{\prime 2}\right) \hat{u}_{3}=0 \quad \text { for } x_{3}>0, \\
& \mu \frac{d \hat{u}_{k}}{d x_{3}}+\mu i \xi_{k} \hat{u}_{3}=\hat{b}_{k}, \quad k=1,2, \quad \text { for } x_{3}=0, \\
& \hat{u}_{3}=\hat{b}_{3} \\
& \text { for } x_{3}=0 \text {, }
\end{aligned}
$$


where $\xi^{\prime}=\left(\xi_{1}, \xi_{2}\right), \xi^{\prime 2}=\xi_{1}^{2}+\xi_{2}^{2}, s=\gamma+i \xi_{0}, \gamma>0, \hat{u}_{k}=F_{2} u_{k}, k=1,2,3$ and the summation convention over repeated indices is assumed.

We are looking for a solution to (3.2) which vanishes for $x_{3} \rightarrow \infty$. Such a solution to (3.2) vanishing for $x_{3} \rightarrow \infty$ has the form (see [7])

$$
\hat{u}=\Phi(\bar{\xi}) e^{-\tau_{1} x_{3}}+\Psi(\bar{\xi})\left(\xi_{1}, \xi_{2}, i \tau_{2}\right) e^{-\tau_{2} x_{3}},
$$

where $\bar{\xi}=\left(\xi_{0}, \xi^{\prime}\right), \Phi(\bar{\xi})=\left(\Phi_{1}, \Phi_{2}, \frac{i}{\tau_{1}} \xi^{\prime} \cdot \Phi^{\prime}\right), \Phi_{k}=\Phi_{k}(\bar{\xi}), k=1,2, \Phi^{\prime}=\left(\Phi_{1}, \Phi_{2}\right)$, $\tau_{1}=\sqrt{\frac{s}{\mu}+\xi^{\prime 2}}, \tau_{2}=\sqrt{\frac{s}{\mu+\nu}+\xi^{\prime 2}}, \arg \tau_{k} \in\left(-\frac{\pi}{4}, \frac{\pi}{4}\right), k=1,2, \xi^{\prime} \cdot \Phi^{\prime}=\xi_{1} \Phi_{1}+\xi_{2} \Phi_{2}$. Inserting (3.3) into (3.2) yields

$$
\begin{aligned}
& \tau_{1} \Phi_{k}+\tau_{2} \xi_{k} \Psi=i \hat{b}_{3} \xi_{k}-\frac{\hat{b}_{k}}{\mu}, \quad k=1,2, \\
& \xi^{\prime} \cdot \Phi^{\prime}+\tau_{1} \tau_{2} \Psi=-i \tau_{1} \hat{b}_{3} .
\end{aligned}
$$

Solving (3.4) we obtain (see [7])

$$
\begin{aligned}
& \Phi_{k}=-\frac{1}{s \tau_{1}} \hat{b}^{\prime} \cdot \xi^{\prime} \xi_{k}-\frac{1}{\mu \tau_{1}} \hat{b}_{k}, \quad k=1,2, \\
& \Psi=\frac{1}{\tau_{2}}\left(i \hat{b}_{3}+\frac{1}{s} \hat{b}^{\prime} \cdot \xi^{\prime}\right) .
\end{aligned}
$$

Let

$$
e_{i}=e^{-\tau_{i} x_{3}}, \quad i=1,2, \quad e_{0}=\frac{e_{1}-e_{2}}{\tau_{1}-\tau_{2}}
$$

Then (3.3) can be written as

$$
\hat{u}=V e_{0}+W e_{1},
$$

where $V=\left(V_{1}, V_{2}, V_{3}\right), W=\left(W_{1}, W_{2}, W_{3}\right)$, and

$$
\begin{aligned}
V_{k} & =\frac{1}{\tau_{2}}\left(i \hat{b}_{3}+\frac{1}{s} \hat{b}^{\prime} \cdot \xi^{\prime}\right)\left(\tau_{2}-\tau_{1}\right) \xi_{k}=\frac{-c_{0}}{\tau_{2}\left(\tau_{1}+\tau_{2}\right)}\left(i \hat{b}_{3} s+\hat{b}^{\prime} \cdot \xi^{\prime}\right) \xi_{k}, \quad k=1,2 \\
V_{3} & =i\left(i \hat{b}_{3}+\frac{1}{s} \hat{b}^{\prime} \cdot \xi^{\prime}\right)\left(\tau_{2}-\tau_{1}\right)=-\frac{i c_{0}}{\tau_{1}+\tau_{2}}\left(i s \hat{b}_{3}+\hat{b}^{\prime} \cdot \xi^{\prime}\right) \\
W_{k} & =\Phi_{k}+\Psi \xi_{k}=\frac{1}{s}\left(\frac{1}{\tau_{2}}-\frac{1}{\tau_{1}}\right) \hat{b}^{\prime} \cdot \xi^{\prime} \xi_{k}-\frac{1}{\mu \tau_{1}} \hat{b}_{k}+\frac{i}{\tau_{2}} \hat{b}_{3} \xi_{k} \\
& =\frac{c_{0}}{\tau_{1} \tau_{2}\left(\tau_{1}+\tau_{2}\right)} \hat{b}^{\prime} \cdot \xi^{\prime} \xi_{k}-\frac{1}{\mu \tau_{1}} \hat{b}_{k}+\frac{i}{\tau_{2}} \hat{b}_{3} \xi_{k}, \quad k=1,2 \\
W_{3} & =\hat{b}_{3}
\end{aligned}
$$

where $c_{0}=\frac{\nu}{\mu(\nu+\mu)}$. Hence

$$
\hat{u}_{m}=\sum_{r=1}^{3}\left(g_{m r} e_{0} \hat{b}_{r}+h_{m r} e_{1} \hat{b}_{r}\right), \quad m=1,2,3
$$


where

$$
\begin{aligned}
& g_{11}=\frac{-c_{0} \xi_{1}^{2}}{\tau_{2}\left(\tau_{1}+\tau_{2}\right)}, \quad g_{22}=\frac{-c_{0} \xi_{2}^{2}}{\tau_{2}\left(\tau_{1}+\tau_{2}\right)}, \quad g_{12}=g_{21}=\frac{-c_{0} \xi_{1} \xi_{2}}{\tau_{2}\left(\tau_{1}+\tau_{2}\right)}, \\
& g_{13}=\frac{-c_{0} \xi_{1} s i}{\tau_{2}\left(\tau_{1}+\tau_{2}\right)}, \quad g_{23}=\frac{-c_{0} \xi_{2} s i}{\tau_{2}\left(\tau_{1}+\tau_{2}\right)}, \\
& g_{31}=\frac{-i c_{0} \xi_{1}}{\tau_{1}+\tau_{2}}, \quad g_{32}=\frac{-i c_{0} \xi_{2}}{\tau_{1}+\tau_{2}}, \quad g_{33}=\frac{-c_{0} s}{\tau_{1}+\tau_{2}}, \\
& h_{11}=-\frac{1}{\mu \tau_{1}}+\frac{c_{0} \xi_{1}^{2}}{\tau_{1} \tau_{2}\left(\tau_{1}+\tau_{2}\right)}, \quad h_{12}=h_{21}=\frac{c_{0} \xi_{1} \xi_{2}}{\tau_{1} \tau_{2}\left(\tau_{1}+\tau_{2}\right)}, \\
& h_{22}=-\frac{1}{\mu \tau_{1}}+\frac{c_{0} \xi_{2}^{2}}{\tau_{1} \tau_{2}\left(\tau_{1}+\tau_{2}\right)}, \quad h_{13}=\frac{i \xi_{1}}{\tau_{2}}, \\
& h_{23}=\frac{i \xi_{2}}{\tau_{2}}, \quad h_{31}=h_{32}=0, \quad h_{33}=1 .
\end{aligned}
$$

LEMma 3.1. Let $\tau=\sqrt{\gamma+\xi^{\prime 2}+i \xi_{0}}$. Then the following estimates hold:

$$
\begin{array}{ll}
\left|\partial_{\xi^{\prime}}^{r^{\prime}} \partial_{\xi_{0}}^{r} g_{j k}\right| \leq \frac{c}{|\tau|^{r^{\prime}+2 r}}, \quad r^{\prime}, r \in \mathbb{N} \cup\{0\}, \quad j=1,2,3, \quad k=1,2 ; \\
\left|\partial_{\xi^{\prime}}^{r^{\prime}} \partial_{\xi_{0}}^{r} g_{j 3}\right| \leq \frac{c}{|\tau|^{r^{\prime}+2 r-1}}, \quad r^{\prime}, r \in \mathbb{N} \cup\{0\}, \quad j=1,2,3 ; \\
\left|\partial_{\xi^{\prime}}^{r^{\prime}} \partial_{\xi_{0}}^{r} h_{j k}\right| \leq \frac{c}{|\tau|^{r^{\prime}+2 r+1}}, \quad r^{\prime}, r \in \mathbb{N} \cup\{0\}, \quad j, k=1,2 ; \\
\left|\partial_{x_{i^{\prime}}}^{r^{\prime}} \partial_{\xi_{0}}^{r} h_{j 3}\right| \leq \frac{c}{|\tau|^{r^{\prime}+2 r}}, \quad r^{\prime}, r \in \mathbb{N} \cup\{0\}, \quad j=1,2 .
\end{array}
$$

Proof. First, notice that

$$
\begin{aligned}
& c_{1}|\tau| \leq\left|\tau_{1}\right| \leq c_{2}|\tau|, \\
& c_{3}|\tau| \leq\left|\tau_{2}\right| \leq c_{4}|\tau|,
\end{aligned}
$$

where $c_{j}>0(j=1, \ldots, 4)$ are constants, and $\tau_{j}, j=1,2$, can be written as

$$
\tau_{j}=\left|\tau_{j}\right|\left(\cos \varphi_{j}+i \sin \varphi_{j}\right),
$$

where $\varphi_{j}=\arg \tau_{j} \in\left(-\frac{\pi}{4}, \frac{\pi}{4}\right), j=1,2$. This implies that

$$
\left|\tau_{1}+\tau_{2}\right|=\sqrt{\left|\tau_{1}\right|^{2}+\left|\tau_{2}\right|^{2}+2\left|\tau_{1}\right|\left|\tau_{2}\right| \cos \left(\varphi_{1}-\varphi_{2}\right)}
$$

Hence

$$
c_{5}|\tau| \leq\left|\tau_{1}+\tau_{2}\right| \leq c_{6}|\tau|
$$

where $c_{j}>0(j=5,6)$ are constants and we used that $\cos \left(\varphi_{1}-\varphi_{2}\right)>0$ and (3.6)-(3.7).

Moreover

$$
\left|\partial_{\xi^{\prime}}^{r^{\prime}} \partial_{\xi_{0}}^{r} \tau_{j}\right| \leq \frac{c}{\left|\tau_{j}\right|^{r^{\prime}+2 r-1}}, \quad j=1,2
$$

In view of (3.6)-(3.9) we can replace calculation of derivatives of the functions $g_{j k}$ and 
$h_{j k}(j, k=1,2,3)$ by calculating derivatives of the following functions:

$$
\begin{aligned}
& g_{11}^{1}=\frac{\xi_{1}^{2}}{\tau^{2}}, \quad g_{22}^{1}=\frac{\xi_{2}^{2}}{\tau^{2}}, \quad g_{12}^{1}=g_{21}^{1}=\frac{\xi_{1} \xi_{2}}{\tau^{2}}, \quad g_{13}^{1}=\frac{\xi_{1} s}{\tau^{2}}, \quad g_{23}^{1}=\frac{\xi_{2} s}{\tau^{2}}, \\
& g_{31}^{1}=\frac{\xi_{1}}{\tau}, \quad g_{32}^{1}=\frac{\xi_{2}}{\tau}, \quad g_{33}^{1}=\frac{s}{\tau}, \quad h_{11}^{1}=\frac{1}{\tau}+\frac{\xi_{1}^{2}}{\tau^{3}}, \\
& h_{12}^{1}=h_{21}^{1}=\frac{\xi_{1} \xi_{2}}{\tau^{3}}, \quad h_{22}^{1}=\frac{1}{\tau}+\frac{\xi_{2}^{2}}{\tau^{3}}, \quad h_{13}^{1}=\frac{\xi_{1}}{\tau}, \quad h_{23}^{1}=\frac{\xi_{2}}{\tau} .
\end{aligned}
$$

To calculate the derivatives of the above functions we use the formula:

$$
\partial_{\xi^{\prime}}^{r^{\prime}} \partial_{\xi_{0}}^{r}\left(\tau^{-m}\right)=\sum_{l=0}^{r+r^{\prime}-1} a_{l+1} \tau^{-m-(l+1)} \cdot \sum_{\substack{n_{1}+\cdots+n_{q}=l+1 \\ m_{1} n_{1}+\cdots+m_{q} n_{q}=r \\ m_{1}^{\prime} n_{1}+\cdots+m_{q}^{\prime} n_{q}=r^{\prime}}}\left(\partial_{\xi_{0}}^{m_{1}} \partial_{\xi^{\prime}}^{m_{1}^{\prime}} \tau\right)^{n_{1}} \cdots\left(\partial_{\xi_{0}}^{m_{q}} \partial_{\xi^{\prime}}^{m_{q}^{\prime}} \tau\right)^{n_{q}},
$$

where $m \in \mathbb{R} \backslash\{0\}, a_{l+1}\left(l=0, \ldots, r+r^{\prime}-1\right)$ are real constnts depending on $k+k^{\prime}$, $\partial_{\xi^{\prime}}^{k^{\prime}}=\partial_{\xi_{1}}^{k_{1}^{\prime}} \partial_{\xi_{2}}^{k_{2}^{\prime}} ; m_{j}, m_{j}^{\prime}$ and $n_{j}(j=1, \ldots, q)$ are nonnegative integer numbers. Using (3.10) and the Leibniz formula we get

$$
\begin{aligned}
\left|\partial_{\xi^{\prime}}^{r^{\prime}} \partial_{\xi_{0}}^{r} g_{11}^{1}\right|= & \left|\sum_{l=0}^{r^{\prime}}\left(\begin{array}{c}
r^{\prime} \\
l
\end{array}\right) \partial_{\xi_{1}}^{l}\left(\xi_{1}^{2}\right) \partial_{\xi^{\prime}}^{r^{\prime}-l} \partial_{\xi_{0}}^{r}\left(\tau^{-2}\right)\right| \\
\leq & c \sum_{l=0}^{r^{\prime}}\left(|\tau|^{-2-(l+1)}|\tau|^{l+1-\left(2 r+r^{\prime}\right)}\left|\xi_{1}\right|^{2}+|\tau|^{-2-(l+1)}|\tau|^{l+1-\left(2 r+r^{\prime}-1\right)}\left|\xi_{1}\right|\right. \\
& \left.+|\tau|^{-2-(l+1)}|\tau|^{l+1-\left(2 r+r^{\prime}-2\right)}\right) \leq \frac{c}{|\tau|^{2 r+r^{\prime}}},
\end{aligned}
$$

where we used that

$$
\left|\partial_{\xi_{0}}^{m_{j}} \partial_{\xi^{\prime}}^{m_{j}^{\prime}} \tau\right|^{n_{j}} \leq c|\tau|^{n_{j}-n_{j}\left(m_{j}^{\prime}+2 m_{j}\right)}, \quad j=1, \ldots, q .
$$

Hence

$$
\left|\partial_{\xi^{\prime}}^{r^{\prime}} \partial_{\xi_{0}}^{r} g_{11}\right| \leq \frac{c}{|\tau|^{2 r+r^{\prime}}}
$$

Using (3.6)-(3.9), the formula (3.10) and the Leibniz formula we estimate the other functions $g_{j k}$ and $h_{j k}$ in the same way.

This concludes the proof.

We also need some estimates of derivatives $\partial_{x_{3}}^{j} \partial_{\xi_{0}}^{k} \partial_{\xi^{\prime}}^{k^{\prime}} e_{i}, i=0,1$ which will be proved in Lemmas 3.2 and 3.3 below.

Lemma 3.2. Let $\tau^{2}=s+\xi^{\prime 2}, s=\gamma+i \xi_{0}, \xi^{\prime}=\left(\xi_{1}, \xi_{2}\right) \in \mathbb{R}^{2}, \xi_{0} \in \mathbb{R}, \gamma>0$. Then

$$
\begin{gathered}
\int_{0}^{\infty}\left|\partial_{x_{3}}^{j} \partial_{\xi_{0}}^{k} \partial_{\xi^{\prime}}^{k^{\prime}} e_{1}\right|^{p} d x_{3} \leq c|\tau|^{p j-p\left(2 k+k^{\prime}\right)-1}, \\
\int_{0}^{\infty}\left|\partial_{x_{3}}^{j} \partial_{\xi_{0}}^{k} \partial_{\xi^{\prime}}^{k^{\prime}} e_{0}\right|^{p} d x_{3} \leq c|\tau|^{p j-p\left(2 k+k^{\prime}\right)-p-1},
\end{gathered}
$$

where $p \in(1, \infty), j, k, k^{\prime} \in \mathbb{N} \cup\{0\}, \partial_{\xi^{\prime}}^{k^{\prime}}=\partial_{\xi_{1}}^{k_{1}} \partial_{\xi_{2}}^{k_{2}}, k^{\prime}=k_{1}+k_{2} ; c>0$ does not depend on $\tau$. 
Proof. By Lemma 2.2 of [27]

$$
\int_{0}^{\infty}\left|\partial_{x_{3}}^{j} \partial_{\xi_{0}}^{k} \partial_{\xi^{\prime}}^{k^{\prime}} e^{-\tau_{1} x_{3}}\right|^{p} d x_{3} \leq c\left|\tau_{1}\right|^{p j-p\left(2 k+k^{\prime}\right)-1}
$$

and in view of (3.6), estimate (3.11) follows. In order to prove (3.12) notice that

$$
\partial_{x_{3}}^{j} e_{0}=\left(-\tau_{2}\right)^{j} e_{0}+(-1)^{j}\left(\tau_{1}^{j-1}+\tau_{1}^{j-2} \tau_{2}+\cdots+\tau_{2}^{j-1}\right) e_{1} \equiv A_{1}+A_{2},
$$

where $A_{1}=\left(-\tau_{2}\right)^{j} e_{0}$. First, using relations (3.7), (3.8), (3.10) it suffices to estimate $\partial_{\xi_{0}}^{k} \partial_{\xi^{\prime}}^{k^{\prime}}\left((-1)^{j} \tau^{j-1} e^{-\tau x_{3}}\right)$ instead of $\partial_{\xi_{0}}^{k} \partial_{\xi^{\prime}}^{k^{\prime}} A_{2}$. Since for $j \in \mathbb{N}$,

$$
\partial_{\xi_{0}}^{k} \partial_{\xi^{\prime}}^{k^{\prime}}\left((-1)^{j} \tau^{j-1} e^{-\tau x_{3}}\right)=-\partial_{x_{3}}^{j-1} \partial_{\xi_{0}}^{k} \partial_{\xi^{\prime}}^{k^{\prime}} e^{-\tau x_{3}},
$$

in view of (3.11) we have

$$
\int_{0}^{\infty}\left|\partial_{\xi_{0}}^{k} \partial_{\xi^{\prime}}^{k^{\prime}} A_{2}\right|^{p} d x_{x} \leq c|\tau|^{p j-p\left(2 k+k^{\prime}\right)-p-1} .
$$

If $j=0$ we apply the Leibniz formula together with (3.10) for $m=1$ and the formula

$$
\partial_{\xi_{0}}^{k} \partial_{\xi^{\prime}}^{k^{\prime}} e^{-\tau x_{3}}=e^{-\tau x_{3}} \sum_{l=0}^{k+k^{\prime}-1} a_{l+1} x_{3}^{l+1}
$$

$$
\sum_{\substack{n_{1}+\cdots+n_{s}=l+1 \\ m_{1} n_{1}+\cdots+m_{s} n_{s}=k \\ m_{1} n_{1}+\cdots+m_{s}^{\prime} n_{s}=k^{\prime}}}\left(\partial_{\xi_{0}}^{m_{1}} \partial_{\xi^{\prime}}^{m_{1}^{\prime}} \tau\right)^{q_{1}} \cdots\left(\partial_{\xi_{0}}^{m_{s}} \partial_{\xi^{\prime}}^{m_{s}^{\prime}} \tau\right)^{q_{s}}
$$

where $a_{i}\left(i=1, \ldots, k+k^{\prime}\right)$ are nonnegative constants. Then it follows that $(3.14)$ holds also for $j=0$.

In order to estimate $\partial_{\xi_{0}}^{k} \partial_{\xi^{\prime}}^{k^{\prime}} A_{1}$ we represent $e_{0}$ in the form

$$
e_{0}\left(x_{3}\right)=-\int_{0}^{x_{3}} e^{-\tau_{1}\left(x_{3}-y\right)} e^{-\tau_{2} y} d y
$$

Then

$$
\begin{aligned}
& \partial_{\xi^{\prime}} e_{0}\left(x_{3}\right)=-\int_{0}^{x_{3}}\left(\partial_{\xi^{\prime}} e^{-\tau_{1}\left(x_{3}-y\right)} e^{-\tau_{2} y}+e^{-\tau_{1}\left(x_{3}-y\right)} \partial_{\xi^{\prime}} e^{-\tau_{2} y}\right) d y \\
& =\partial_{\xi^{\prime}} \tau_{1} \int_{0}^{x_{3}}\left(x_{3}-y\right) e^{-\tau_{1}\left(x_{3}-y\right)} e^{-\tau_{2} y} d y+\partial_{\xi^{\prime}} \tau_{2} \int_{0}^{x_{3}} y e^{-\tau_{2} y} e^{-\tau_{1}\left(x_{3}-y\right)} d y
\end{aligned}
$$

Therefore, using (3.6)-(3.7), (3.9) we get

$$
\begin{aligned}
& \left|\partial_{\xi^{\prime}} e_{0}\left(x_{3}\right)\right| \leq\left|\partial_{\xi^{\prime}} \tau_{1}\right| \sup _{0<y<\infty}\left(\left|x_{3}-y\right| e^{-\frac{R e \tau_{1}\left|x_{3}-y\right|}{2}}\right) \int_{0}^{x_{3}} e^{-\frac{R e \tau_{1}}{2}\left(x_{3}-y\right)} e^{-R e \tau_{2} y} d y \\
& \quad+\left|\partial_{\xi^{\prime}} \tau_{2}\right| \sup _{0<y<\infty}\left(y e^{-\frac{R e \tau_{2}}{2} y}\right) \int_{0}^{x_{3}} e^{-\frac{R e \tau_{2}}{2} y} e^{-R e \tau_{1}\left(x_{3}-y\right)} d y \\
& \leq\left|\partial_{\xi^{\prime}} \tau_{1}\right| \sup _{0<z<\infty}\left(z e^{-\frac{R e \tau_{1}}{2} z}\right) \int_{0}^{x_{3}} e^{-\frac{R e \tau_{1}}{2}\left(x_{3}-y\right)} e^{-R e \tau_{2} y} d y \\
& \quad+\left|\partial_{\xi^{\prime}} \tau_{2}\right| \sup _{0<y<\infty}\left(y e^{-\frac{R e \tau_{2}}{2} y}\right) \int_{0}^{x_{3}} e^{-R e \tau_{1}\left(x_{3}-y\right)} e^{-\frac{R e \tau_{2}}{2} y} d y \\
& \leq \frac{c}{|\tau|}\left(\int_{0}^{x_{3}} e^{-\frac{R e \tau_{1}}{2}\left|x_{3}-y\right|} e^{-R e \tau_{2}|y|} d y+\int_{0}^{x_{3}} e^{-\frac{R e \tau_{2}}{2}|y|} e^{-R e \tau_{1}\left|x_{3}-y\right|} d y\right)
\end{aligned}
$$


Hence by the Young inequality we obtain

$$
\begin{aligned}
& \int_{0}^{\infty}\left|\partial_{\xi^{\prime}} e_{0}\left(x_{3}\right)\right|^{p} d x_{3} \leq \frac{c}{|\tau|^{p}}\left[\left(\int_{0}^{\infty} e^{-R e \tau_{2} y} d y\right)^{p} \int_{0}^{\infty} e^{-p \frac{R e \tau_{1}}{2} x_{3}} d x_{3}\right. \\
& \left.+\left(\int_{0}^{\infty} e^{-\frac{R e \tau_{2}}{2} y} d y\right)^{p} \int_{0}^{\infty} e^{-p R e \tau_{1} x_{3}} d x_{3}\right] \leq \frac{c}{|\tau|^{2 p+1}}
\end{aligned}
$$

where we also used that $\left|\tau_{i}\right|<\frac{\sqrt{2}}{2} \operatorname{Re} \tau_{i}, i=1,2$. In the same way we get

$$
\int_{0}^{\infty}\left|\partial_{\xi_{0}} e_{0}\left(x_{3}\right)\right|^{p} d x_{3} \leq \frac{c}{|\tau|^{3 p+1}} .
$$

Now, we estimate $\int_{0}^{\infty}\left|\partial_{\xi^{\prime}}^{k^{\prime}} \partial_{\xi_{0}}^{k} e_{0}\left(x_{3}\right)\right|^{p} d x$, where $k+k^{\prime} \geq 2, k, k^{\prime} \in \mathbb{N} \cup\{0\}$.

To do this we use the formulas

$$
\begin{aligned}
& \partial_{\xi_{0}}^{r} \partial_{\xi^{\prime}}^{r^{\prime}} e^{-\tau_{1}\left(x_{3}-y\right)}=e^{-\tau_{1}\left(x_{3}-y\right)} \sum_{l=0}^{r^{\prime}+r-1} b_{l+1}\left(x_{3}-y\right)^{l+1} \\
& \sum_{\substack{n_{1}+\cdots+n_{s}=l+1 \\
m_{1} n_{1}+\cdots+m_{s} n_{s}=r \\
m_{1}^{\prime} n_{1}+\cdots+m_{s}^{\prime} n_{s}=r^{\prime}}}\left(\partial_{\xi_{0}}^{m_{1}} \partial_{\xi^{\prime}}^{m_{1}^{\prime}} \tau_{1}\right)^{n_{1}}\left(\partial_{\xi_{0}}^{m_{s}} \partial_{\xi^{\prime}}^{m_{s}^{\prime}} \tau_{1}\right)^{n_{s}}
\end{aligned}
$$

and

$$
\partial_{\xi_{0}}^{k-r} \partial_{\xi^{\prime}}^{k^{\prime}-r^{\prime}} e^{-\tau_{2} y}=e^{-\tau_{2} y} \sum_{\substack{m=0 \\ m^{\prime}}}^{k+k^{\prime}-r-r^{\prime}-1} \sum_{\substack{m+1 \\ m_{1}+\cdots+n_{q}=m+1 \\ m_{1}^{\prime} n_{1}+\cdots+m_{q} n_{q}=r-k \\ m_{1}+\cdots+m_{q}^{\prime} n_{q}=r^{\prime}-k^{\prime}}}\left(\partial_{\xi_{0}}^{m_{1}} \partial_{\xi^{\prime}}^{m_{1}^{\prime}} \tau_{2}\right)^{n_{1}} \ldots\left(\partial_{\xi_{0}}^{m_{q}} \partial_{\xi^{\prime}}^{m^{\prime}} \tau_{2}\right)^{n_{q}},
$$

where $r<k, r^{\prime}<k^{\prime}, r-k \in \mathbb{N}, r^{\prime}-k^{\prime} \in \mathbb{N}, b_{i}$ and $c_{j}\left(i=1, \ldots, r^{(1)}+r ; j=\right.$ $\left.1, \ldots, k+k^{\prime}-r-r^{\prime}\right)$ are nonnegative constants. Then applying also the Leibniz formula and estimates (3.6), (3.7), (3.9) we obtain

$$
\begin{aligned}
\left|\partial_{\xi_{0}}^{k} \partial_{\xi^{\prime}}^{k^{\prime}} e_{0}\left(x_{3}\right)\right| \leq & c \int_{0}^{x_{3}}\left(\left|\partial_{\xi_{0}}^{k} \partial_{\xi^{\prime}}^{k^{\prime}} e^{-\tau_{1}\left(x_{3}-y\right)}\right|\left|e^{-\tau_{2} y}\right|+\left|e^{-\tau_{1}\left(x_{3}-y\right)}\right|\left|\partial_{\xi_{0}}^{k} \partial_{\xi^{\prime}}^{k^{\prime}} e^{-\tau_{2} y}\right|\right) d y \\
& +\sum_{r+r^{\prime}=1}^{k+k^{\prime}-1} \int_{0}^{x_{3}}\left|\partial_{\xi_{0}}^{r} \partial_{\xi^{\prime}}^{r^{\prime}} e^{-\tau_{1}\left(x_{3}-y\right)}\right|\left|\partial_{\xi_{0}}^{r-k} \partial_{\xi^{\prime}}^{r^{\prime}-k^{\prime}} e^{-\tau_{2} y}\right| d y \\
\leq & c|\tau|^{-k^{\prime}-2 k}\left[\int_{0}^{x_{3}} e^{-\operatorname{Re} \tau_{1}\left(x_{3}-y\right)} e^{-\operatorname{Re} \tau_{2} y} \sum_{l=0}^{k+k^{\prime}-1}\left(x_{3}-y\right)^{l+1}|\tau|^{l+1} d y\right. \\
& \left.+\int_{0}^{x_{3}} e^{-\operatorname{Re} \tau_{1}\left(x_{3}-y\right)} e^{-\operatorname{Re} \tau_{2} y} \sum_{l=0}^{k+k^{\prime}-1} y^{l+1}|\tau|^{l+1} d y\right] \\
& +\sum_{r+r^{\prime}=1}^{k+k^{\prime}-1} \int_{0}^{x_{3}}\left[e^{-R e \tau_{1}\left(x_{3}-y\right)} \sum_{l=0}^{r+r^{\prime}-1}\left(x_{3}-y\right)^{l+1}|\tau|^{-r^{\prime}-2 r+(l+1)}\right. \\
& \left.\cdot e^{-\operatorname{Re}_{2} y} \sum_{m=0}^{k+k^{\prime}-r-r^{\prime}-1} y^{m+1}|\tau|^{-\left(k^{\prime}-r^{\prime}\right)-2(k-r)+m+1}\right] d y .
\end{aligned}
$$


Continuing, we have

$$
\begin{aligned}
& \left|\partial_{\xi_{0}}^{k} \partial_{\xi^{\prime}}^{k^{\prime}} e_{0}\left(x_{3}\right)\right| \leq c|\tau|^{-k^{\prime}-2 k}\left\{\sum _ { l = 0 } ^ { k + k ^ { \prime } - 1 } | \tau | ^ { l + 1 } \left[\int_{0}^{x_{3}} e^{-\operatorname{Re} \tau_{1}\left(x_{3}-y\right)} \cdot e^{-\operatorname{Re} \tau_{2} y}\left(x_{3}-y\right)^{l+1} d y\right.\right. \\
& \left.\quad+\int_{0}^{x_{3}} e^{-\operatorname{Re} \tau_{1}\left(x_{3}-y\right)} e^{-\operatorname{Re} \tau_{2} y} y^{l+1} d y\right] \\
& \left.\quad+\sum_{m=1}^{k+k^{\prime}} \sum_{l=1}^{k+k^{\prime}-m} \int_{0}^{x_{3}} e^{-\operatorname{Re} \tau_{1}\left(x_{3}-y\right)} e^{-\operatorname{Re} \tau_{2} y}\left(x_{3}-y\right)^{m} y^{l}|\tau|^{m+l} d y\right\}
\end{aligned}
$$

Hence

$$
\begin{gathered}
\left|\partial_{\xi_{0}}^{k} \partial_{\xi^{\prime}}^{k^{\prime}} e_{0}\left(x_{3}\right)\right| \leq c|\tau|^{-k^{\prime}-2 k}\left\{\sum_{l=0}^{k+k^{\prime}-1}|\tau|^{l+1}\right. \\
\cdot\left[\sup _{0<y<\infty}\left(\left|x_{3}-y\right|^{l+1} e^{-\frac{R e \tau_{1}}{2}\left(x_{3}-y\right)}\right) \int_{0}^{x_{3}} e^{-\frac{R e \tau_{1}}{2}\left(x_{3}-y\right)} e^{-R e \tau_{2} y} d y\right. \\
\left.+\sup _{0<y<\infty}\left(y^{l+1} e^{-\frac{R e \tau_{1}}{2} y}\right) \int_{0}^{x_{3}} e^{-R e \tau_{1}\left(x_{3}-y\right)} e^{-\frac{R e \tau_{2}}{2} y} d y\right] \\
+\sum_{m=1}^{k+k^{\prime}} \sum_{l=1}^{k+k^{\prime}-m}|\tau|^{m+l} \sup _{0<y<\infty}\left(\left|x_{3}-y\right|^{m} e^{-\frac{R e \tau_{1}}{2}\left(x_{3}-y\right)}\right) \\
\left.\cdot \sup _{0<y<\infty}\left(y^{l} e^{-\frac{R e \tau_{2}}{2} y}\right) \int_{0}^{x_{3}} e^{-\frac{R e \tau_{1}}{2}\left(x_{3}-y\right)} e^{-\frac{R e \tau_{2}}{2} y} d y\right\} .
\end{gathered}
$$

Since

$$
\sup _{0<t<\infty}\left(t^{r} e^{-\frac{R e \tau_{i}}{2} t}\right) \leq c|\tau|^{-r}, \quad r \in \mathbb{N}, i=1,2,
$$

we get

$$
\begin{aligned}
& \left|\partial_{\xi_{0}}^{k} \partial_{\xi^{\prime}}^{k^{\prime}} e_{0}\left(x_{3}\right)\right| \leq c|\tau|^{-k^{\prime}-2 k}\left[\int_{0}^{x_{3}} e^{-\frac{R e \tau_{1}}{2}\left(x_{3}-y\right)} e^{-R e \tau_{2} y} d y\right. \\
& \left.\quad+\int_{0}^{x_{3}} e^{-\frac{R e \tau_{2}}{2} y} e^{-R e \tau_{1}\left(x_{3}-y\right)} d y+\int_{0}^{x_{3}} e^{-\frac{R e \tau_{1}}{2}\left(x_{3}-y\right)} e^{-\frac{R e \tau_{2}}{2} y} d y\right] .
\end{aligned}
$$

Therefore, using as before the Young inequality yields

$$
\int_{0}^{\infty}\left|\partial_{\xi_{0}}^{k} \partial_{\xi^{\prime}}^{k^{\prime}} e_{0}\left(x_{3}\right)\right|^{p} d x_{3} \leq c|\tau|^{-\left(2 k+k^{\prime}\right) p-p-1}, \quad k, k^{\prime} \in \mathbb{N} \cup\{0\} .
$$

Now, applying once more the Leibniz formula and (3.16) we obtain

$$
\begin{aligned}
& \int_{0}^{\infty}\left|\partial_{\xi_{0}}^{k} \partial_{\xi^{\prime}}^{k^{\prime}} A_{1}\right|^{p} d x_{3} \leq c \sum_{r=0}^{k} \sum_{r^{\prime}=0}^{k^{\prime}} \int_{0}^{\infty}|\tau|^{p\left[j-(k-r)-2\left(k^{\prime}-r^{\prime}\right)\right]}\left|\partial_{\xi_{0}}^{r} \partial_{\xi^{\prime}}^{r^{\prime}} e_{0}\left(x_{3}\right)\right|^{p} d x_{3} \\
& \leq c|\tau|^{p j-p\left(2 k+k^{\prime}\right)-p-1} .
\end{aligned}
$$

In view of (3.14) and (3.17) estimate (3.12) follows.

We will also use the following lemma: 
Lemma 3.3. Let the assumptions of Lemma 3.2 be satisfied. Then

$$
\begin{aligned}
& \int_{0}^{\infty} \int_{0}^{\infty} \frac{\left|\partial_{x_{3}}^{j} \partial_{\xi_{0}}^{k} \partial_{\xi^{\prime}}^{k^{\prime}} e_{1}\left(x_{3}+z\right)-\partial_{x_{3}}^{j} \partial_{\xi_{0}}^{k} \partial_{\xi^{\prime}}^{k^{\prime}} e_{1}\left(x_{3}\right)\right|^{p}}{z^{1+p \varkappa}} d x_{3} d z \\
& \leq c|\tau|^{p(j+\varkappa)-p\left(2 k+k^{\prime}\right)-1}, \\
& \int_{0}^{\infty} \int_{0}^{\infty} \frac{\left|\partial_{x_{3}}^{j} \partial_{\xi_{0}}^{k} \partial_{\xi^{\prime}}^{k^{\prime}} e_{0}\left(x_{3}+z\right)-\partial_{x_{3}}^{j} \partial_{\xi_{0}}^{k} \partial_{\xi^{\prime}}^{k^{\prime}} e_{0}\left(x_{0}\right)\right|^{p}}{z^{1+p \varkappa}} d x_{3} d z \\
& \leq c|\tau|^{p(j+\varkappa)-p\left(2 k+k^{\prime}\right)-p-1},
\end{aligned}
$$

where $\varkappa \in(0,1)$.

Proof. By Lemma 2.2 of [27] and (3.6),

$$
\int_{0}^{\infty} \int_{0}^{\infty} \frac{\left|\partial_{x_{3}}^{j} \partial_{\xi_{0}}^{k} \partial_{\xi^{\prime}}^{k^{\prime}} e^{-\tau_{1}\left(x_{3}+z\right)}-\partial_{x_{3}}^{j} \partial_{\xi_{0}}^{k} \partial_{\xi^{\prime}}^{k^{\prime}} e^{-\tau_{1} x_{3}}\right|^{p}}{z^{1+p \varkappa}} d x_{3} d z \leq c|\tau|^{p(j+\varkappa)-p\left(2 k+k^{\prime}\right)-1}
$$

so estimate (3.18) follows. It remains to prove (3.19). First, let $k=k^{\prime}=j=0$. Then

$$
e_{0}\left(x_{3}+z\right)-e_{0}\left(x_{3}\right)=e^{-\tau_{1} x_{3}} e_{0}(z)+e_{0}\left(x_{3}\right)\left(e^{-\tau_{2} z}-1\right)
$$

and we have

$$
\begin{aligned}
& \int_{0}^{\infty} \int_{0}^{\infty} \frac{\left|e_{0}\left(x_{3}+z\right)-e_{0}\left(x_{3}\right)\right|^{p}}{z^{1+p \varkappa}} d x_{3} d z \leq \int_{0}^{\infty} e^{-p R e \tau_{1} x_{3}} d x_{3} \cdot \int_{0}^{\infty}\left|e_{0}(z)\right|^{p} \frac{d z}{z^{1+p \varkappa}} \\
& \quad+\int_{0}^{\infty}\left|e_{0}\left(x_{3}\right)\right|^{p} d x_{3} \int_{0}^{\infty} \frac{\left|e^{-\tau_{2} z}-1\right|^{p}}{z^{1+p \varkappa}} d z \\
& \leq \frac{c}{|\tau|} \int_{0}^{\infty} \frac{\left|e_{0}(z)\right|^{p}}{z^{1+p \varkappa}} d z+\frac{c}{|\tau|^{p+1}} \int_{0}^{\infty} \frac{\left|e^{-\tau_{2} z}-1\right|^{p}}{z^{1+p \varkappa}} d z .
\end{aligned}
$$

Since

$$
\int_{0}^{\infty} \frac{\left|e_{0}(z)\right|^{p}}{z^{1+p \varkappa}} d z=\int_{0}^{\infty} \frac{e^{-p \operatorname{Re} \tau_{2} z}}{z^{1+p \varkappa}}\left|\frac{e^{-\left(\tau_{1}-\tau_{2}\right) z}-1}{\tau_{1}-\tau_{2}}\right|^{p} d z \leq \int_{0}^{\infty} z^{p-1-p \varkappa} e^{-p \operatorname{Re} \tau_{2} z} d z \leq c|\tau|^{p \varkappa-p}
$$

and

$$
\int_{0}^{\infty} \frac{\left|e^{-\tau_{2} z}-1\right|^{p}}{z^{1+p \varkappa}} d z \leq c|\tau|^{p \varkappa}
$$

we obtain

$$
\int_{0}^{\infty} \frac{\left|e_{0}\left(x_{3}+z\right)-e_{0}\left(x_{3}\right)\right|^{p}}{z^{1+p \varkappa}} d x_{3} d z \leq c|\tau|^{p \varkappa-p-1} .
$$

Now, let $k, k^{\prime}, j \in \mathbb{N}$. By (3.13)

$$
\begin{aligned}
& \partial_{x_{3}}^{j} e_{0}\left(x_{3}+z\right)-\partial_{x_{3}}^{j} e_{0}\left(x_{3}\right)=\left(-\tau_{2}\right)^{j}\left[e_{0}\left(x_{3}+z\right)-e_{0}\left(x_{3}\right)\right] \\
& \quad+(-1)^{j}\left(\tau_{1}^{j-1}+\tau_{1}^{j-1} \tau_{2}+\cdots+\tau_{2}^{j-1}\right)\left[e_{1}\left(x_{3}+z\right)-e_{1}\left(x_{3}\right)\right] \\
& =A_{1}\left(x_{3}+z\right)-A_{1}\left(x_{3}\right)+A_{2}\left(x_{3}+z\right)-A_{2}\left(x_{3}\right) .
\end{aligned}
$$

Instead of $\partial_{\xi_{0}}^{k} \partial_{\xi^{\prime}}^{k^{\prime}}\left(A_{2}\left(x_{3}+z\right)-A_{2}\left(x_{3}\right)\right)$ we estimate $\partial_{\xi_{0}}^{k} \partial_{\xi^{\prime}}^{k^{\prime}}\left(A_{2}^{1}\left(x_{3}+z\right)-A_{2}^{1}\left(x_{3}\right)\right)$, where $A_{2}^{1}=(-1)^{j} \tau^{j-1} e^{-\tau x_{3}}$. Then, in view of (3.18) with $j$ replaced by $j-1$ and $\tau_{1}$ replaced 
by $\tau$ we get

$$
\int_{0}^{\infty} \int_{0}^{\infty} \frac{\left|\partial_{\xi_{0}}^{k} \partial_{\xi^{\prime}}^{k^{\prime}} A_{2}\left(x_{3}+z\right)-\partial_{\xi_{0}}^{k} \partial_{\xi^{\prime}}^{k^{\prime}} A_{2}\left(x_{3}\right)\right|^{p}}{z^{1+p \varkappa}} d x_{3} d z
$$

$$
\leq c|\tau|^{p(j+\varkappa)-p\left(2 k+k^{\prime}\right)-p-1} .
$$

To estimate $\partial_{\xi_{0}}^{k} \partial_{\xi^{\prime}}^{k^{\prime}}\left(A_{1}\left(x_{3}+z\right)-A_{1}\left(x_{3}\right)\right)$ we write

$$
\begin{aligned}
& e_{0}\left(x_{3}+z\right)-e_{0}\left(x_{3}\right)=-\int_{0}^{x_{3}+z} e^{-\tau_{1}\left(x_{3}+z-y\right)} e^{-\tau_{2} y} d y+\int_{0}^{x_{3}} e^{-\tau_{1}\left(x_{3}-y\right)} e^{-\tau_{2} y} d y \\
& =-\int_{x_{3}}^{x_{3}+z} e^{-\tau_{1}\left(x_{3}+z-y\right)} e^{-\tau_{2} y} d y-\int_{0}^{x_{3}}\left(e^{-\tau_{1}\left(x_{3}+z-y\right)}-e^{-\tau_{1}\left(x_{3}-y\right)}\right) e^{-\tau_{2} y} d y .
\end{aligned}
$$

Hence, by using the Leibniz formula we get

$$
\begin{aligned}
& \left|\partial_{\xi_{0}}^{k} \partial_{\xi^{\prime}}^{k^{\prime}}\left(e_{0}\left(x_{3}+z\right)-e_{0}\left(x_{3}\right)\right)\right| \\
& \leq c \int_{0}^{x_{3}}\left|\partial_{\xi_{0}}^{k} \partial_{\xi^{\prime}}^{k^{\prime}}\left(e^{-\tau_{1}\left(x_{3}+z-y\right)}-e^{-\tau_{1}\left(x_{3}-y\right)}\right)\right| e^{-R e \tau_{2} y} d y \\
& \quad+c \int_{0}^{x_{3}}\left|e^{-\tau_{1}\left(x_{3}+z-y\right)}-e^{-\tau_{1}\left(x_{3}-y\right)}\right|\left|\partial_{\xi_{0}}^{k} \partial_{\xi^{\prime}}^{k^{\prime}} e^{-\tau_{2} y}\right| d y \\
& \quad+c \sum_{r+r^{\prime}=1}^{k+k^{\prime}-1} \int_{0}^{x_{3}}\left|\partial_{\xi_{0}}^{r} \partial_{\xi^{\prime}}^{r^{\prime}} e^{-\tau_{1}\left(x_{3}+z-y\right)}-\partial_{\xi_{0}}^{r} \partial_{\xi^{\prime}}^{r^{\prime}} e^{-\tau_{1}\left(x_{3}-y\right)} \| \partial_{\xi_{0}}^{r-k} \partial_{\xi^{\prime}}^{r^{\prime}-k^{\prime}} e^{-\tau_{2} y}\right| d y \\
& \quad+c \int_{x_{3}}^{x_{3}+z}\left|\partial_{\xi_{0}}^{k} \partial_{\xi^{\prime}}^{k^{\prime}} e^{-\tau_{1}\left(x_{3}+z-y\right)}\right| e^{-R e \tau_{2} y} d y \\
& \quad+c \int_{x_{3}}^{x_{3}+z} e^{-R e \tau_{1}\left(x_{3}+z-y\right)}\left|\partial_{\xi_{0}}^{k} \partial_{\xi^{\prime}}^{k^{\prime}} e^{-\tau_{2} y}\right| d y \\
& \quad+c \sum_{k+k^{\prime}-1}^{k+x_{3}+z} \int_{x_{3}}^{x_{3}+z}\left|\partial_{\xi_{0}}^{r} \partial_{\xi^{\prime}}^{r^{\prime}} e^{-\tau_{1}\left(x_{3}+z-y\right)} \| \partial_{\xi_{0}}^{r-k} \partial_{\xi^{\prime}}^{r^{\prime}-k^{\prime}} e^{-\tau_{2} y}\right| d y \equiv \sum_{i=1}^{6} I_{i} .
\end{aligned}
$$

First, using the formulas (3.15) and (3.12) we estimate

$$
\begin{aligned}
\left|I_{1}\right| \leq & c|\tau|^{-\left(2 k+k^{\prime}\right)} \int_{0}^{x_{3}} \mid\left[e^{-\tau_{1}\left(x_{3}+z-y\right)} e^{-\operatorname{Re} \tau_{2} y} \sum_{l=0}^{k+k^{\prime}-1}\left(x_{3}-y+z\right)^{l+1}\right. \\
& \left.\cdot|\tau|^{l+1}-e^{-\tau_{1}\left(x_{3}-y\right)} e^{-\operatorname{Re} \tau_{2} y} \sum_{l=0}^{k+k^{\prime}-1}\left(x_{3}-y\right)^{l+1}|\tau|^{l+1}\right] \mid d y \\
\leq & c|\tau|^{-\left(2 k+k^{\prime}\right)} \int_{0}^{x_{3}} e^{-\operatorname{Re} \tau_{1}\left(x_{3}-y\right)} e^{-\operatorname{Re} \tau_{1} z} e^{-\operatorname{Re} \tau_{2} y} \sum_{l=0}^{k+k^{\prime}-1}\left(\left(x_{3}-y\right)^{l}|z|\right. \\
& \left.+\left(x_{3}-y\right)^{l-1}|z|^{2}+\cdots+\left(x_{3}-y\right)^{2}|z|^{l-1}+\left(x_{3}-y\right)|z|^{l}\right)|\tau|^{l+1} d y \\
& +c|\tau|^{-\left(2 k+k^{\prime}\right)} \int_{0}^{x_{3}} e^{-\operatorname{Re} \tau_{1}\left(x_{3}-y\right)} e^{-\operatorname{Re} \tau_{2} y}\left|e^{-\operatorname{Re} \tau_{1} z}-1\right| \cdot \sum_{l=0}^{k+k^{\prime}-1}\left(x_{3}-y\right)^{l+1}|\tau|^{l+1} d y
\end{aligned}
$$


Hence

$$
\begin{aligned}
& \int_{0}^{\infty} \int_{0}^{\infty} \frac{\left|I_{1}\right|^{p}}{z^{1+p \varkappa}} d x_{3} d z \leq c|\tau|^{-\left(2 k+k^{\prime}\right)}\left[\int_{0}^{\infty} \frac{z^{p} e^{-p R e \tau_{1} z}}{z^{1+p \varkappa}} d z\right. \\
& \cdot \sum_{l=0}^{k+k^{\prime}-1} \int_{0}^{\infty}\left(\int_{0}^{x_{3}} e^{-\operatorname{Re} \tau_{1}\left(x_{3}-y\right)} e^{-\operatorname{Re} \tau_{2} y}\left(x_{3}-y\right)^{l}|\tau|^{l+1} d y\right)^{p} d x_{3} \\
& +\int_{0}^{\infty} \frac{z^{2 p} e^{-p R e \tau_{1} z}}{z^{1+p \varkappa}} d z \sum_{l=0}^{k+k^{\prime}-1} \int_{0}^{\infty}\left(\int_{0}^{x_{3}} e^{-\operatorname{Re} \tau_{1}\left(x_{3}-y\right)} e^{-\operatorname{Re} \tau_{2} y}\left(x_{3}-y\right)^{l-1}|\tau|^{l+1} d y\right)^{p} d x_{3} \\
& +\cdots+\int_{0}^{\infty} \frac{z^{p(l-1)} e^{-\operatorname{Re} \tau_{1} z}}{z^{1+p \varkappa}} d z \sum_{l=0}^{k+k^{\prime}-1} \int_{0}^{\infty}\left(\int_{0}^{x_{3}} e^{-\operatorname{Re} \tau_{1}\left(x_{3}-y\right)} e^{-\operatorname{Re} \tau_{2} y}\left(x_{3}-y\right)^{2}\right. \\
& \left.\cdot|\tau|^{l+1} d y\right)^{p} d x_{3}+\int_{0}^{\infty} \frac{z^{p l} e^{-p R e \tau_{1} z}}{z^{1+p \varkappa}} d z \sum_{l=0}^{k+k^{\prime}-1} \int_{0}^{\infty}\left(\int_{0}^{x_{3}} e^{-\operatorname{Re} \tau_{1}\left(x_{3}-y\right)}\right. \\
& \left.\left.\cdot e^{-\operatorname{Re} \tau_{2} y}\left(x_{3}-y\right)|\tau|^{l+1} d y\right)^{p} d x_{3}\right] \\
& +c|\tau|^{-\left(2 k+k^{\prime}\right)} \int_{0}^{\infty} \frac{1-e^{-p R e \tau_{1} z}}{z^{1+p \varkappa}} d z \sum_{l=0}^{k+k^{\prime}-1} \int_{0}^{\infty}\left(\int_{0}^{x_{3}} e^{-R e \tau_{1}\left(x_{3}-y\right)}\right. \\
& \left.\cdot e^{-\operatorname{Re\tau }_{2} y}\left(x_{3}-y\right)^{l+1}|\tau|^{l+1} d y\right)^{p} d x_{3} .
\end{aligned}
$$

Using as before the Young inequality we calculate

$$
\begin{aligned}
& \int_{0}^{\infty}\left(\int_{0}^{x_{3}} e^{-\operatorname{Re} \tau_{1}\left(x_{3}-y\right)} e^{-\operatorname{Re} \tau_{2} y}\left(x_{3}-y\right)^{r} d y\right)^{p} d x_{3} \\
& \leq c|\tau|^{-r p-p-1}, \quad r=1, \ldots, l,
\end{aligned}
$$

where we also used that $\left|\tau_{i}\right|<\frac{\sqrt{2}}{2} \operatorname{Re} \tau_{i}, i=1,2$ and (3.6)-(3.7).

Moreover,

$$
\int_{0}^{\infty} \frac{z^{p r} e^{-p R e \tau_{1} z}}{z^{1+p \varkappa}} d z \leq c|\tau|^{p \varkappa-p r}, \quad r=1, \ldots, l+1 .
$$

By (3.21)-(3.22) we get

$$
\int_{0}^{\infty} \int_{0}^{\infty} \frac{\left|I_{1}\right|^{p}}{z^{1+p \varkappa}} d x_{3} d z \leq c|\tau|^{-\left(2 k+k^{\prime}\right) p+p(\varkappa-1)-1} .
$$

Exactly in the same way we estimate

$$
\int_{0}^{\infty} \int_{0}^{\infty} \frac{\left|I_{2}\right|^{p}}{z^{1+p \varkappa}} d x_{3} d z
$$


Next, we have

$$
\begin{aligned}
\left|I_{3}\right| \leq & c|\tau|^{-\left(2 k+k^{\prime}\right)} \sum_{r+r^{\prime}=1}^{k+k^{\prime}-1} \int_{0}^{x_{3}} \mid e^{-\tau_{1}\left(x_{3}+z-y\right) .} \\
& \cdot \sum_{l=0}^{r+r^{\prime}-1}\left(x_{3}+z-y\right)^{l+1}|\tau|^{l+1} e^{-\operatorname{Re} \tau_{2} y} \sum_{m=0}^{k+k^{\prime}-r-r^{\prime}-1} y^{m+1}|\tau|^{m+1} \\
& -e^{-\tau_{1}\left(x_{3}-y\right)} \sum_{l=0}^{r+r^{\prime}-1}\left(x_{3}-y\right)^{l+1}|\tau|^{l+1} \sum_{m=0}^{k+k^{\prime}-r-r^{\prime}-1} y^{m+1}|\tau|^{m+1} \mid d y \\
\leq & c|\tau|^{-\left(2 k+k^{\prime}\right)} \sum_{m=1}^{k+k^{\prime}} \sum_{l=1}^{k+k^{\prime}-m} \int_{0}^{x_{3}} e^{-\operatorname{Re} \tau_{1}\left(x_{3}+z-y\right)} e^{-\operatorname{Re} \tau_{2} y} . \\
& \cdot\left[\left(x_{3}-y\right)^{m-1}|z|+\left(x_{3}-y\right)^{m-2}|z|^{2}+\cdots+\left(x_{3}-y\right)|z|^{m-1}+|z|^{m}\right] y^{l}|\tau|^{m+l} d y \\
& +c|\tau|^{-\left(2 k+k^{\prime}\right)} \sum_{m=1}^{k+k^{\prime}} \sum_{l=1}^{k^{\prime}-m} \int_{0}^{x_{3}} e^{-\operatorname{Re} \tau_{1}\left(x_{3}-y\right)} e^{-R e \tau_{2} y} \\
& \cdot\left|e^{-\operatorname{Re} \tau_{1} z}-1\right|\left(x_{3}-y\right)^{m} y^{l}|\tau|^{m+l} d y .
\end{aligned}
$$

Hence

$$
\begin{aligned}
& \int_{0}^{\infty} \int_{0}^{\infty} \frac{\left|I_{3}\right|^{p}}{z^{1+p \varkappa}} d x_{3} d z \leq c|\tau|^{-\left(2 k+k^{\prime}\right) p} \\
& \quad \sum_{m=1}^{k+k^{\prime}} \sum_{l=1}^{k+k^{\prime}-m}\left[\int _ { 0 } ^ { \infty } \frac { z ^ { p } e ^ { - p R e \tau _ { 1 } z } } { z ^ { 1 + p \varkappa } } d z \int _ { 0 } ^ { \infty } \left(\int_{0}^{x_{3}} e^{-\operatorname{Re} \tau_{1}\left(x_{3}-y\right)} e^{-\operatorname{Re} \tau_{2} y}\right.\right. \\
& \left.\cdot\left(x_{3}-y\right)^{m-1} y^{l}|\tau|^{m+l} d y\right)^{p} d x_{3} \\
& +\int_{0}^{\infty} \frac{z^{2 p} e^{-p R e \tau_{1} z}}{z^{1+p \varkappa}} d z \int_{0}^{\infty}\left(\int_{0}^{x_{3}} e^{-\operatorname{Re} \tau_{1}\left(x_{3}-y\right)} e^{-\operatorname{Re} \tau_{2} y}\right. \\
& \left.\quad \cdot\left(x_{3}-y\right)^{m-2} y^{l}|\tau|^{m+l} d y\right)^{p} d x_{3}+\cdots+\int_{0}^{\infty} \frac{z^{(m-1) p} e^{-p R e \tau_{1} z}}{z^{1+p \varkappa}} d z \\
& \left.\quad \int_{0}^{\infty}\left(\int_{0}^{x_{3}} e^{-\operatorname{Re} \tau_{1}\left(x_{3}-y\right)} e^{-\operatorname{Re} \tau_{2} y}\left(x_{3}-y\right) y^{l}|\tau|^{m+l} d y\right)^{p} d x_{3}\right] .
\end{aligned}
$$

Using in (3.23) estimates (3.21)-(3.22) we get

$$
\int_{0}^{\infty} \int_{0}^{\infty} \frac{\left|I_{3}\right|^{p}}{z^{1+p \varkappa}} d z d x_{3} \leq c|\tau|^{-\left(2 k+k^{\prime}\right) p+p(\varkappa-1)-1} .
$$

Next, we consider

$$
\begin{aligned}
& \int_{0}^{\infty} \int_{0}^{\infty} \frac{\left|I_{4}\right|^{p}}{z^{1+p \varkappa}} d z d x_{3} \leq c|\tau|^{-p\left(2 k+k^{\prime}\right)} \int_{0}^{\infty} \frac{d z}{z^{1+p \varkappa}} \\
& \left.\left.\quad \cdot \sum_{l=0}^{k+k^{\prime}-1} \int_{0}^{\infty}\left|\int_{x_{3}}^{x_{3}+z}\left(x_{3}+z-y\right)^{l+1}\right| \tau\right|^{l+1} e^{-\operatorname{Re\tau }_{1}\left(x_{3}+z-y\right)} \cdot e^{-\operatorname{Re} \tau_{2} y} d y\right|^{p} d x_{3} d z
\end{aligned}
$$




$$
\begin{aligned}
& \leq c|\tau|^{-p\left(2 k+k^{\prime}\right)} \int_{0}^{\infty} \frac{e^{-p R e \tau_{1} z}}{z^{1+p \varkappa}} d z \\
& \quad \cdot \sum_{l=0}^{k+k^{\prime}-1} \sup _{0 \leq y<\infty}\left(y^{l+1} e^{\frac{-p R e \tau_{2} y}{2}}\right)^{p}|\tau|^{p(l+1)} \int_{0}^{\infty}\left(\int_{x_{3}}^{x_{3}+z} e^{-p \frac{R e \tau_{2} y}{2}} d y\right) \\
& \quad\left(\int_{x_{3}}^{x_{3}+z} e^{-p R e \tau_{1}\left(x_{3}-y\right)} d y\right)^{p-1} d x_{3} \\
& \leq c|\tau|^{-p\left(2 k+k^{\prime}\right)-1} \int_{0}^{\infty} \frac{e^{-p R e \tau_{1} z}\left(1-e^{-p \frac{R e \tau_{2}}{2} z}\right)}{z^{1+p \varkappa}} d z \\
& \quad \cdot \int_{0}^{\infty} e^{-p \frac{\operatorname{Re\tau } \tau_{2}}{2} x_{3}}\left(\int_{x_{3}}^{x_{3}+z} e^{-p \operatorname{Re} \tau_{1}\left(x_{3}-y\right)} d y\right)^{p-1} d x_{3} \\
& \leq c|\tau|^{-p\left(2 k+k^{\prime}\right)+p(\varkappa-1)-1} .
\end{aligned}
$$

The integrals $\int_{0}^{\infty} \int_{0}^{\infty} \frac{\left|I_{i}\right|^{p}}{z^{1+p \varkappa}} d z d x_{3}, i=5,6$, we estimate in the same way.

Summarizing the above considerations we get

$$
\int_{0}^{\infty} \int_{0}^{\infty} \frac{\left|\partial_{\xi_{0}}^{k} \partial_{\xi^{\prime}}^{k^{\prime}}\left(e_{0}\left(x_{3}+z\right)-e_{0}\left(x_{3}\right)\right)\right|^{p}}{z^{1+p \varkappa}} d x_{3} d z \leq c|\tau|^{-p\left(2 k+k^{\prime}\right)+p \varkappa-p-1} .
$$

Hence, by the Leibniz formula

$$
\begin{aligned}
& \int_{0}^{\infty} \int_{0}^{\infty} \frac{\left|\partial_{\xi_{0}}^{k} \partial_{\xi^{\prime}}^{k^{\prime}}\left(A_{1}\left(x_{3}+z\right)-A_{1}\left(x_{3}\right)\right)\right|^{p}}{z^{1+p \varkappa}} d x_{3} d z \\
& \leq c \sum_{r=0}^{k} \sum_{r^{\prime}=0}^{k^{\prime}} \int_{0}^{\infty} \int_{0}^{\infty}|\tau|^{p\left[j-(k-r)-2\left(k^{\prime}-r^{\prime}\right)\right]} \cdot \frac{\left|\partial_{\xi_{0}}^{r} \partial_{\xi^{\prime}}^{r^{\prime}}\left(e_{0}\left(x_{3}+z\right)-e_{0}\left(x_{3}\right)\right)\right|^{p}}{z^{1+p \varkappa}} d x_{3} d z \\
& \leq c|\tau|^{p j-p\left(2 k+k^{\prime}\right)-p-1} .
\end{aligned}
$$

Inequalities (3.20) and (3.24) yield (3.19).

This completes the proof.

Now, we can prove the main result of this section.

TheOREM 3.4. Let $p, q \in(1, \infty), \sigma \in \mathbb{R}_{+}, \sigma>1+\frac{1}{p}, b_{k} \in B_{p, q, \gamma}^{\left(\sigma-1-\frac{1}{p}\right) / 2, \sigma-1-\frac{1}{p}}\left(\mathbb{R}_{+} \times \mathbb{R}^{2}\right)$, $k=1,2, b_{3} \in B_{p, q, \gamma}^{\left(\sigma-\frac{1}{p}\right) / 2, \sigma-\frac{1}{p}}\left(\mathbb{R}_{+} \times \mathbb{R}^{2}\right)$. Then there exists a unique solution to problem (3.1) such that $u \in B_{p, q, \gamma}^{\frac{\sigma}{2}, \sigma}\left(\mathbb{R}_{+} \times \mathbb{R}^{3}\right)$ and

$$
\begin{aligned}
& \|u\|_{B_{p, q, \gamma}^{\frac{\sigma}{2}, \sigma}\left(\mathbb{R}_{+} \times \mathbb{R}_{+}^{3}\right)} \\
& \quad \leq c\left(\sum_{k=1}^{2}\left\|b_{k}\right\|_{B_{p, q, \gamma}^{(\sigma-1-1 / p) / 2, \sigma-1-\frac{1}{p}}\left(\mathbb{R}_{+} \times \mathbb{R}^{2}\right)}+\left\|b_{3}\right\|_{B_{p, q, \gamma}^{\left(\sigma-\frac{1}{p}\right) / 2, \sigma-\frac{1}{p}}} \mathbb{R}_{\left(\mathbb{R}_{+} \times \mathbb{R}^{2}\right)}\right) .
\end{aligned}
$$

The proof of Theorem 3.4 follows from the lemmas below. 
Lemma 3.5. Let the assumptions of Theorem 3.4 be satisfied. Then

$$
\begin{aligned}
& \|u\|_{1, B_{p, q, \gamma}^{\frac{\sigma}{2}, \sigma}\left(\mathbb{R}_{+} \times \mathbb{R}_{+}^{3}\right)} \leq c\left(\sum_{k=1}^{2}\left\|b_{k}\right\|_{B_{p, q, \gamma}^{\left(\sigma-1-\frac{1}{p}\right) / 2, \sigma-1-\frac{1}{p}}\left(\mathbb{R}_{+} \times \mathbb{R}^{2}\right)}\right) \\
& \left.\quad+\left\|b_{3}\right\|_{B_{p, q, \gamma}^{\left(\sigma-\frac{1}{p}\right) / 2, \sigma-\frac{1}{p}}\left(\mathbb{R}_{+} \times \mathbb{R}^{2}\right)}\right),
\end{aligned}
$$

where $u=\left(u_{1}, u_{2}, u_{3}\right)$ and

$$
\begin{aligned}
& \left\|u_{m}\right\|_{1, B_{p, q, \gamma}^{\frac{\sigma}{2}, \sigma}\left(\mathbb{R}_{+} \times \mathbb{R}_{+}^{3}\right)}=\left[\sum_{k=0}^{\infty}\left(\sum_{j \leq[\sigma]} \int_{\mathbb{R} \times \mathbb{R}_{+}^{3}}\left|e^{-\gamma t} 2^{(\sigma-j) k} F_{2}^{-1} \phi_{k} F_{2} \partial_{x_{3}}^{j} u_{m}\right|^{p} d t d x\right)^{\frac{q}{p}}\right]^{1 / q}, \\
& m=1,2,3 .
\end{aligned}
$$

Proof. Using (3.5) we have

$$
\begin{aligned}
& \left\|u_{m}\right\|_{1, B_{p, q, \gamma}^{\frac{\sigma}{2}, \sigma}\left(\mathbb{R}_{+} \times \mathbb{R}_{+}^{3}\right)}=\left[\sum _ { k = 0 } ^ { \infty } \left(\sum_{j \leq[\sigma]} \int_{\mathbb{R} \times \mathbb{R}_{+}^{3}} \mid e^{-\varkappa} 2^{(\sigma-j) k} F_{2}^{-1} \phi_{k} \sum_{r=1}^{3}\left(g_{m r} \partial_{x_{3}}^{j} e_{0}\right.\right.\right. \\
& \left.\left.\left.+h_{m r} \partial_{x_{3}}^{j} e_{1}\right)\left.\hat{b}_{r}\right|^{p} d t d x\right)^{\frac{q}{p}}\right]^{\frac{1}{q}} \leq c\left(\sum_{k=0}^{\infty} \sum_{j \leq[\sigma]} \sum_{r=1}^{3} I_{1 k j r}^{q}\right)^{1 / q}
\end{aligned}
$$

where

$$
I_{1 k j r}=\left(\int_{\mathbb{R} \times \mathbb{R}_{+}^{3}}\left|e^{-\gamma t} 2^{(\sigma-j) k} F_{2}^{-1} \phi_{k}\left(g_{m r} \partial_{x_{3}}^{j} e_{0}+h_{m r} \partial_{x_{3}}^{j} e_{1}\right) F_{2} b_{r}\right|^{p} d t d x\right)^{1 / p} .
$$

Introduce the family of functions $\left\{\psi_{j}(\bar{\xi})\right\}, \bar{\xi}=\left(\xi_{0}, \xi^{\prime}\right), \xi^{\prime}=\left(\xi_{1}, \xi_{2}\right)$ such that supp $\psi_{0}$ $\subset\left\{\bar{\xi}:|\bar{\xi}|_{a} \leq 4\right\}$, supp $\psi_{j} \subset\left\{\bar{\xi}: 2^{j-2} \leq|\bar{\xi}|_{a} \leq 2^{j+2}\right\}$ and $\psi_{j}(\bar{\xi})=1$ for $\bar{\xi} \in \operatorname{supp} \phi_{j}$. Then

$$
\begin{aligned}
I_{1 k j r}= & \left(\int_{\mathbb{R} \times \mathbb{R}_{+}^{3}}\left|\sum_{l=0}^{\infty} 2^{(\sigma-j) k} e^{-\gamma t} F_{2}^{-1} \psi_{l}\left(g_{m r} \partial_{x_{3}}^{j} e_{0}+h_{m r} \partial_{x_{3}}^{j} e_{1}\right) \phi_{k} \phi_{l} F_{2} b_{r}\right|^{p} d t d x\right)^{1 / p} \\
= & \left(\int_{\mathbb{R}^{3} \times \mathbb{R}_{+}} \mid \sum_{l=0}^{\infty} 2^{(\sigma-j) k} e^{-\gamma x_{0}}\left(F_{2}^{-1} \psi_{l}\left(g_{m r} \partial_{x_{3}}^{j} e_{0}+h_{m r} \partial_{x_{3}}^{j} e_{1}\right)\right.\right. \\
& \left.\left.\cdot F_{2} F_{2}^{-1} \phi_{k} F_{2} F_{2}^{-1} \phi_{l} F_{2} b_{r}\right)\left.\left(\bar{x}, x_{3}\right)\right|^{p} d \bar{x} d x_{3}\right)^{1 / p},
\end{aligned}
$$

where $\bar{x}=\left(t, x^{\prime}\right)=\left(x_{0}, x^{\prime}\right), x^{\prime}=\left(x_{1}, x_{2}\right) \in \mathbb{R}^{2}$. Continuing, we can rewrite $I_{1 k j r}$ as

$$
\begin{aligned}
I_{1 k j r}= & \left(\int_{\mathbb{R}^{3} \times \mathbb{R}_{+}} \mid \sum_{l=0}^{\infty} 2^{(\sigma-j) k} e^{-\gamma x_{0}}\left[F _ { 2 } ^ { - 1 } \psi _ { l } \left(g_{m r} \partial_{x_{3}}^{j} e_{0}\right.\right.\right. \\
& \left.\left.\left.+h_{m r} \partial_{x_{3}}^{j} e_{1}\right) F_{2} F_{2}^{-1} \phi_{k} * F_{2}^{-1} \phi_{l} F_{2} b_{r}\right]\left.\left(\bar{x}, x_{3}\right)\right|^{p} d \bar{x} d x_{3}\right)^{1 / p} \\
= & \left(\int_{\mathbb{R}^{3} \times \mathbb{R}_{+}} \mid \sum_{l=0}^{\infty} 2^{(\sigma-j) k} e^{-\gamma x_{0}} \int_{\mathbb{R}^{3}} d \bar{y}\left[F_{2}^{-1} \psi_{l}\left(g_{m r} \partial_{x_{3}}^{j} e_{0}+h_{m r} \partial_{x_{3}}^{j} e_{1}\right)\right.\right. \\
& \left.\left.\cdot F_{2} F_{2}^{-1} \phi_{k}\right]\left.\left(\bar{y}, x_{n}\right)\left(F_{2}^{-1} \phi_{l} F_{2} b_{r}\right)(\bar{x}-\bar{y})\right|^{p} d \bar{x} d x_{3}\right)^{1 / p} \cdot
\end{aligned}
$$


Similarly as in [27] (see the proof of Lemma 3.2) we shall use in the sequel the formulas:

$$
\begin{aligned}
& F_{2}^{-1}\left[\psi_{l}\left(g_{m r} \partial_{x_{3}}^{j} e_{0}+h_{m r} \partial_{x_{3}}^{j} e_{1}\right) F_{2} F_{2}^{-1} \phi_{k}\right]\left(\bar{y}, x_{3}\right) \\
& =\left[F_{2}^{-1} \psi_{l}\left(g_{m r} \partial_{x_{3}}^{j} e_{0}+h_{m r} \partial_{x_{3}}^{j} e_{1}\right) * F_{2}^{-1} \phi_{k}\right]\left(\bar{y}, x_{3}\right)
\end{aligned}
$$

and

$$
\left[F_{2}^{-1}\left(f\left(2^{2 l} \cdot, 2^{l} \cdot\right)\right) * F_{2}^{-1}\left(g\left(2^{2 l} \cdot, 2^{l} \cdot\right)\right)\right](\bar{y})=2^{-4 l}\left(F_{2}^{-1} f * F_{2}^{-1} g\right)\left(2^{-l} \tilde{y}\right),
$$

where $f=\psi_{l}\left(g_{m r} \partial_{x_{3}}^{j} e_{0}+h_{m r} \partial_{x_{3}}^{j} e_{1}\right), g=\phi_{k}, \tilde{y}=\left(2^{-l} y_{0}, y^{\prime}\right), y^{\prime}=\left(y_{1}, y_{2}\right)$.

Moreover, we used in (3.27) the notation

$$
h\left(\xi_{0}, \xi^{\prime}\right)=\tilde{h}\left(\gamma+i \xi_{0}, \xi^{\prime}\right), \quad h \in\{f, g\}
$$

and the relation

$$
\left(F_{2}^{-1} \tilde{h}\left(\gamma+i 2^{2 l} \xi_{0}, 2^{l} \xi^{\prime}\right)\right)\left(y_{0}, y^{\prime}\right)=2^{-4 l}\left(F_{2}^{-1} \tilde{h}\left(\gamma+i \xi_{0}, \xi^{\prime}\right)\right)\left(2^{-l} \tilde{y}\right)
$$

Applying (3.26), the expression $I_{1 k j r}$ takes the form

$$
\begin{aligned}
I_{1 k j r}= & {\left[\int_{\mathbb{R}^{3} \times \mathbb{R}_{+}} \mid \sum_{l=0}^{\infty} 2^{(\sigma-j) k} e^{-\gamma x_{0}} 2^{-4 l} \int_{\mathbb{R}^{3}} d \bar{w}\left[F _ { 2 } ^ { - 1 } \psi _ { l } \left(g_{m r} \partial_{x_{3}}^{j} e_{0}\right.\right.\right.} \\
& \left.\left.\left.+h_{m r} \partial_{x_{3}}^{j} e_{1}\right) * F_{2}^{-1} \phi_{k}\right]\left.\left(2^{-l} \tilde{w}, x_{n}\right)\left(F_{2}^{-1} \phi_{l} F_{2} b_{r}\right)\left(\bar{x}-2^{-l} \tilde{w}\right)\right|^{p} d \bar{x} d x_{n}\right]^{1 / p},
\end{aligned}
$$

where we used the change of variables $y_{i}=2^{-l} w_{i}, i=1,2, y_{0}=2^{-2 l} w_{0}$ and the notation $\tilde{w}=\left(2^{-l} w_{0}, w^{\prime}\right), w^{\prime}=\left(w_{1}, w_{2}\right), \bar{w}=\left(w^{\prime}, w_{0}\right)$. Then (3.27) yields

$$
\begin{aligned}
I_{1 k j r}= & \left\{\int_{\mathbb{R}^{3} \times \mathbb{R}_{+}} d \bar{x} d x_{3} \mid \sum_{l=0}^{\infty} 2^{(\sigma-j) k} e^{-\gamma x_{0}} \int_{\mathbb{R}^{n}} d \bar{w}\left[F _ { 2 } ^ { - 1 } \left(\psi _ { l } \left(g_{m r} \partial_{x_{3}}^{j} e_{0}\right.\right.\right.\right. \\
& \left.\left.\left.\left.+h_{m r} \partial_{x_{3}}^{j} e_{1}\right)\left(2^{2 l} \cdot, 2^{l} \cdot\right)\right) * F_{2}^{-1}\left(\phi_{k}\left(2^{2 l} \cdot, 2^{l} \cdot\right)\right)\right]\left.\left(\bar{w}, x_{3}\right)\left(F_{2}^{-1} \phi_{l} F_{2} b_{r}\right)(\bar{x}-\bar{w})\right|^{p}\right\}^{1 / p} \\
= & \left\{\int_{\mathbb{R}_{+}} d x_{3} \int_{\mathbb{R}^{3}} d \bar{x} \mid \sum_{l=0}^{\infty} 2^{(\sigma-j) k} \int_{\mathbb{R}^{3}} d \bar{w} e^{-\gamma w_{0}}\left[F_{2}^{-1}\left(\psi_{l}\left(g_{m r} \partial_{x_{3}}^{j} e_{0}+h_{m r} \partial_{x_{3}}^{j} e_{1}\right)\left(2^{2 l} \cdot, 2^{l} \cdot\right)\right)\right.\right. \\
& \left.\left.* F_{2}^{-1}\left(\phi_{k}\left(2^{2 l} \cdot, 2^{l} \cdot\right)\right)\right]\left.\left(\bar{w}, x_{3}\right) e^{-\gamma\left(x_{0}-w_{0}\right)}\left(F_{2}^{-1} \phi_{l} F_{2} b_{r}\right)(\bar{x}-\bar{w})\right|^{p}\right\}^{1 / p} .
\end{aligned}
$$

Next, the Minkowski inequality with respect to $\bar{x}$ gives

$$
\begin{aligned}
I_{1 k j r} \leq & {\left[\int_{\mathbb{R}_{+}} d x_{3} \mid \sum_{l=0}^{\infty} 2^{(\sigma-j) k} \int_{\mathbb{R}^{3}} d \bar{w} e^{-\gamma w_{0}}\left[F _ { 2 } ^ { - 1 } \left(\psi _ { l } \left(g_{m r} \partial_{x_{3}}^{j} e_{0}\right.\right.\right.\right.} \\
& \left.\left.\left.+h_{m r} \partial_{x_{3}}^{j} e_{1}\right)\left(2^{2 l} \cdot, 2^{l} \cdot\right)\right) * F_{2}^{-1}\left(\phi_{k}\left(2^{2 l} \cdot, 2^{l} \cdot\right)\right)\right]\left(\bar{w}, x_{3}\right) \\
& \left.\left.\cdot\left(\int_{\mathbb{R}^{3}} d \bar{x}\left|e^{-\gamma\left(x_{0}-w_{0}\right)}\left(F_{2}^{-1} \phi_{l} F_{2} b_{r}\right)(\bar{x}-\bar{w})\right|^{p}\right)^{1 / p}\right|^{p}\right]^{1 / p} \equiv I_{1 k j r}^{1} .
\end{aligned}
$$


The change of variables $\bar{z}=\bar{x}-\bar{w}$ in the integral with respect to $\bar{x}$ implies

$$
\begin{aligned}
I_{1 k j r}^{1}= & \left\{\int_{\mathbb{R}_{+}} d x_{3} \mid \sum_{l=0}^{\infty} 2^{(\sigma-j) k} \int_{\mathbb{R}^{3}} d \bar{w} e^{-\gamma w_{0}}\left[F _ { 2 } ^ { - 1 } \left(\psi _ { l } \left(g_{m r} \partial_{x_{3}}^{j} e_{0}\right.\right.\right.\right. \\
& \left.\left.\left.+h_{m r} \partial_{x_{3}}^{j} e_{1}\right)\left(2^{2 l} \cdot, 2^{l} \cdot\right)\right) * F_{2}^{-1}\left(\phi_{k}\left(2^{2 l} \cdot, 2^{l} \cdot\right)\right)\right]\left(\bar{w}, x_{3}\right) \\
& \left.\left.\cdot\left(\int_{\mathbb{R}^{3}} d \bar{z}\left|e^{-\gamma z_{0}}\left(F_{1}^{-1} \phi_{l} F_{1} b_{r}\right)(\bar{z})\right|^{p}\right)^{1 / p}\right|^{p}\right\}^{1 / p},
\end{aligned}
$$

where $\bar{z}=\left(z_{0}, z^{\prime}\right)$, and we used the fact that $F_{2} b_{r}=F_{1} b_{r}$.

Next, using the Hölder inequality in the integral with respect to $\bar{w}$ and replacing $\bar{w}$ by $\bar{y}$ and $\bar{z}$ by $\bar{w}$ we obtain

$$
\begin{aligned}
I_{1 k j r}^{1} \leq & \left\{\int_{\mathbb{R}_{+}} d x_{3} \mid \sum_{l=0}^{\infty} 2^{(\sigma-j) k}\left(\int_{\mathbb{R}^{3}} d \bar{y} \frac{1}{\left(1+|\bar{y}|_{a}\right)^{d}}\right)^{1 / 2}\right. \\
& \cdot\left(\int_{\mathbb{R}^{3}} d \bar{y} \mid e^{-\gamma y_{0}}\left[F_{2}^{-1}\left(\psi_{l}\left(g_{m r} \partial_{x_{3}}^{j} e_{0}+h_{m r} \partial_{x_{3}}^{j} e_{1}\right)\left(2^{2 l} \cdot, 2^{l} \cdot\right)\right) *\right.\right. \\
& \left.\left.\left.* F_{2}^{-1}\left(\phi_{k}\left(2^{2 l} \cdot, 2^{l} \cdot\right)\right)\right]\left.\left(\bar{y}, x_{3}\right) \cdot\left(1+|\bar{y}|_{a}\right)^{\frac{d}{2}}\right|^{2}\right)\left.^{1 / 2}\right|^{p}\right\}^{1 / p} \\
& \cdot\left(\int_{\mathbb{R}^{3}} d \bar{x}\left|e^{-\gamma x_{0}}\left(F_{2}^{-1} \phi_{l} F_{2} b_{r}\right)(\bar{x})\right|^{p}\right)^{1 / p} \equiv I_{1 k j r}^{2} .
\end{aligned}
$$

Assuming that $d>3$ we get that

$$
\left(\int_{\mathbb{R}^{3}} d \bar{y} \frac{1}{\left(1+|\bar{y}|_{a}\right)^{d}}\right)^{1 / 2} \leq c .
$$

Hence, in view of the Parseval identity we have

$$
\begin{aligned}
I_{1 k j r}^{3}= & c\left\{\int_{\mathbb{R}_{+}} d x_{3} \mid \sum_{l=0}^{\infty} 2^{(\sigma-j)(k-l)} 2^{(\sigma-j) l} \| \psi_{l}\left(g_{m r} \partial_{x_{3}}^{j} e_{0}\right.\right. \\
& \left.\left.\left.+h_{m r} \partial_{x_{3}}^{j} e_{1}\right)\right)\left(2^{2 l} \cdot, 2^{l} \cdot, x_{3}\right) \phi_{k}\left(2^{2 l} \cdot, 2^{l} \cdot\right) \|\left._{W_{2}^{\frac{d}{2}, d}\left(\mathbb{R}^{3}\right)}\right|^{p}\right\}^{1 / p} \\
& \cdot\left(\int_{\mathbb{R}^{3}} d \bar{x}\left|e^{-\gamma x_{0}}\left(F_{1}^{-1} \phi_{l} F_{1} b_{r}\right)(\bar{x})\right|^{p}\right)^{1 / p} \\
\leq & c \sum_{l=0}^{\infty} 2^{(\sigma-j)(k-l)} 2^{(\sigma-j) l}\left(\int_{\mathbb{R}_{+}} \| \psi_{l}\left(g_{m r} \partial_{x_{3}}^{j} e_{0}\right.\right. \\
& \left.\left.\left.+h_{m r} \partial_{x_{3}}^{j} e_{1}\right)\right)\left(2^{2 l} \cdot, 2^{l} \cdot, x_{3}\right) \phi_{k}\left(2^{2 l} \cdot, 2^{l} \cdot\right) \|_{W_{2}^{\frac{d}{2}, d}\left(\mathbb{R}^{3}\right)}\right)^{1 / p} \\
& \cdot\left(\int_{\mathbb{R}^{3}} d \bar{x}\left|e^{-\gamma x_{0}}\left(F_{1}^{-1} \phi_{k} F_{1} b_{r}\right)(\bar{x})\right|^{p}\right)^{1 / p} \equiv I_{1 k j r}^{4} .
\end{aligned}
$$

Using inequality (3.29) (see Lemma 3.5 below) we get

$$
I_{1 k j r} \leq c \sum_{l=0}^{\infty} 2^{(\delta-j+d+4-L)|l-k|} 2^{\left(\delta-\frac{1}{p}-c_{r}\right) l}\left\|e^{-\gamma x_{0}} F_{1}^{-1} \phi_{l} F_{1} b_{r}\right\|_{L_{p}\left(\mathbb{R}^{3}\right)}
$$


where $c_{r}=1$ for $r=1,2, c_{r}=0$ for $r=3$. Therefore, by the Hölder inequality

$$
\begin{aligned}
& \left\|u_{m}\right\|_{1, B_{p, q, \gamma}^{\frac{\sigma}{2}, \sigma}\left(\mathbb{R}_{+} \times \mathbb{R}_{+}^{3}\right)} \leq c\left\{\sum_{k=0}^{\infty} \sum_{r=1}^{3} \mid \sum_{l=0}^{\infty} 2^{(\delta-L)|l-k|} 2^{\left(\sigma-\frac{1}{p}+c_{r}\right) l}\right. \\
& \left.\left.\cdot\left\|e^{-\gamma x_{0}} F_{1}^{-1} \phi_{l} F_{1} b_{r}\right\|_{L_{p}\left(\mathbb{R}^{3}\right)}\right|^{q}\right\}^{1 / q} \\
& \leq c\left(\sum_{k=0}^{\infty} \sum_{r=1}^{3} \sum_{l=0}^{\infty} 2^{(\delta+\varepsilon-L)|l-k| q} 2^{\left(\sigma-\frac{1}{p}-c_{r}\right) l q}\left\|e^{-\gamma t} F_{2}^{-1} \phi_{l} F_{2} b_{r}\right\|_{L_{p}\left(\mathbb{R}^{3}\right)}^{q}\right)^{1 / q},
\end{aligned}
$$

where $\delta=\sigma+d+4, t=x_{0}$ and $\varepsilon>0$ is arbitrarily small. Assuming that $L>\delta+\varepsilon$ we obtain (3.25).

This ends the proof.

To prove (3.28) we need

Lemma 3.6. Let $d$ be an even number such that $d>3$. Then

$$
\begin{aligned}
J_{m r} \equiv & \left(\int_{\mathbb{R}_{+}} d x_{3} \|\left[\psi_{l}\left(g_{m r} \partial_{x_{3}}^{j} e_{0}+h_{m r} \partial_{x_{3}}^{j} e_{1}\right)\right]\left(2^{2 l} \cdot, 2^{l} \cdot, x_{3}\right)\right. \\
& \left.\left.\cdot \phi_{k}\left(2^{2 l} \cdot, 2^{l} \cdot\right) \|_{W_{2}^{\frac{d}{2}, d}}^{p}\right)_{\left(\mathbb{R} \times \mathbb{R}^{2}\right)}\right)^{1 / p} \leq c 2^{l\left(j-\frac{1}{p}-c_{r}\right)} 2^{(d+4-L)|l-k|},
\end{aligned}
$$

where for $l=0$ the constant $c$ depends on $\gamma, c_{r}=1$ for $r=1,2 ; c_{r}=0$ for $r=3$; $m=1,2,3 ; L>0$ can be chosen sufficiently large.

Proof. $J_{m r}$ can be written as

$$
\begin{aligned}
J_{m r}= & \left(\int_{\mathbb{R}_{+}} d x_{3} \sum_{\sum_{i=1}^{4}} \sum_{\left(s_{i}^{\prime}+2 s_{i}\right) \leq d} \|\left(\partial_{\xi^{\prime}}^{s_{1}^{\prime}} \partial_{\xi_{0}}^{s_{1}} \psi_{l}\right)\left(2^{2 l} \cdot, 2^{l} \cdot\right)\left(\partial_{\xi^{\prime}}^{s_{2}^{\prime}} \partial_{\xi_{0}}^{s_{2}} g_{m r} \partial_{\xi^{\prime}}^{s_{3}^{\prime}} \partial_{\xi_{0}}^{s_{3}} \partial_{x_{3}}^{j} e_{0}\right.\right. \\
& \left.\left.+\partial_{\xi^{\prime}}^{s_{2}^{\prime}} \partial_{\xi_{0}}^{s_{2}} h_{m r} \partial_{\xi^{\prime}}^{s_{3}^{\prime}} \partial_{\xi_{0}}^{s_{3}} \partial_{x_{3}}^{j} e_{1}\right)\left(2^{2 l} \cdot, 2^{l} \cdot, x_{3}\right)\left(\partial_{\xi^{\prime}}^{s_{4}^{\prime}} \partial_{\xi_{0}}^{s_{4}} \phi_{k}\right)\left(2^{2 l} \cdot, 2^{l} \cdot\right) \|_{L_{2}\left(\mathbb{R}^{3}\right)}^{p}\right)^{1 / p} .
\end{aligned}
$$

Applying the Minkowski inequality we get

$$
\begin{aligned}
J_{m r} \leq & c \sum_{\sum_{i=1}^{4}\left(s_{i}^{\prime}+2 s_{i}\right) \leq d} \|\left(\partial_{\xi^{\prime}}^{s_{1}^{\prime}} \partial_{\xi_{0}}^{s_{1}} \psi_{l}\right)\left(2^{2 l} \cdot, 2^{l} \cdot\right) \partial_{\xi^{\prime}}^{s_{2}^{\prime}} \partial_{\xi_{0}}^{s_{2}} g_{m r}\left(2^{2 l} \cdot, 2^{l} \cdot\right) \\
& \cdot\left\|\partial_{\xi^{\prime}}^{s_{3}^{\prime}} \partial_{\xi_{0}}^{s_{3}} \partial_{x_{3}}^{j} e_{0}\right\|_{L_{p}\left(\mathbb{R}_{+}\right)}\left(2^{2 l} \cdot, 2^{l} \cdot\right)\left(\partial_{\xi^{\prime}}^{s_{4}^{\prime}} \partial_{\xi_{0}}^{s_{4}} \phi_{k}\right)\left(2^{2 l} \cdot, 2^{l} \cdot\right) \|_{L_{2}\left(\mathbb{R}^{3}\right)} \\
& +\left\|\left(\partial_{\xi^{\prime}}^{s_{1}^{\prime}} \partial_{\xi_{0}}^{s_{1}} \psi_{l}\right)\left(2^{2 l} \cdot, 2^{l} \cdot\right) \partial_{\xi^{\prime}}^{s_{2}^{\prime}} \partial_{\xi_{0}}^{s_{2}} h_{m r}\left(2^{2 l} \cdot, 2^{l} \cdot\right)\right\| \partial_{\xi^{\prime}}^{s_{3}^{\prime}} \partial_{\xi_{0}}^{s_{3}} \partial_{x_{3}}^{j} e_{1} \|_{L_{p}\left(\mathbb{R}_{+}\right)}\left(2^{2 l} \cdot, 2^{l} \cdot\right) \\
& \left.\cdot\left(\partial_{\xi^{\prime}}^{s_{4}^{\prime}} \partial_{\xi_{0}}^{s_{4}} \phi_{k}\right)\left(2^{2 l} \cdot, 2^{l} \cdot\right) \|_{L_{2}\left(\mathbb{R}^{3}\right)}\right) \equiv J_{1 m r} .
\end{aligned}
$$

From the properties of $\psi_{l}$ it follows that

$$
\begin{array}{ll}
\operatorname{supp} \psi_{l}\left(2^{2 l} \cdot, 2^{l} \cdot\right) \subset\left\{\bar{\xi}:|\bar{\xi}|_{a} \leq 4\right\} & \text { for } l=0, \\
\operatorname{supp} \psi_{l}\left(2^{2 l} \cdot, 2^{l} \cdot\right) \subset\left\{\bar{\xi}: 1 / 4 \leq|\bar{\xi}|_{a} \leq 4\right\} & \text { for } l \neq 0
\end{array}
$$

and

$$
\left|\partial_{\xi^{\prime}}^{s_{1}^{\prime}} \partial_{\xi_{0}}^{s_{1}} \psi_{l}\left(2^{2 l} \cdot, 2^{l} \cdot\right)\right| \leq c \quad \text { for } \bar{\xi} \in A,
$$


where

$$
\begin{array}{ll}
A=\left\{\bar{\xi}:|\bar{\xi}|_{a} \leq 4\right\} & \text { for } l=0 \text { or } \\
A=\left\{\bar{\xi}: 1 / 4 \leq|\bar{\xi}|_{a} \leq 4\right\} & \text { for } l \neq 0 .
\end{array}
$$

Therefore, we obtain

$$
\begin{aligned}
J_{1 m r} \leq & c \sum_{\sum_{i=1}^{4}\left(s_{i}^{\prime}+2 s_{i}\right) \leq d}\left(\left\|\partial_{\xi^{\prime}}^{s_{2}^{\prime}} \partial_{\xi_{0}}^{s_{2}} g_{m r}\left(2^{2 l} \cdot, 2^{l} \cdot\right)\right\| \partial_{\xi^{\prime}}^{s_{3}^{\prime}} \partial_{\xi_{0}}^{s_{3}} \partial_{x_{3}}^{j} e_{0} \|_{L_{p}\left(\mathbb{R}_{+}\right)}\left(2^{2 l} \cdot, 2^{l} \cdot\right)\right. \\
& \cdot\left(\partial_{\xi^{\prime}}^{s_{4}^{\prime}} \partial_{\xi_{0}}^{s_{4}} \phi_{k}\right)\left(2^{2 l} \cdot, 2^{l} \cdot\right)\left\|_{L_{2}(A)}+\right\| \partial_{\xi^{\prime}}^{s_{2}^{\prime}} \partial_{\xi_{0}}^{s_{2}} h_{m r}\left(2^{2 l} \cdot, 2^{l} \cdot\right) \\
& \left.\cdot\left\|\partial_{\xi^{\prime}}^{s_{3}^{\prime}} \partial_{\xi_{0}}^{s_{3}} \partial_{x_{3}}^{j} e_{1}\right\|_{L_{p}\left(\mathbb{R}_{+}\right)}\left(2^{2 l} \cdot, 2^{l} \cdot\right)\left(\partial_{\xi^{\prime}}^{s_{4}^{\prime}} \partial_{\xi_{0}}^{s_{4}} \phi_{k}\right)\left(2^{2 l} \cdot, 2^{l} \cdot\right) \|_{L_{2}(A)}\right) .
\end{aligned}
$$

By Lemma 3.1

$$
\begin{gathered}
\left|\partial_{\xi^{\prime}}^{s_{2}^{\prime}} \partial_{\xi_{0}}^{s_{2}} g_{m r}\left(2^{2 l} \cdot, 2^{l} \cdot\right)\right| \leq \frac{c 2^{l\left(s_{2}^{\prime}+2 s_{2}\right)}}{|\tau|^{s_{2}^{\prime}+2 s_{2}}} \text { for } r=1,2, \\
\left|\partial_{\xi^{\prime}}^{s_{2}^{\prime}} \partial_{\xi_{0}}^{s_{2}} g_{m 3}\left(2^{2 l} \cdot, 2^{l} \cdot\right)\right| \leq \frac{c 2^{l\left(s_{2}^{\prime}+2 s_{2}\right)}}{|\tau|^{s_{2}^{\prime}+2 s_{2}-1}}, \\
\left|\partial_{\xi^{\prime}}^{s_{2}^{\prime}} \partial_{\xi_{0}}^{s_{2}} h_{m r}\right| \leq \frac{c 2^{l\left(s_{2}^{\prime}+2 s_{2}\right)}}{|\tau|^{s_{2}^{\prime}+2 s_{2}+1}} \text { for } r=1,2, \\
\left|\partial_{\xi^{\prime}}^{s_{2}^{\prime}} \partial_{\xi_{0}}^{s_{2}} h_{m 3}\right| \leq \frac{c 2^{l\left(s_{2}^{\prime}+2 s_{2}\right)}}{|\tau|^{s_{2}^{\prime}+2 s_{2}}}
\end{gathered}
$$

where now $\tau^{2}=\gamma+2^{2 l} \xi^{\prime 2}+2^{2 l} \xi_{0} i$. Lemma 3.2 yields the estimates

$$
\left\|\partial_{x_{3}}^{j} \partial_{\xi^{\prime}}^{s_{3}^{\prime}} \partial_{\xi_{0}}^{s_{3}} e_{0}\right\|_{L_{p}\left(\mathbb{R}_{+}\right)}\left(2^{2 l} \cdot, 2^{l}\right) \leq \frac{c 2^{l\left(s_{3}^{\prime}+2 s_{3}\right)}}{|\tau|^{s_{3}^{\prime}+2 s_{3}}}|\tau|^{j-\frac{1}{p}-1}
$$

and

$$
\left\|\partial_{x_{3}}^{j} \partial_{\xi^{\prime}}^{s_{3}^{\prime}} \partial_{\xi_{0}}^{s_{3}} e_{1}\right\|_{L_{p}\left(\mathbb{R}_{+}\right)}\left(2^{2 l} \cdot, 2^{l} \cdot\right) \leq \frac{c 2^{l\left(s_{3}^{\prime}+2 s_{3}\right)}}{|\tau|^{s_{3}^{\prime}+2 s_{3}}}|\tau|^{j-\frac{1}{p}},
$$

where now $\tau^{2}=\gamma+2^{2 l} \xi^{\prime 2}+2^{2 l} \xi_{0} i$. Moreover,

$$
c_{1} 2^{l} \leq|\tau| \leq c_{2} 2^{l} \quad \text { for } \bar{\xi} \in A,
$$

if we assume that $\gamma<2^{2 l+r} \mid$ for $l \in \mathbb{N} \cup\{0\}$ and some $r \in \mathbb{N} \cup\{0\}$.

Finally, since $\left\{\phi_{k}\right\} \in \mathcal{A}_{a l}\left(\mathbb{R}^{3}\right)$ (see Section 2) we have

$$
\begin{array}{r}
\left\|\partial_{\xi^{\prime}}^{s_{4}^{\prime}} \partial_{\xi_{0}}^{s_{4}} \phi_{k}\left(2^{2 l} \cdot, 2^{l} \cdot\right)\right\|_{L_{2}(A)}=\| \partial_{\xi^{\prime}}^{s_{4}^{\prime}} \partial_{\xi_{0}}^{s_{4}} \phi_{k}\left(2^{2 k} \cdot 2^{2(l-k)} \cdot, \cdot 2^{k} \cdot 2^{(l-k)} \cdot \|_{L_{2}(A)}\right. \\
\leq c 2^{(d+2)|l-k|}\left\|\partial_{y^{\prime}}^{s_{4}^{\prime}} \partial_{y_{0}}^{s_{4}} \phi_{k}\left(2^{2 k} \cdot, 2^{k} \cdot\right)\right\|_{L_{2}(B)} \leq c 2^{(d+4-L)|l-k|}
\end{array}
$$

where $L$ is chosen sufficiently large,

$$
\begin{array}{ll}
B=\left\{\bar{y}: 2^{l-k-2} \leq|\bar{y}|_{a} \leq 2^{l-k+2}\right\} & \text { for } l \neq 0, \\
B=\left\{\bar{y}:|\bar{y}|_{a} \leq 2^{l-k+2}\right\} & \text { for } l=0
\end{array}
$$

and where we have used the change of variables $y_{0}=2^{2(l-k)} \xi_{0}, y^{\prime}=2^{(l-k)} \xi^{\prime}$.

In view of estimates (3.31)-(3.39), inequality (3.29) follows. 
Now, we shall derive the estimate for

$$
\|u\|_{2, B_{p, q, \gamma}^{\frac{\sigma}{2}, \sigma}\left(\mathbb{R}_{+} \times \mathbb{R}_{+}^{3}\right)} \equiv\left[\sum_{k=0}^{\infty}\left(\int_{\mathbb{R}} \int_{\mathbb{R} \times \mathbb{R}_{+}^{3}} \frac{\left|e^{-\gamma x_{0}}\left(F_{2}^{-1} \phi_{k} F_{2} U\right)\left(\bar{x}, x_{3}, z\right)\right|^{p}}{|z|^{1+p(\sigma-[\sigma])}} d \bar{x} d x_{3} d z\right)^{\frac{q}{p}}\right]^{\frac{1}{q}},
$$

where $U\left(\bar{x}, x_{3}, z\right)=\partial_{x_{3}}^{[\sigma]} u\left(\bar{x}, x_{3}+z\right)-\partial_{x_{3}}^{[\sigma]} u\left(\bar{x}, x_{3}\right)$.

LEMma 3.7. Let the assumptions of Theorem 3.4 be satisfied. Then

$$
\begin{aligned}
& \|u\|_{2, B_{p, q, \gamma}^{\frac{\sigma}{2}, \sigma}\left(\mathbb{R}_{+} \times \mathbb{R}_{+}^{3}\right)} \leq c\left(\sum_{k=1}^{2}\left\|b_{k}\right\|_{B_{p, q, \gamma}^{\left(\sigma-1-\frac{1}{p}\right) / 2,\left(\sigma-1-\frac{1}{p}\right)}\left(\mathbb{R}_{+} \times \mathbb{R}^{2}\right)}\right) . \\
& \left.\quad+\left\|b_{3}\right\|_{B_{p, q, \gamma}^{\left(\sigma-\frac{1}{p}\right) / 2, \sigma-\frac{1}{p}}\left(\mathbb{R}_{+} \times \mathbb{R}^{2}\right)}\right) .
\end{aligned}
$$

Proof. Since

$$
F_{2} U_{m}=\sum_{r=1}^{3}\left(g_{m r} \partial_{x_{3}}^{[\sigma]} E_{0}+h_{m r} \partial_{x_{3}}^{[\sigma]} E_{1}\right) \hat{b}_{r}
$$

where $E_{0}=e_{0}\left(\bar{\xi}, x_{3}+z\right)-e_{0}\left(\bar{\xi}, x_{3}\right), E_{1}=e_{1}\left(\bar{\xi}, x_{3}+z\right)-e_{1}\left(\bar{\xi}, x_{3}\right)$, we have

$$
\begin{aligned}
& \|u\|_{2, B_{p, q, \gamma}^{\frac{\sigma}{2}, \sigma}\left(\mathbb{R}_{+} \times \mathbb{R}_{+}^{3}\right)} \\
& =\left[\sum_{k=0}^{\infty}\left(\int_{\mathbb{R}} \int_{\mathbb{R} \times \mathbb{R}_{+}^{3}} \frac{\left|e^{-\gamma x_{0}} F_{2}^{-1} \phi_{k} \sum_{r=1}^{3}\left(g_{m r} \partial_{x_{3}}^{[\sigma]} E_{0}+h_{m r} \partial_{x_{3}}^{[\sigma]} E_{1}\right) \hat{b}_{r}\right|^{p}}{z^{1+p(\sigma-[\sigma])}}\right)^{\frac{q}{p}}\right]^{\frac{1}{q}} \\
& \leq c\left(\sum_{k=0}^{\infty} \sum_{r=1}^{3} J_{k r}^{q}\right)^{1 / q}
\end{aligned}
$$

where

$$
J_{2 k r} \equiv\left(\int_{\mathbb{R}} \int_{\mathbb{R} \times \mathbb{R}_{+}^{3}} \frac{\left|e^{-\gamma x_{0}} F_{2}^{-1} \phi_{k}\left(g_{m r} \partial_{x_{3}}^{[\sigma]} E_{0}+h_{m r} \partial_{x_{3}}^{[\sigma]} E_{1}\right) \hat{b}_{r}\right|^{p}}{z^{1+p(\sigma-[\sigma])}} d \bar{x} d x_{3} d z\right)^{1 / p} .
$$

Introducing the same family of functions $\left\{\psi_{l}(\bar{\xi})\right\}$ as in the proof of Lemma 3.5 we get

$$
\begin{aligned}
J_{2 k r}= & \left(\int_{\mathbb{R}} \int_{\mathbb{R} \times \mathbb{R}_{+}^{3}} \mid \sum_{l=0}^{\infty} \frac{e^{-\gamma x_{0}}\left[F_{2}^{-1} \psi_{l}\left(g_{m r} \partial_{x_{3}}^{[\sigma]} E_{0}+h_{m r} \partial_{x_{3}}^{[\sigma]} E_{1}\right)\right.}{|z|^{1 / p+(\sigma-[\sigma])}}\right. \\
& \cdot \frac{\left.F_{2} F_{2}^{-1} \phi_{k} F_{2} F_{2}^{-1} \phi_{l} F_{2} b_{r}\right]\left(\bar{x}, x_{3}, z\right)}{\left.\left.|z|^{1 / p+(\sigma-[\sigma])}\right|^{p} d \bar{x} d x_{3} d z\right)^{1 / p}} \\
= & \left(\int_{\mathbb{R}} \int_{\mathbb{R}^{3} \times \mathbb{R}_{+}} \mid \sum_{l=0}^{\infty} \frac{e^{-\gamma x_{0}}\left[F_{2}^{-1} \psi_{l}\left(g_{m r} \partial_{x_{3}}^{[\sigma]} E_{0}+h_{m r} \partial_{x_{3}}^{[\sigma]} E_{1}\right)\right.}{|z|^{1 / p+(\sigma-[\sigma])}}\right. \\
& \left.\left.\cdot \frac{\left.F_{2} F_{2}^{-1} \phi_{k} * F_{2} F_{2}^{-1} \phi_{l} F_{2} b_{r}\right]\left(\bar{x}, x_{3}, z\right)}{|z|^{1 / p+(\sigma-[\sigma])}}\right|^{p} d \bar{x} d x_{3} d z\right)^{1 / p} \\
= & \left(\int_{\mathbb{R}} \int_{\mathbb{R}^{3} \times \mathbb{R}_{+}} \mid \sum_{l=0}^{\infty} e^{-\gamma x_{0}} \int_{\mathbb{R}^{3}} d \bar{y} \frac{\left[F_{2}^{-1} \psi_{l}\left(g_{m r} \partial_{x_{3}}^{[\sigma]} E_{0}+h_{m r} \partial_{x_{3}}^{[\sigma]} E_{1}\right) F_{2} F_{2}^{-1} \phi_{k}\right]\left(\bar{y}, x_{3}\right)}{|z|^{1 / p+(\sigma-[\sigma])}}\right. \\
& \left.\left.\cdot \frac{\left(F_{2}^{-1} \phi_{l} F_{2} b_{r}\right)(\bar{x}-\bar{y})}{|z|^{1 / p+(\sigma-[\sigma])}}\right|^{p} d \bar{x} d x_{3} d z\right)^{1 / p} \cdot
\end{aligned}
$$


Now, using formula (3.26) from Lemma 3.5 with $\partial_{x_{3}}^{j} e_{i}(i=0,1)$ replaced by $\partial_{x_{3}}^{j} E_{i}$ $(i=0,1)$ we obtain

$$
\begin{aligned}
J_{2 k r}= & {\left[\int_{\mathbb{R}} \int_{\mathbb{R}^{3} \times \mathbb{R}_{+}} \mid \sum_{l=0}^{\infty} e^{-\gamma x_{0}} 2^{-4 l} \int_{\mathbb{R}^{3}} d \bar{w} \frac{\left[F_{2}^{-1} \psi_{l}\left(g_{m r} \partial_{x_{3}}^{[\sigma]} E_{0}+h_{m r} \partial_{x_{3}}^{[\sigma]} E_{1}\right) *\right.}{|z|^{1 / p+\sigma-[\sigma]}}\right.} \\
& \left.\left.\frac{\left.* F_{2}^{-1} \phi_{k}\right]\left(2^{-l} \tilde{w}, x_{3}, z\right) \cdot\left(F_{2}^{-1} \phi_{l} F_{2} b_{r}\right)\left(\bar{x}-2^{-l} \tilde{w}\right)}{|z|^{1 / p+\sigma-[\sigma]}}\right|^{p} d \bar{x} d x_{3} d z\right]^{1 / p},
\end{aligned}
$$

where we used the change of variables $y_{i}=2^{-l} w_{i}, i=1,2, y_{0}=2^{-2 l} w_{0}$ and the notation $\tilde{w}=\left(2^{-l} w_{0}, w^{\prime}\right), w^{\prime}=\left(w_{1}, w_{2}\right), \bar{w}=\left(w^{\prime}, w_{0}\right)$.

Next, formula (3.27) from Lemma 3.5 yields

$$
\begin{aligned}
& J_{2 k r}=\left[\int_{\mathbb{R}_{+}} d x_{3} \int_{\mathbb{R}^{3}} d \bar{x} \int_{\mathbb{R}} \frac{d z}{|z|^{1+p(\sigma-[\sigma])}} \mid \sum_{l=0}^{\infty} \int_{\mathbb{R}^{3}} d \bar{w} e^{-\gamma w_{0}}\right. \\
& \cdot\left\{\left[F_{2}^{-1} \psi_{l}\left(g_{m r} \partial_{x_{3}}^{[\sigma]} E_{0}+h_{m r} \partial_{x_{3}}^{[\sigma]} E_{1}\right)\right]\left(2^{2 l} \cdot, 2^{l} \cdot\right) *\left(F_{2}^{-1} \phi_{k}\right)\left(2^{2 l} \cdot, 2^{l} \cdot\right)\right\}\left(\bar{w}, x_{3}, z\right) \\
& \left.\left.\cdot e^{-\gamma\left(x_{0}-w_{0}\right)}\left(F_{2}^{-1} \phi_{k} F_{2} b_{r}\right)(\bar{x}-\bar{w})\right|^{p}\right]^{\frac{1}{p}} \\
& \leq\left\{\int_{\mathbb{R}_{+}} d x_{3} \int_{\mathbb{R}} \frac{d z}{|z|^{1+p(\sigma-[\sigma])}} \mid \sum_{l=0}^{\infty} \int_{\mathbb{R}^{3}} d \bar{w} e^{-\gamma w_{0}}\left\{\left[F _ { 2 } ^ { - 1 } \psi _ { l } \left(g_{m r} \partial_{x_{3}}^{[\sigma]} E_{0}\right.\right.\right.\right. \\
& \left.\left.\left.+h_{m r} \partial_{x_{3}}^{[\sigma]} E_{1}\right)\right]\left(2^{2 l} \cdot, 2^{l} \cdot\right) *\left(F_{2}^{-1} \phi_{k}\right)\left(2^{2 l} \cdot, 2^{l} \cdot\right)\left(\bar{w}, x_{3}, z\right)\right\}\left(\int_{\mathbb{R}^{3}} d \bar{x} \mid e^{-\gamma\left(x_{0}-w_{0}\right)}\right. \\
& \left.\left.\left.\cdot\left(F_{2}^{-1} \phi_{l} F_{2} b_{r}\right)(\bar{x}-\bar{w})\right|^{p}\right)\left.^{1 / p}\right|^{p}\right\}^{1 / p} \equiv J_{2 k r}^{1}
\end{aligned}
$$

where we also used the Minkowski inequality. Applying the change of variables $\bar{\zeta}=\bar{x}-\bar{w}$ in the integral with respect to $\bar{x}$ gives

$$
\begin{aligned}
J_{2 k r}^{1}= & \left\{\int_{\mathbb{R}_{+}} d x_{3} \int_{\mathbb{R}} \frac{d z}{|z|^{1+p(\sigma-[\sigma])}} \mid \sum_{l=0}^{\infty} \int_{\mathbb{R}^{3}} d \bar{w} e^{-\gamma w_{0}}\left\{\left[F _ { 2 } ^ { - 1 } \psi _ { l } \left(g_{m r} \partial_{x_{3}}^{[\sigma]} E_{0}\right.\right.\right.\right. \\
& \left.\left.\left.+h_{m r} \partial_{x_{3}}^{[\sigma]} E_{1}\right)\right]\left(2^{2 l} \cdot, 2^{l} \cdot\right) *\left(F_{2}^{-1} \phi_{k}\right)\left(2^{2 l} \cdot 2^{l} \cdot\right)\right\}\left(\bar{w}, x_{3}, z\right) \\
& \left.\left.\cdot\left(\int_{\mathbb{R}^{3}} d \bar{\zeta}\left|e^{-\gamma \zeta_{0}}\left(F_{2}^{-1} \phi_{l} F_{2} b_{r}\right)(\bar{\zeta})\right|^{p}\right)^{1 / p}\right|^{p}\right\}^{1 / p},
\end{aligned}
$$

where $\bar{\zeta}=\left(\zeta_{0}, \zeta^{\prime}\right)$. Using the Hölder inequality in the integral with respect to $\bar{w}$ and replacing $\bar{w}$ by $\bar{y}$ and $\bar{\zeta}$ by $\bar{x}$ we get

$$
\begin{aligned}
J_{2 k r}^{1} \leq & \left\{\int_{\mathbb{R}_{+}} d x_{3} \int_{\mathbb{R}} \frac{d z}{|z|^{1+p(\sigma-[\sigma])}} \mid \sum_{l=0}^{\infty}\left(\int_{\mathbb{R}^{3}} d \bar{y} \frac{1}{\left(1+|\bar{y}|_{a}\right)^{d}}\right)^{1 / 2}\right. \\
& \cdot\left(\int_{\mathbb{R}^{3}} d \bar{y} \mid e^{-\gamma y_{0}}\left\{\left[F_{2}^{-1} \psi_{l}\left(g_{m r} \partial_{x_{3}}^{[\sigma]} E_{0}+h_{m r} \partial_{x_{3}}^{[\sigma]} E_{1}\right)\right]\left(2^{2 l} \cdot, 2^{l} \cdot\right)\right.\right. \\
& \left.\left.\left.*\left(F_{2}^{-1} \phi_{k}\right)\left(2^{2 l} \cdot, 2^{l} \cdot\right)\right\}\left.\left(\bar{y}, x_{3}, z\right)\left(1+|\bar{y}|_{a}\right)^{\frac{d}{2}}\right|^{2}\right)\left.^{1 / 2}\right|^{p}\right\}^{1 / p} \\
& \cdot\left\|e^{-\gamma x_{0}} F_{1}^{-1} \phi_{l} F_{1} b_{r}\right\|_{L_{p}\left(\mathbb{R}^{3}\right)} .
\end{aligned}
$$


Hence, assuming that $d>3$ and applying the Parseval identity we obtain

$$
\begin{aligned}
J_{2 k r}^{2} \leq & c\left(\int_{\mathbb{R}_{+}} d x_{3} \int_{\mathbb{R}} \frac{d z}{|z|^{1+p(\sigma-[\sigma])}} \mid \sum_{l=0}^{\infty} \|\left[\psi _ { l } \left(g_{m r} \partial_{x_{3}}^{[\sigma]} E_{0}\right.\right.\right. \\
& \left.\left.\left.+h_{m r} \partial_{x_{3}}^{[\sigma]} E_{1}\right)\right]\left(2^{2 l} \cdot, 2^{l} \cdot, x_{3}, z\right) \phi_{k}\left(2^{2 l} \cdot, 2^{l} \cdot\right) \|\left._{W_{2}^{\frac{d}{2}, d}\left(\mathbb{R}^{3}\right)}\right|^{p}\right)^{1 / p} \\
& \cdot\left\|e^{-\gamma x_{0}} F_{1}^{-1} \phi_{l} F_{1} b_{r}\right\|_{L_{p}\left(\mathbb{R}^{3}\right)} .
\end{aligned}
$$

Lemma 3.8 below implies

$$
J_{2 k r}^{2} \leq c \sum_{l=0}^{\infty} 2^{l\left(\sigma-\frac{1}{p}-c_{r}\right)} 2^{\left(d+4-L_{1}\right)|l-k|}\left\|e^{-\gamma x_{0}} F_{1}^{-1} \psi_{l} F_{1} b_{r}\right\|_{L_{p}\left(\mathbb{R}^{3}\right)},
$$

where $c_{r}=1$ for $r=1,2, c_{r}=0$ for $r=3$. Hence

$$
\begin{aligned}
& \left\|u_{m}\right\|_{2, B_{p, q, \gamma}}^{\frac{\sigma}{2}, \sigma}\left(\mathbb{R}_{+} \times \mathbb{R}_{+}^{3}\right) \\
& \quad \leq c\left(\sum_{k=0}^{\infty} \sum_{r=1}^{3} \sum_{l=0}^{\infty} 2^{l\left(\sigma-\frac{1}{p}-c_{r}\right) q} 2^{\left(\varepsilon+d+4-L_{1}\right)|l-k| q}\left\|e^{-\gamma x_{0}} F_{1}^{-1} \phi_{l} F_{1} b_{r}\right\|_{L_{p}\left(\mathbb{R}^{3}\right)}^{q}\right)^{1 / q},
\end{aligned}
$$

where $\varepsilon>0$ is arbitrarily small. Let $L_{1}>d+4+\varepsilon$. Then (3.40) follows.

Now, we prove

Lemma 3.8. Let $d>3$. Then

$$
\begin{aligned}
K_{m r} \equiv & \left(\int_{\mathbb{R}_{+}} d x_{3} \int_{\mathbb{R}} \frac{d z}{|z|^{1+p(\sigma-[\sigma])}} \|\left[\psi _ { l } \left(g_{m r} \partial_{x_{3}}^{[\sigma]} E_{0}\right.\right.\right. \\
& \left.\left.\left.+h_{m r} \partial_{x_{3}}^{[\sigma]} E_{1}\right)\right]\left(2^{2 l} \cdot, 2^{l} \cdot, x_{3}, z\right) \phi_{k}\left(2^{2 l} \cdot, 2^{l} \cdot\right) \|_{W_{2}^{\frac{d}{2}, d}}^{p}{ }_{\left(\mathbb{R} \times \mathbb{R}^{2}\right)}\right)^{1 / p} \\
\leq & c 2^{l\left(\sigma-\frac{1}{p}-c_{r}\right)} 2^{\left(d+4-L_{1}\right)|l-k|},
\end{aligned}
$$

where for $l=0$ the constant $c$ depends on $\gamma, L_{1}$ can be chosen sufficiently large.

Proof. Using the Minkowski inequality as in Lemma 3.6 we obtain

$$
\begin{aligned}
K_{m r} \leq & c\left(\int_{\mathbb{R}_{+}} d x_{3} \int_{\mathbb{R}} \frac{d z}{|z|^{1+p(\sigma-[\sigma])}} \sum_{\sum_{i=1}^{4}\left(s_{i}^{\prime}+2 s_{i}\right) \leq d} \|\left(\partial_{\xi^{\prime}}^{s_{1}^{\prime}} \partial_{\xi_{0}}^{s_{1}} \psi_{l}\right)\left(2^{2 l} \cdot, 2^{l} \cdot\right)\right. \\
& \cdot\left(\partial_{\xi^{\prime}}^{s_{2}^{\prime}} \partial_{\xi_{0}}^{s_{2}} g_{m r} \partial_{\xi^{\prime}}^{s_{3}^{\prime}} \partial_{\xi_{0}}^{s_{3}} \partial_{x_{3}}^{[\sigma]} E_{0}+\partial_{\xi^{\prime}}^{s_{2}^{\prime}} \partial_{\xi_{0}}^{s_{2}} h_{m r} \partial_{\xi^{\prime}}^{s_{3}^{\prime}} \partial_{\xi_{0}}^{s_{3}} \partial_{x_{3}}^{[\sigma]} E_{1}\right)\left(2^{2 l} \cdot, 2^{l} \cdot, x_{3}\right) \\
& \left.\cdot\left(\partial_{\xi^{\prime}}^{s_{4}^{\prime}} \partial_{\xi_{0}}^{s_{4}} \phi_{k}\right)\left(2^{2 l} \cdot, 2^{l} \cdot\right) \|_{L_{2}\left(\mathbb{R}^{3}\right)}^{p}\right)^{1 / p} \\
\leq & c \sum \sum_{\sum_{i=1}^{4}\left(s_{i}^{\prime}+2 s_{i}\right) \leq d} \|\left(\partial_{\xi^{\prime}}^{s_{1}^{\prime}} \partial_{\xi_{0}}^{s_{1}} \psi_{l}\right)\left(2^{2 l} \cdot, 2^{l} \cdot\right) \partial_{\xi^{\prime}}^{s_{2}^{\prime}} \partial_{\xi_{0}}^{s_{2}} g_{m r}\left(2^{2 l} \cdot, 2^{l} \cdot\right) \\
& \cdot\left\|\frac{\partial_{\xi^{\prime}}^{s_{3}^{\prime}} \partial_{\xi_{0}}^{s_{3}} \partial_{x_{3}}^{[\sigma]} E_{0}}{|z|^{1 / p+(\sigma-[\sigma])}}\right\|_{L_{p}\left(\mathbb{R}_{+} \times \mathbb{R}\right)}\left(2^{2 l} \cdot, 2^{l} \cdot\right)\left(\partial_{\xi^{\prime}}^{s_{4}^{\prime}} \partial_{\xi_{0}}^{s_{4}} \phi_{k}\right)\left(2^{2 l} \cdot, 2^{l} \cdot\right) \|_{L_{2}\left(\mathbb{R}^{3}\right)}
\end{aligned}
$$




$$
\begin{aligned}
& +c \sum_{\sum_{i=1}^{4}\left(s_{i}^{\prime}+2 s_{i}\right) \leq d} \|\left(\partial_{\xi^{\prime}}^{s_{1}^{\prime}} \partial_{\xi_{0}}^{s_{1}} \psi_{l}\right)\left(2^{2 l} \cdot, 2^{l} \cdot\right) \partial_{\xi^{\prime}}^{s_{2}^{\prime}} \partial_{\xi_{0}}^{s_{2}} h_{m r}\left(2^{2 l} \cdot, 2^{l} \cdot\right) \\
& \cdot\left\|\frac{\partial_{\xi^{\prime}}^{s_{3}^{\prime}} \partial_{\xi_{0}}^{s_{3}} \partial_{x_{3}}^{[\sigma]} E_{1}}{|z|^{1 / p+(\sigma-[\sigma])}}\right\|_{L_{p}\left(\mathbb{R}_{+} \times \mathbb{R}\right)}\left(2^{2 l} \cdot, 2^{l} \cdot\right)\left(\partial_{\xi^{\prime}}^{s_{4}^{\prime}} \partial_{\xi_{0}}^{s_{4}} \phi_{k}\right)\left(2^{2 l} \cdot, 2^{l} \cdot\right) \|_{L_{2}\left(\mathbb{R}^{3}\right)} .
\end{aligned}
$$

Lemma 3.3 implies

$$
\left\|\frac{\partial_{\xi^{\prime}}^{s_{2}^{\prime}} \partial_{\xi_{0}}^{s_{2}} \partial_{x_{3}}^{[\sigma]} E_{0}}{|z|^{1 / p+(\sigma-[\sigma])}}\right\|_{L_{p}\left(\mathbb{R}_{+} \times \mathbb{R}\right)}\left(2^{2 l} \cdot, 2^{l} \cdot\right) \leq c 2^{l\left(s_{2}^{\prime}+2 s_{2}\right)}|\tau|^{\sigma-s_{2}^{\prime}-2 s_{2}-\frac{1}{p}-1}
$$

and

$$
\left\|\frac{\partial_{\xi^{\prime}}^{s_{2}^{\prime}} \partial_{\xi_{0}}^{s_{2}} \partial_{x_{3}}^{[\sigma]} E_{1}}{|z|^{1 / p+(\sigma-[\sigma])}}\right\|_{L_{p}\left(\mathbb{R}_{+} \times \mathbb{R}\right)}\left(2^{2 l} \cdot, 2^{l} \cdot\right) \leq c 2^{l\left(s_{2}^{\prime}+2 s_{2}\right)}|\tau|^{\sigma-s_{2}^{\prime}-2 s_{2}-\frac{1}{p}}
$$

Hence

$$
\begin{aligned}
& \left\|\frac{\partial_{\xi^{\prime}}^{s_{2}^{\prime}} \partial_{\xi_{0}}^{s_{2}} \partial_{x_{3}}^{[\sigma]} E_{0}}{|z|^{1 / p+(\sigma-[\sigma])}}\right\|_{L_{p}\left(\mathbb{R}_{+} \times \mathbb{R}\right)}\left(2^{2 l} \cdot, 2^{l} \cdot\right) \leq c 2^{l\left(\sigma-\frac{1}{p}-1\right)} \quad \text { for } \bar{\zeta} \in A \text { if } l \neq 0, \\
& \left\|\frac{\partial_{\xi^{\prime}}^{s_{2}^{\prime}} \partial_{\xi_{0}}^{s_{2}} \partial_{x_{3}}^{[\sigma]} E_{1}}{|z|^{1 / p+(\sigma-[\sigma])}}\right\|_{L_{p}\left(\mathbb{R}_{+} \times \mathbb{R}\right)}\left(2^{2 l} \cdot, 2^{l} \cdot\right) \leq c 2^{l\left(\sigma-\frac{1}{p}\right)} \quad \text { for } \bar{\xi} \in A \text { if } l \neq 0, \\
& \sum_{i=0}^{1}\left\|\frac{\partial_{\xi^{\prime}}^{s_{2}^{\prime}} \partial_{\xi_{0}}^{s_{2}} \partial_{x_{3}}^{[\sigma]} E_{i}}{|z|^{1 / p+(\sigma-[\sigma])}}\right\|_{L_{p}\left(\mathbb{R}_{+} \times \mathbb{R}\right)}\left(2^{2 l} \cdot, 2^{l} \cdot\right) \leq c(\gamma) \quad \text { for } \bar{\zeta} \in A \text { if } l=0,
\end{aligned}
$$

where $A$ is defined in Lemma 3.6. Now, by the above estimates and estimates (3.30)-(3.39), inequality (3.41) follows.

4. The parabolic problem with $f \neq 0, b_{k} \neq 0, u_{0}=0$ in the half-space. In this section we consider the problem

$$
\begin{array}{ll}
u_{t}-\operatorname{div} \mathbb{D}(u)=f & \text { in } \mathbb{R}_{+} \times \mathbb{R}_{+}^{3}, \\
\mu\left(\frac{\partial u_{k}}{\partial x_{3}}+\frac{\partial u_{3}}{\partial x_{k}}\right)=b_{k}, \quad k=1,2, & \text { in } \mathbb{R}_{+} \times \mathbb{R}^{2}, \\
u_{3}=b_{3} & \text { in } \mathbb{R}_{+} \times \mathbb{R}^{2}, \\
\left.u\right|_{t=0}=0 & \text { in } \mathbb{R}_{+}^{3} .
\end{array}
$$

LEMMA 4.1. Let $f \in B_{p, q, \gamma}^{\frac{\sigma}{2}-1, \sigma-2}\left(\mathbb{R}_{+} \times \mathbb{R}_{+}^{3}\right), b_{k} \in B_{p, q, \gamma}^{\left(\sigma-1-\frac{1}{p}\right) / 2, \sigma-1-\frac{1}{p}}\left(\mathbb{R}_{+} \times \mathbb{R}^{2}\right), k=1,2$, $b_{3} \in B_{p, q, \gamma}^{\left(\sigma-\frac{1}{p}\right) / 2, \sigma-\frac{1}{p}}\left(\mathbb{R}_{+} \times \mathbb{R}^{2}\right), 2<\sigma \in \mathbb{R}, p, q \in(1, \infty)$. Then there exists a unique solution to problem (4.1) such that $u \in B_{p, q, \gamma}^{\frac{\sigma}{2}, \sigma}\left(\mathbb{R}_{+} \times \mathbb{R}_{+}^{3}\right)$ and

$$
\begin{aligned}
& \|u\|_{B_{p, q, \gamma}^{\frac{\sigma}{2}, \sigma}\left(\mathbb{R}_{+} \times \mathbb{R}_{+}^{3}\right)} \leq c\left(\|f\|_{B_{p, q, \gamma}^{\frac{\sigma}{2}-1, \sigma-2}\left(\mathbb{R}_{+} \times \mathbb{R}_{+}^{3}\right)}\right. \\
& \left.\quad+\sum_{k=1}^{2}\left\|b_{k}\right\|_{B_{p, q, \gamma}^{\left(\sigma-1-\frac{1}{p}\right) / 2, \sigma-1-\frac{1}{p}}\left(\mathbb{R}_{+} \times \mathbb{R}^{2}\right)}+\left\|b_{3}\right\|_{B_{p, q, \gamma}^{\left(\sigma-\frac{1}{p}\right) / 2, \sigma-\frac{1}{p}}} \mathbb{R}_{+} \times \mathbb{R}^{2}\right) \\
& \quad),
\end{aligned}
$$

where $c>0$ is a constant independent of $u$. 
Proof. Since $f \in B_{p, q, \gamma}^{\frac{\sigma}{2}-1, \sigma-2}\left(\mathbb{R}_{+} \times \mathbb{R}_{+}^{3}\right)$, we can extend $f$ by zero to the function $f^{\prime}$ defined in $\mathbb{R} \times \mathbb{R}_{+}^{3}$, and then by Lemma 2.2 we can extend $f_{\gamma}=e^{-\gamma t} f^{\prime}$ onto $\mathbb{R} \times \mathbb{R}^{3}$ to a function $\tilde{f}_{\gamma}$ satisfying

$$
\left\|\tilde{f}_{\gamma}\right\|_{B_{p, q}^{\frac{\sigma}{2}-1, \sigma-2}\left(\mathbb{R} \times \mathbb{R}^{3}\right)} \leq c\left\|f_{\gamma}\right\|_{B_{p, q}^{\frac{\sigma}{2}-1, \sigma-2}\left(\mathbb{R} \times \mathbb{R}_{+}^{3}\right)}=\|f\|_{B_{p, q, \gamma}^{\frac{\sigma}{2}-1, \sigma-2}\left(\mathbb{R}_{+} \times \mathbb{R}_{+}^{3}\right)} .
$$

Let $\tilde{f}=\tilde{f}_{\gamma} e^{\gamma t}$. Then (4.3) yields

$$
\|\tilde{f}\|_{B_{p, q, \gamma}^{\frac{\sigma}{2}-1, \sigma-2}\left(\mathbb{R}_{+} \times \mathbb{R}^{3}\right)} \leq c\|f\|_{B_{p, q, \gamma}^{\frac{\sigma}{2}-1, \sigma-2}\left(\mathbb{R}_{+} \times \mathbb{R}_{+}^{3}\right)} .
$$

Consider the Cauchy problem

$$
\begin{array}{lll}
\tilde{u}_{t}-\operatorname{div} \mathbb{D}(\tilde{u})=\tilde{f} & \text { in } \mathbb{R}_{+} \times \mathbb{R}^{3}, \\
\left.\tilde{u}\right|_{t=0}=0 & \text { in } \mathbb{R}^{3} .
\end{array}
$$

By Lemma 4.3 below and (4.4)

$$
\|\tilde{u}\|_{B_{p, q, \gamma}^{\frac{\sigma}{2}, \sigma}\left(\mathbb{R}_{+} \times \mathbb{R}^{3}\right)} \leq c\|f\|_{B_{p, q, \gamma}^{\frac{\sigma}{2}-1, \sigma-2}\left(\mathbb{R}_{+} \times \mathbb{R}_{+}^{3}\right)} .
$$

Let $v=u-\tilde{u}$. Then $v$ is a solution of the problem

$$
\begin{array}{ll}
v_{t}-\operatorname{div} \mathbb{D}(v)=0 & \text { in } \mathbb{R} \times \mathbb{R}_{+}^{3}, \\
\left.\mu\left(\frac{\partial v_{2}}{\partial x_{3}}+\frac{\partial v_{3}}{\partial x_{k}}\right)\right|_{x_{3}=0}=b_{k}-\left.\mu\left(\frac{\partial \tilde{u}_{k}}{\partial x_{3}}+\frac{\partial \tilde{u}_{3}}{\partial x_{k}}\right)\right|_{x_{3}=0}, k=1,2, & \text { on } \mathbb{R} \times \mathbb{R}^{2}, \\
\left.v_{3}\right|_{x_{3}=0}=b_{3}-\left.\tilde{u}_{3}\right|_{x_{3}=0} & \text { on } \mathbb{R} \times \mathbb{R}^{2}, \\
\left.v\right|_{t=0}=0 & \text { in } \mathbb{R}_{+}^{3} .
\end{array}
$$

Let

$$
\tilde{u}_{\gamma}= \begin{cases}\tilde{u} e^{-\gamma t} & \text { for } t>0 \\ 0 & \text { for } t<0 .\end{cases}
$$

By the traces theorems (see [6]), estimate (4.5) and the fact that

$$
\left(\left.\mu\left(\frac{\partial \tilde{u}_{k}}{\partial x_{3}}+\frac{\partial \tilde{u}_{3}}{\partial x_{k}}\right)\right|_{x_{3}=0}\right)_{\gamma}=\left.\mu\left(\frac{\partial \tilde{u}_{k \gamma}}{\partial x_{3}}+\frac{\partial \tilde{u}_{3 \gamma}}{\partial x_{k}}\right)\right|_{x_{3}=0}
$$

and $\left(\left.\tilde{u}_{3}\right|_{x_{3}=0}\right)_{\gamma}=\left.\tilde{u}_{\gamma 3}\right|_{x_{3}=0}$ we have

$$
\begin{aligned}
& \left\|\left.\tilde{u}_{3}\right|_{x_{3}=0}\right\|_{B_{p, q, \gamma}^{\frac{\sigma}{2}-\frac{1}{2 p}, \sigma-\frac{1}{p}}\left(\mathbb{R}_{+} \times \mathbb{R}^{2}\right)}=\left\|\left.\tilde{u}_{3 \gamma}\right|_{x_{3}=0}\right\|_{B_{p, q}^{\frac{\sigma}{2}-\frac{1}{2 p}, \sigma-\frac{1}{p}}\left(\mathbb{R} \times \mathbb{R}^{2}\right)} \\
& \leq c\left\|\tilde{u}_{\gamma}\right\|_{B_{p, q}^{\frac{\sigma}{2}, \sigma}\left(\mathbb{R} \times \mathbb{R}^{3}\right)} \leq c\|f\|_{B_{p, q, \gamma}^{\frac{\sigma}{2}-1, \sigma-2}\left(\mathbb{R}_{+} \times \mathbb{R}_{+}^{3}\right)}
\end{aligned}
$$

and

$$
\begin{aligned}
& \left\|\left.\mu\left(\frac{\partial \tilde{u}_{k}}{\partial x_{3}}+\frac{\partial \tilde{u}_{3}}{\partial x_{k}}\right)\right|_{x_{3}=0}\right\|_{B_{p, q, \gamma}^{\frac{\sigma}{2}-\frac{1}{2}-\frac{1}{2 p}, \sigma-1-\frac{1}{p}}\left(\mathbb{R}_{+} \times \mathbb{R}^{2}\right)} \\
& =\left\|\left.\mu\left(\frac{\partial \tilde{u}_{k \gamma}}{\partial x_{3}}+\frac{\partial \tilde{u}_{3 \gamma}}{\partial x_{k}}\right)\right|_{x_{3}=0}\right\|_{B_{p, q}^{\frac{\sigma}{2}-\frac{1}{2}-\frac{1}{2 p}, \sigma-1-\frac{1}{p}}\left(\mathbb{R} \times \mathbb{R}^{2}\right)} \leq c\|f\|_{B_{p, q, \gamma}^{\frac{\sigma}{2}-1, \sigma-2}\left(\mathbb{R}_{+} \times \mathbb{R}_{+}^{3}\right)} .
\end{aligned}
$$

Theorem 3.4 and estimates (4.6)-(4.7) imply the existence of a unique solution to problem (4.1) and estimate (4.2).

This ends the proof. 
From Lemma 4.1 the corollary follows.

Corollary 4.2. Let $f \in B_{p, q}^{\frac{\sigma}{2}-1, \sigma-2}\left((0, T) \times \mathbb{R}_{+}^{3}\right), b_{k} \in B_{p, q}^{\left(\sigma-1-\frac{1}{p}\right) / 2, \sigma-1-\frac{1}{p}}\left((0, T) \times \mathbb{R}^{2}\right)$, $k=1,2, b_{3} \in B_{p, q}^{\left(\sigma-\frac{1}{p}\right) / 2, \sigma-\frac{1}{p}}\left((0, T) \times \mathbb{R}^{2}\right), 2<\sigma \in \mathbb{R}, p, q \in(1, \infty), 0<T<\infty$. Assume also that

$$
\begin{aligned}
& f^{\prime} \in B_{p, q}^{\frac{\sigma}{2}-1, \sigma-2}\left((-\infty, T) \times \mathbb{R}_{+}^{3}\right), \\
& b_{k}^{\prime} \in B_{p, q}^{\left(\sigma-1-\frac{1}{p}\right) / 2, \sigma-1-\frac{1}{p}}\left((-\infty, T) \times \mathbb{R}^{2}\right), \quad k=1,2, \\
& b_{3}^{\prime} \in B_{p, q}^{\left(\sigma-\frac{1}{p}\right) / 2, \sigma-\frac{1}{p}}\left((-\infty, T) \times \mathbb{R}^{2}\right),
\end{aligned}
$$

where

$$
h^{\prime}=\left\{\begin{array}{ll}
h & \text { for } t \in(0, T), \\
0 & \text { for } t<0,
\end{array} \quad h \in\left\{f, b_{1}, b_{2}, b_{3}\right\} .\right.
$$

Then there exists a unique solution $u \in B_{p, q}^{\frac{\sigma}{2}, \sigma}\left((0, T) \times \mathbb{R}_{+}^{3}\right)$ to the problem

$$
\begin{array}{ll}
u_{t}-\operatorname{div} \mathbb{D}(u)=f & \text { in }(0, T) \times \mathbb{R}_{+}^{3}, \\
\mu\left(\frac{\partial u_{k}}{\partial x_{3}}+\frac{\partial u_{3}}{\partial x_{k}}\right)=b_{k}, \quad k=1,2 & \text { on }(0, T) \times \mathbb{R}^{2}, \\
u_{3}=b_{3} & \text { on }(0, T) \times \mathbb{R}^{2}, \\
\left.u\right|_{t=0}=0 & \text { in } \mathbb{R}_{+}^{3} .
\end{array}
$$

Moreover, $u^{\prime} \in B_{p, q}^{\frac{\sigma}{2}, \sigma}\left((-\infty, T) \times \mathbb{R}_{+}^{3}\right)$ and we have the estimate

$$
\begin{aligned}
& \left\|u^{\prime}\right\|_{B_{p, q}^{\frac{\sigma}{2}, \sigma}\left((-\infty, T) \times \mathbb{R}_{+}^{3}\right)} \leq c(T)\left(\left\|f^{\prime}\right\|_{B_{p, q}^{\frac{\sigma}{2}-1, \sigma-2}\left((-\infty, T) \times \mathbb{R}_{+}^{3}\right)}\right. \\
& \quad+\sum_{k=1}^{2}\left\|b_{k}^{\prime}\right\|_{B_{p, q}^{\left(\sigma-1-\frac{1}{p}\right) / 2, \sigma-1-\frac{1}{p}}\left((-\infty, T) \times \mathbb{R}^{2}\right)}+\left\|b_{3}^{\prime}\right\|_{B_{p, q}^{\left(\sigma-\frac{1}{p}\right) / 2, \sigma-\frac{1}{p}}}\left((-\infty, T) \times \mathbb{R}^{2}\right) \\
& \quad),
\end{aligned}
$$

where $c=c(T)$ is an increasing function of $T$.

Proof. Using the extension theorem, we extend the functions $f_{\gamma}^{\prime}=e^{-\gamma t} f^{\prime}, b_{k \gamma}^{\prime}=e^{-\gamma t} b_{k}^{\prime}$ $(k=1,2,3)$ to functions $\tilde{f}_{\gamma}$ and $\tilde{b}_{k}$ such that

$$
\begin{gathered}
\left\|\hat{f}_{\gamma}\right\|_{B_{p, q}^{\frac{\sigma}{2}-1, \sigma-2}\left(\mathbb{R} \times \mathbb{R}_{+}^{3}\right)} \leq c\left\|f_{\gamma}^{\prime}\right\|_{B_{p, q}^{\frac{\sigma}{2}-1, \sigma-2}\left((-\infty, T) \times \mathbb{R}^{3}\right)} \\
\leq c\left\|f^{\prime}\right\|_{B_{p, q}^{\frac{\sigma}{2}-1, \sigma-2}\left((-\infty, T) \times \mathbb{R}_{+}^{3}\right)}, \\
\left\|\tilde{b}_{k \gamma}\right\|_{B_{p, q}^{\left(\sigma-1-\frac{1}{p}\right) / 2, \sigma-1-\frac{1}{p}}\left(\mathbb{R} \times \mathbb{R}_{+}^{3}\right)} \leq c\left\|b_{k \gamma}^{\prime}\right\|_{B_{p, q}^{\left(\sigma-1-\frac{1}{p}\right) / 2, \sigma-1-\frac{1}{p}}\left((-\infty, T) \times \mathbb{R}_{+}^{3}\right)} \\
\leq c\left\|b_{k}^{\prime}\right\|_{B_{p, q}^{\left(\sigma-1-\frac{1}{p}\right) / 2, \sigma-1-\frac{1}{p}}\left((-\infty, T) \times \mathbb{R}_{+}^{3}\right)}, \quad k=1,2, \\
\left\|\tilde{b}_{3 \gamma}\right\|_{B_{p, q}^{\left(\sigma-\frac{1}{p}\right) / 2, \sigma-\frac{1}{p}}\left(\mathbb{R} \times \mathbb{R}_{+}^{3}\right)} \leq c\left\|b_{3 \gamma}^{\prime}\right\|_{B_{p, q}^{\left(\sigma-\frac{1}{p}\right) / 2, \sigma-\frac{1}{p}}\left((-\infty, T) \times \mathbb{R}_{+}^{3}\right)}, \\
\leq c\left\|b_{3}^{\prime}\right\|_{B_{p, q}^{\left(\sigma-\frac{1}{p}\right) / 2, \sigma-\frac{1}{p}}\left((-\infty, T) \times \mathbb{R}_{+}^{3}\right)} .
\end{gathered}
$$




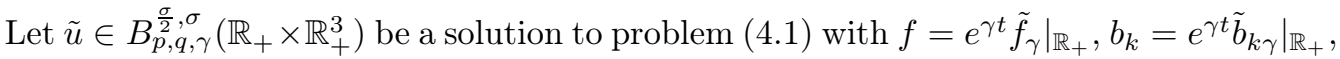
$k=1,2,3$. Then the function $u=\left.\tilde{u}\right|_{(0, T)} \in B_{p, q}^{\frac{\sigma}{2}, \sigma}\left((0, T) \times \mathbb{R}_{+}^{3}\right)$ satisfies problem (4.8). Moreover, $u^{\prime} \in B_{p, q}^{\frac{\sigma}{2}, \sigma}\left((-\infty, T) \times \mathbb{R}_{+}^{3}\right)$ and $u^{\prime}$ fulfills estimate (4.9).

Now, we consider the Cauchy problem

$$
\begin{array}{ll}
u_{t}-\operatorname{div} \mathbb{D}(u)=f & \text { in } \mathbb{R}_{+} \times \mathbb{R}^{3}, \\
\left.u\right|_{t=0}=0 & \text { in } \mathbb{R}^{3} .
\end{array}
$$

We have

LEMmA 4.3. Let $f \in B_{p, q, \gamma}^{\frac{\sigma}{2}-1, \sigma-2}\left(\mathbb{R}_{+} \times \mathbb{R}^{3}\right), 2<\sigma \in \mathbb{R}, p, q \in(1, \infty)$. Then there exists a unique solution of problem (4.10) such that $u \in B_{p, q, \gamma}^{\frac{\sigma}{2}, \sigma}\left(\mathbb{R}_{+} \times \mathbb{R}^{3}\right)$ and

$$
\|u\|_{B_{p, q, \gamma}^{\frac{\sigma}{2}, \sigma}\left(\mathbb{R}_{+} \times \mathbb{R}^{3}\right)} \leq c\|f\|_{B_{p, q, \gamma}^{\frac{\sigma}{2}-1, \sigma-2}\left(\mathbb{R}_{+} \times \mathbb{R}^{3}\right)} .
$$

Proof. We use the same argument as in Theorem 3.1. The Fourier-Laplace transform applied to (4.10) yields

$$
\begin{aligned}
& \left(s+\mu \xi^{2}\right) F_{1} u_{k}+\nu \xi_{k} \xi_{3} F_{1} u_{3}+\nu \xi_{k} \xi_{j} F_{1} u_{j}=F_{1} f_{k}, \quad k=1,2 \\
& {\left[s+\mu \xi^{\prime 2}+(\mu+\nu) \xi_{3}^{2}\right] F_{1} u_{3}+\nu \xi_{j} \xi_{3} F_{1} u_{j}=F_{1} f_{3},}
\end{aligned}
$$

where $\left(F_{1} u\right)(s, \xi)=\int_{\mathbb{R}_{+}} e^{-s t} d t \int_{\mathbb{R}^{3}} e^{-i x \cdot \xi} u(t, x) d x, s=\gamma+i \xi_{0}, \xi=\left(\xi_{1}, \xi_{2}, \xi_{3}\right)$. Solving (4.11) we get

$$
F_{1} u_{m}=\sum_{r=1}^{3} \frac{1}{s+\mu \xi^{2}} a_{m r} F_{1} f_{r}, \quad m=1,2,3
$$

where

$$
\begin{array}{lll}
a_{11}=\frac{s+\mu \xi^{2}+\nu \xi_{2}^{2}+2 \nu \xi_{3}^{2}}{A}, & a_{12}=\frac{-\nu \xi_{1} \xi_{2}}{A}, & a_{13}=-\frac{2 \nu \xi_{1} \xi_{3}}{A}, \\
a_{21}=\frac{-\nu \xi_{1} \xi_{2}}{A}, & a_{22}=\frac{s+\mu \xi^{2}+\nu \xi_{1}^{2}+2 \nu \xi_{3}^{2}}{A}, & a_{23}=\frac{-2 \nu \xi_{2} \xi_{3}}{A}, \\
a_{31}=\frac{-\nu \xi_{1} \xi_{3}}{A}, & a_{32}=\frac{-\nu \xi_{2} \xi_{3}}{A}, & a_{33}=\frac{s+\nu \xi^{2}+\nu \xi^{\prime 2}}{A}, \\
A=s+\mu \xi^{2}+\nu \xi^{2}+\nu \xi_{3}^{2} . & &
\end{array}
$$

As in Lemma 3.5 we consider

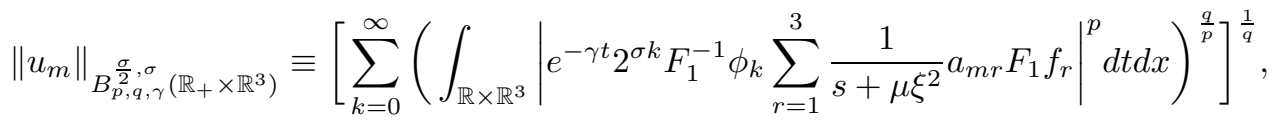

where $\left\{\phi_{k}\right\}_{k=1}^{\infty} \subset \Phi_{a}\left(\mathbb{R}^{4}\right)$ (see Definitions 2.2 and 2.1 with $\bar{x}, \bar{\alpha},|\bar{x}|_{a}$ replaced by

$$
\bar{x}=\left(x_{0}, x_{1}, x_{2}, x_{3}\right), \quad \bar{\alpha}=\left(\alpha_{0}, \alpha_{1}, \alpha_{2}, \alpha_{3}\right), \quad|\bar{x}|_{a}=\left(\left|x_{0}\right|+\sum_{i=1}^{3}\left|x_{i}\right|^{2}\right)^{1 / 2} .
$$

We also introduce a family of functions $\left\{\psi_{j}\left(\xi_{0}, \xi\right)\right\}$ with the properties: $\sum_{j=1}^{\infty} \psi_{j}\left(\xi_{0}, \xi\right)$ $=1, \operatorname{supp} \psi_{0} \subset\left\{\left(\xi_{0}, \xi\right):\left|\left(\xi_{0}, \xi\right)\right|_{a} \leq 4\right\}, \operatorname{supp} \psi_{j} \subset\left\{\left(\xi_{0}, \xi\right): 2^{j-2} \leq\left|\left(\xi_{0}, \xi\right)\right|_{a} \leq 2^{j+1}\right\}$, 
$\psi_{j}\left(\xi_{0}, \xi\right)=1$ for $\left(\xi_{0}, \xi\right) \in \operatorname{supp} \phi_{j},\left|\left(\xi_{0}, \xi\right)\right|_{a}=\left(\left|\xi_{0}\right|+\sum_{i=1}^{3}\left|\xi_{i}\right|^{2}\right)^{1 / 2}$. Then

$$
\left\|u_{m}\right\|_{B_{p, q, \gamma}^{\frac{\sigma}{2}, \sigma}\left(\mathbb{R}_{+} \times \mathbb{R}^{3}\right)} \leq c\left(\sum_{k=0}^{\infty} \sum_{r=1}^{3} I_{2 k r}^{q}\right)^{1 / q}
$$

where

$$
I_{2 k r}=\left(\left.\int_{\mathbb{R} \times \mathbb{R}^{3}} \sum_{l=0}^{\infty} 2^{\sigma k} e^{-\gamma t} F_{1}^{-1} \psi_{l} \phi_{l} \phi_{k} \frac{1}{s+\mu \xi^{2}} a_{m r} F_{1} f_{r}\right|^{p} d t d x\right)^{\frac{1}{p}} .
$$

Repeating the same considerations as in the proof of Lemma 3.5 we get

$$
\begin{aligned}
I_{2 k r} \leq & c \sum_{l=0}^{\infty} 2^{\sigma(k-l)} 2^{\sigma l} \| \psi_{l}\left(2^{2 l} \xi_{0}, 2^{l} \xi\right) \frac{1}{\gamma+i 2^{2 l} \xi_{0}+\mu\left(2^{l} \xi\right)^{2}} \\
& \cdot a_{m r}\left(2^{2 l} \xi_{0}, 2^{l} \xi\right) \phi_{k}\left(2^{2 l} \xi_{0}, 2^{l} \xi\right)\left\|_{W_{2}^{\frac{d}{2} d}{ }_{\left(\mathbb{R} \times \mathbb{R}^{3}\right)} \cdot\left\|e^{-\gamma x_{0}} F_{1}^{-1} \phi_{k} F_{1} f_{r}\right\|_{L_{p}\left(\mathbb{R} \times \mathbb{R}^{3}\right)}}\right\|\left(\sum_{l=0}^{\infty} \sum_{\sum_{i=1}^{4}\left(s_{i}+2 s_{i}^{0}\right) \leq d}^{s_{1}} \partial_{\xi_{0}}^{s_{1}^{0}} \psi_{l}\right)\left(2^{2 l} \cdot, 2^{l} \cdot\right) \partial_{\xi}^{s_{2}} \partial_{\xi_{0}}^{s_{2}^{0}}\left(\frac{1}{\gamma+i 2^{2 l} \xi_{0}+\mu\left(2^{l} \xi\right)^{2}}\right) \\
& \cdot\left(\partial_{\xi}^{s_{3}} \partial_{\xi_{0}}^{s_{3}^{0}} a_{m r}\right)\left(2^{2 l} \cdot, 2^{l} \cdot\right)\left(\partial_{\xi}^{s_{4}} \partial_{\xi_{0}}^{s_{4}^{0}} \phi_{k}\right)\left(2^{2 l} \cdot, 2^{l} \cdot\right) \|_{L_{2}\left(\mathbb{R} \times \mathbb{R}^{3}\right)},
\end{aligned}
$$

where $d>4$.

By formula (3.10) we obtain

$$
\left|\partial_{\xi}^{s_{2}} \partial_{\xi_{0}}^{s_{2}^{0}}\left(\frac{1}{\gamma+i 2^{2 l} \xi_{0}+\mu\left(2^{l} \xi\right)^{2}}\right)\right| \leq c \frac{2^{l\left(s_{2}+2 s_{2}^{0}\right)}}{|\tau|^{s_{2}+2 s_{2}^{0}+2}},
$$

where $\tau^{2}=\gamma+i 2^{2 l} \xi_{0}+\left(2^{l} \xi\right)^{2}$. Moreover,

$$
\left|\partial_{\xi}^{s_{3}} \partial_{\xi_{0}}^{s_{3}^{0}} a_{m r}\left(2^{2 l} \xi_{0}, 2^{l} \xi\right)\right| \leq c \frac{2^{l\left(s_{3}+2 s_{3}^{0}\right)}}{|\tau|^{s_{3}+2 s_{3}^{0}}} .
$$

Therefore, for $l \neq 0$

$$
\begin{aligned}
& \left|\partial_{\xi}^{s_{2}} \partial_{\xi_{0}}^{s_{2}^{0}}\left(\frac{1}{\gamma+i 2^{2 l} \xi_{0}+\mu\left(2^{l} \xi\right)^{2}}\right)\right| \leq c 2^{-2 l}, \\
& \left|\partial_{\xi}^{s_{3}} \partial_{\xi_{0}}^{s_{3}^{0}} a_{m r}\left(2^{2 l} \xi_{0}, 2^{l} \xi\right)\right| \leq c \quad \text { for }\left(\xi_{0}, \xi\right) \in A,
\end{aligned}
$$

where $a=\left\{\left(\xi_{0}, \xi\right): \frac{1}{4} \leq\left|\left(\xi_{0}, \xi\right)\right|_{a} \leq 4\right\}$, and for $l=0$

$$
\left|\partial_{\xi}^{s_{2}} \partial_{\xi_{0}}^{s_{2}^{0}}\left(\frac{1}{\gamma+i 2^{2 l} \xi_{0}+\mu\left(2^{l} \xi\right)^{2}}\right)\right|+\left|\partial_{\xi}^{s_{3}} \partial_{\xi_{0}}^{s_{3}^{0}} a_{m r}\left(2^{2 l} \xi_{0}, 2^{l} \xi\right)\right| \leq c(\gamma) \quad \text { for }\left(\xi_{0}, \xi\right) \in A
$$

where $A=\left\{\left(\xi_{0}, \xi\right):\left|\left(\xi_{0}, \xi\right)\right|_{a} \leq 4\right\}$.

The derivatives of $\psi_{l}$ and $\phi_{k}$ are estimated as in Lemma 3.6.

By the above considerations we have

$$
I_{2 k r} \leq c \sum_{l=0}^{\infty} 2^{\left(\sigma+d+\frac{5}{2}-L\right)|l-k|} 2^{(\sigma-2) l}\left\|e^{-\gamma x_{0}} F_{1}^{-1} \phi_{l} F_{1} f_{r}\right\|_{L_{p}\left(\mathbb{R} \times \mathbb{R}^{3}\right)} .
$$


Next, the Hölder inequality implies

$$
\begin{aligned}
& \left.\left\|u_{m}\right\|_{\substack{B_{p}^{\frac{\sigma}{2}, \sigma}, \gamma \\
\text { (R) }}} \times \mathbb{R}^{3}\right) \\
& \leq c\left\{\sum_{k=0}^{\infty}\left|\sum_{l=0}^{\infty} \sum_{r=1}^{3} 2^{(\delta-L)|l-k|} 2^{(\sigma-2) l}\left\|e^{-\gamma x_{0}} F_{1}^{-1} \phi_{l} F_{1} f_{r}\right\|_{L_{p}\left(\mathbb{R} \times \mathbb{R}^{3}\right)}\right|^{q}\right\}^{1 / q} \\
& \leq c\left(\sum_{k=0}^{\infty} \sum_{l=0}^{\infty} \sum_{r=1}^{3} 2^{(\delta+\varepsilon-L)|l-k| q} 2^{(\sigma-2) l q}\left\|e^{-\gamma t} F_{1}^{-1} \phi_{l} F_{1} f_{r}\right\|_{L_{p}\left(\mathbb{R} \times \mathbb{R}^{3}\right)}^{q}\right)^{1 / q},
\end{aligned}
$$

where $\delta=\sigma+d+\frac{5}{2}, t=x_{0}, \varepsilon>0$ is arbitrarily small. Assuming that $L>\delta+\varepsilon$ we obtain

$$
\left\|u_{m}\right\|_{B_{p, q, \gamma}^{\frac{\sigma}{2}, \sigma}\left(\mathbb{R}_{+} \times \mathbb{R}^{3}\right)} \leq c\|f\|_{B_{p, q, \gamma}^{\frac{\sigma}{2}-1, \sigma-1}\left(\mathbb{R}_{+} \times \mathbb{R}^{3}\right)}, \quad m=1,2,3 .
$$

This ends the proof.

An immediate consequence of Lemma 4.3 is the corollary below.

Corollary 4.4. Let $f \in B_{p, q}^{\frac{\sigma}{2}-1, \sigma-2}\left((0, T) \times \mathbb{R}^{3}\right), 2<\sigma \in \mathbb{R}, p, q \in(1, \infty), 0<T<\infty$. Assume also that $f^{\prime} \in B_{p, q}^{\frac{\sigma}{2}-1, \sigma-2}\left((-\infty, T) \times \mathbb{R}^{3}\right)$, where

$$
f^{\prime}= \begin{cases}f & \text { for } t \in(0, T), \\ 0 & \text { for } t<0 .\end{cases}
$$

Then there exists a unique solution $u \in B_{p, q}^{\frac{\sigma}{2}, \sigma}\left((0, T) \times \mathbb{R}^{3}\right)$ of the problem

$$
\begin{array}{ll}
u_{t}-\operatorname{div} \mathbb{D}(u)=f & \text { in }(0, T) \times \mathbb{R}^{3}, \\
\left.u\right|_{t=0}=0 & \text { in } \mathbb{R}^{3} .
\end{array}
$$

Moreover, $u^{\prime} \in B_{p, q}^{\frac{\sigma}{2}, \sigma}\left((-\infty, T) \times \mathbb{R}^{3}\right)$ and

$$
\left\|u^{\prime}\right\|_{B_{p, q}^{\frac{\sigma}{2}, \sigma}\left((-\infty, T) \times \mathbb{R}^{3}\right)} \leq c\left\|f^{\prime}\right\|_{B_{p, q}^{\frac{\sigma}{2}-1, \sigma-2}\left((-\infty, T) \times \mathbb{R}^{3}\right)},
$$

where $c=c(T)$ is an increasing function of $T$.

\section{Problem (1.1) with homogenous initial condition in a bounded domain.}

Consider the problem

$$
\begin{array}{ll}
L\left(\partial_{t}, \partial_{x}\right) u \equiv u_{t}-\operatorname{div} \mathbb{D}(u)=f & \text { in } \Omega^{T}, \\
B_{k} u \equiv \bar{\tau}_{k} \cdot \mathbb{D}(u) \bar{n}=b_{k}, \quad k=1,2 & \text { on } S^{T}, \\
B_{3} u \equiv u \cdot \bar{n}=b_{3} & \text { on } S^{T}, \\
\left.u\right|_{t=0}=0 & \text { in } \Omega,
\end{array}
$$

where $\Omega \subset \mathbb{R}^{3}$ is a bounded domain with boundary $S, 0<T<\infty$.

The main result of this section is the following theorem

Theorem 5.1. Let $\sigma \in R_{+}, p, q \in(1, \infty), S \in C^{\sigma+2}, f \in B_{p, q}^{\frac{\sigma}{2}, \sigma}\left(\Omega^{T}\right)$,

$$
b_{k} \in B_{p, q}^{\left(\sigma+1-\frac{1}{p}\right) / 2, \sigma+1-\frac{1}{p}}\left(S^{T}\right), \quad k=1,2, \quad b_{3} \in B_{p, q}^{\frac{\sigma}{2}+1-\frac{1}{2 p}, \sigma+2-\frac{1}{p}}\left(S^{T}\right) .
$$

Let Assumption 1.1 hold with $f_{0}, b_{0 k}(k=1,2)$ and $b_{03}$ replaced by $f, \tilde{b}_{k}(k=1,2)$ and $\tilde{b}_{3}$. Moreover, assume that in the case of $\sigma>\frac{2}{p}$ the function $f$ and $\tilde{b}_{k} \in B_{p, q}^{\frac{\sigma}{2}+\frac{1}{2}, \sigma+1}\left(\Omega^{\prime T}\right)$, 
$k=1,2, \tilde{b}_{3} \in B_{p, q}^{\frac{\sigma}{2}+1, \sigma+2}\left(\Omega^{\prime T}\right)$, extensions of $b_{k}, k=1,2$, and $b_{3}$ to a neighbourhood $\Omega^{\prime} \subset \mathbb{R}^{3}$ of $S$, satisfy Assumption 1.2. If $\frac{3}{p}-1<\sigma \leq \frac{2}{p}$ we assume that $\tilde{b}_{k}(k=1,2)$ and $\tilde{b}_{3}$ fulfill conditions (1.5) and (1.6), respectively. In the case of $\frac{3}{p}-2<\sigma \leq \frac{3}{p}-1$ we assume only that $\tilde{b}_{3}$ satisfies condition (1.6). Then there exists a unique solution $u \in B_{p, q}^{\frac{\sigma}{2}+1, \sigma+2}\left(\Omega^{T}\right)$ to problem (5.1) such that

$$
\int_{0}^{T} \frac{\left\|\partial_{t}^{m_{4}} u\right\|_{L_{p}\left(\Omega^{t}\right)}^{q}}{t^{1+q \lambda_{4}}} d t<\infty,
$$

where $\lambda_{4}=\lambda_{1}$,

$$
m_{4}= \begin{cases}{\left[\frac{\sigma}{2}+1\right]} & \text { if } \frac{\sigma}{2} \text { is noninteger }, \\ \frac{\sigma}{2} & \text { if } \frac{\sigma}{2} \in \mathbb{N} \cup\{0\} .\end{cases}
$$

Moreover,

$$
\begin{aligned}
& \|u\|_{B_{p, q}^{\frac{\sigma}{2}+1, \sigma+2}\left(\Omega^{T}\right)}+\int_{0}^{T} \frac{\left\|\partial_{t}^{m_{4}} u\right\|_{L_{p}\left(\Omega^{t}\right)}^{q}}{t^{1+q \lambda_{4}}} d t \\
& \leq c\left[\|f\|_{B_{p, q}^{\frac{\sigma}{2}, \sigma}\left(\Omega^{T}\right)}+\sum_{k=1}^{2}\left\|b_{k}\right\|_{B_{p, q}^{\left(\sigma+1-\frac{1}{p}\right) / 2, \sigma+1-\frac{1}{p}}\left(S^{T}\right)}\right. \\
& \quad+\left\|b_{3}\right\|_{B_{p, q}^{\frac{\sigma}{2}+1-\frac{1}{2 p}, \sigma+2-\frac{1}{p}}\left(S^{T}\right)}+\left(\int_{0}^{T} \frac{\left.\left\|\partial_{t}^{m_{1}} f\right\|_{L_{p}\left(\Omega^{t}\right)}^{q} d t\right)^{\frac{1}{q}}}{t^{1+q \lambda_{1}}}\right. \\
& \left.\quad+\sum_{k=1}^{2}\left(\int_{0}^{T} \frac{\left\|\partial_{t}^{m_{2}} b_{k}\right\|_{L_{p}\left(S^{t}\right)}^{q}}{t^{1+q \lambda_{2}}} d t\right)^{\frac{1}{q}}+\left(\int_{0}^{T} \frac{\left\|\partial_{t}^{m_{3}} b_{3}\right\|_{L_{p}\left(S^{t}\right)}^{q}}{t^{1+q \lambda_{3}}} d t\right)^{\frac{1}{q}}\right] .
\end{aligned}
$$

To prove Theorem 5.1 we consider the covering $\left\{\Omega^{(k)}\right\}, k \in \mathfrak{M} \cup \mathfrak{N}$, of $\Omega$ introduced in Section 2 and associate with it two families of functions $\left\{\zeta^{(k)}(x)\right\},\left\{\eta^{(k)}(x)\right\}$ such that $\left\{\zeta^{(k)}(x) \eta^{(k)}(x)\right\}$ is a partition of unity (see Section 2).

Let $f^{(k)}(t, x)=\zeta^{(k)}(x) f(t, x)$. Denote by $R^{(k)}, k \in \mathfrak{M}$, the operator such that

$$
u^{(k)}(t, x)=R^{(k)} f^{(k)}(t, x),
$$

where $u^{(k)}(t, x)$ is a solution to the Cauchy problem

$$
\begin{array}{ll}
L\left(\partial_{t}, \partial_{x}\right) u^{(k)}(t, x)=f^{(k)}(t, x) & \text { in }(0, T) \times \mathbb{R}^{3}, \\
\left.u^{(k)}\right|_{t=0}=0 & \text { in } \mathbb{R}^{3} .
\end{array}
$$

For $k \in \mathfrak{N}$ we denote by $R^{(k)}$ the operator such that

$$
\hat{u}^{(k)}(t, z)=R^{(k)}\left(\hat{f}^{(k)}(t, z), \hat{b}^{(k)}\left(t, z^{\prime}\right)\right), \quad z^{\prime}=\left(z_{1}, z_{2}\right),
$$

where $\hat{b}^{(k)}\left(t, z^{\prime}\right)=\left(\hat{b}_{1}^{(k)}\left(t, z^{\prime}\right), \hat{b}_{2}^{(k)}\left(t, z^{\prime}\right), \hat{b}_{3}^{(k)}\left(t, z^{\prime}\right)\right)$ and $\hat{u}^{(k)}(t, z)$ is a solution to the initialboundary value problem

$$
\begin{array}{ll}
L\left(\partial_{t}, \partial_{z}\right) \hat{u}^{(k)}(t, z)=\hat{f}^{(k)}(t, z) & \text { in }(0, T) \times \mathbb{R}_{+}^{3}, \\
B \hat{u}^{(k)}(t, z)=\hat{b}^{(k)}\left(t, z^{\prime}\right) & \text { on }(0, T) \times\left\{z \in \mathbb{R}^{3}: z_{3}=0\right\}, \\
\left.\hat{u}^{(k)}\right|_{t=0}=0 &
\end{array}
$$


where $B=\left(B_{1}, B_{2}, B_{3}\right)$. Then, the operator $R$ called the regularizer is defined as follows $($ see $[15,19])$ :

$$
R g=\sum_{k \in \mathfrak{M} \cup \mathfrak{N}} \eta^{(k)}(x) u^{(k)}(t, x),
$$

where $g=(f, b), b=\left(b_{1}, b_{2}, b_{3}\right)$,

$$
u^{(k)}(t, z)= \begin{cases}R^{(k)} f^{(k)}(t, x) & \text { for } k \in \mathfrak{M}, \\ Z_{k} R^{(k)}\left(Z_{k}^{-1} f^{(k)}(t, x), Z_{k}^{-1} b^{(k)}(t, x)\right) & \text { for } k \in \mathfrak{N},\end{cases}
$$

and $Z_{k}$ is the operator such that $Z_{k} \hat{v}(t, z)=v(t, x)$.

By Corollaries 4.4, 4.2 and Lemma 2.4 there exist solutions to problems (5.3) and (5.4) such that $u^{(k)^{\prime}} \in B_{p, q}^{\frac{\sigma}{2}+1, \sigma+2}\left((-\infty, T) \times \mathbb{R}^{3}\right)$ and $\hat{u}^{(k)^{\prime}} \in B_{p, q}^{\frac{\sigma}{2}+1, \sigma+2}\left((-\infty, T) \times \mathbb{R}_{+}^{3}\right)$, respectively. Moreover, we have the estimates

$$
\begin{gathered}
\left\|u^{(k)^{\prime}}\right\|_{B_{p, q}^{\frac{\sigma}{2}+1, \sigma+2}\left((-\infty, T) \times \mathbb{R}^{3}\right)} \leq c(T)\left\|f^{(k)^{\prime}}\right\|_{B_{p, q}^{\frac{\sigma}{2}, \sigma}\left((-\infty, T) \times \mathbb{R}^{3}\right)}, \quad k \in \mathfrak{M}, \\
\left\|\hat{u}^{(k)^{\prime}}\right\|_{B_{p, q}^{\frac{\sigma}{2}+1, \sigma+2}\left((-\infty, T) \times \mathbb{R}^{3}\right)} \leq c(T)\left(\left\|\hat{f}^{(k)^{\prime}}\right\|_{B_{p, q}^{\frac{\sigma}{2}, \sigma}\left((-\infty, T) \times \mathbb{R}^{3}\right)}\right. \\
\quad+\sum_{j=1}^{2}\left\|\hat{b}_{j}^{(k)^{\prime}}\right\|_{B_{p, q}^{\left(\sigma+1-\frac{1}{p}\right) / 2, \sigma+1-\frac{1}{p}}\left((-\infty, T) \times \mathbb{R}^{2}\right)} \\
\left.+\left\|\hat{b}_{3}^{(k)^{\prime}}\right\|_{B_{p, q}^{\frac{\sigma}{2}+1-\frac{1}{2 p}, \sigma+2-\frac{1}{p}}\left((-\infty, T) \times \mathbb{R}^{2}\right)}\right), \quad k \in \mathfrak{N},
\end{gathered}
$$

where

$$
v^{\prime}=\left\{\begin{array}{ll}
v & \text { for } t \in(0, T), \\
0 & \text { for } t<0,
\end{array} \quad v \in\left\{u^{(k)}, \hat{u}^{(k)}, f^{(k)}, \hat{f}^{(k)}, \hat{b}_{1}^{(k)}, \hat{b}_{2}^{(k)}, \hat{b}_{3}^{(k)}\right\} .\right.
$$

Introduce the spaces:

$$
\begin{aligned}
& H_{p, q}^{\sigma}(-\infty, T) \equiv B_{p, q}^{\frac{\sigma}{2}, \sigma}((-\infty, T) \times \Omega) \times B_{p, q}^{\frac{\sigma}{2}+\frac{1}{2}-\frac{1}{2 p}, \sigma+1-\frac{1}{p}}((-\infty, T) \times S) \\
& \times B_{p, q}^{\frac{\sigma}{2}+\frac{1}{2}-\frac{1}{2 p}, \sigma+1-\frac{1}{p}}((-\infty, T) \times S), \\
& H_{p, q}^{\sigma} \equiv\left\{\left(f, b_{1}, b_{2}, b_{3}\right) \in B_{p, q}^{\frac{\sigma}{2}, \sigma}\left(\Omega^{T}\right) \times B_{p, q}^{\frac{\sigma}{2}+\frac{1}{2}-\frac{1}{2 p}, \sigma+1-\frac{1}{p}}\left(S^{T}\right) \times B_{p, q}^{\frac{\sigma}{2}+\frac{1}{2}-\frac{1}{2 p}, \sigma+1-\frac{1}{p}}\left(S^{T}\right)\right. \\
&\left.\times B_{p, q}^{\frac{\sigma}{2}+1-\frac{1}{2 p}, \sigma+2-\frac{1}{p}}\left(S^{T}\right):\left(f^{\prime}, b_{1}^{\prime}, b_{2}^{\prime}, b_{3}^{\prime}\right) \in H_{p, q}^{\sigma}(-\infty, T)\right\}, \\
& V_{p, q}^{\sigma} \equiv\left\{u \in B_{p, q}^{\frac{\sigma}{2}+1, \sigma+2}\left(\Omega^{T}\right): u^{\prime} \in B_{p, q}^{\frac{\sigma}{2}+1, \sigma+2}((-\infty, T) \times \Omega)\right\}
\end{aligned}
$$

with the norms:

$$
\begin{aligned}
& \left\|\left(f, b_{1}, b_{2}, b_{3}\right)\right\|_{H_{p, q}^{\sigma}}=\left\|\left(f^{\prime}, b_{1}^{\prime}, b_{2}^{\prime}, b_{3}^{\prime}\right)\right\|_{H_{p, q}^{\sigma}(-\infty, T)}, \\
& \|u\|_{V_{p, q}^{\sigma}}=\left\|u^{\prime}\right\|_{B_{p, q}^{\frac{\sigma}{2}+1, \sigma+2}((-\infty, T) \times \Omega)} .
\end{aligned}
$$

Then by (5.5)-(5.6) the following lemma holds.

Lemma 5.2. Let $\sigma \in \mathbb{R}_{+}, p, q \in(1, \infty), S \in C^{\sigma+2}, g \in H_{p, q}^{\sigma}$. Then the regularizer $R: H_{p, q}^{\sigma} \rightarrow V_{p, q}^{\sigma}$ is a bounded linear operator, i.e.

$$
\|R g\|_{V_{p, q}^{\sigma}} \leq c\|g\|_{H_{p, q}^{\sigma}},
$$

where $c>0$ does not depend on $g$. 
Problem (4.1) can be written as

$$
A u=g, \quad A=(L, B) .
$$

Now, our aim is to show the existence of left and right inverse operators to $A$ generated by $R$.

First, we prove

Lemma 5.3. Let $\sigma \in \mathbb{R}_{+}, p, q \in(1, \infty), S \in C^{\sigma+2}, g \in H_{p, q}^{\sigma}$. Then

$$
A R g=g+S g,
$$

where $S$ is a bounded operator in $H_{p, q}^{\sigma}$ with small norm for small $\lambda=\sup _{k} \operatorname{diam} \Omega^{(k)}$ and small T. More precisely,

$$
\|S g\|_{H_{p, q}^{\sigma}} \leq c\left(\varepsilon^{\delta_{1}}+c_{1}\left(\frac{1}{\varepsilon}, \frac{1}{\lambda}\right) T^{\delta_{2}}+c_{2}\left(\|F\|_{C^{\sigma+2}}\right) \lambda^{\delta_{3}}\right)\|g\|_{H_{p, q}^{\sigma}},
$$

where $\varepsilon \in(0,1), c_{1}$ is an increasing positive function of its arguments; $c_{2}$ is a polynomial of the second order and $\delta_{i}>0, i=1,2,3$.

Proof. Exactly as in [27] we have

$$
A R g=\left(S_{1} g+f, S_{2} g+b\right),
$$

where

$$
\begin{aligned}
S_{1} g= & \sum_{k \in \mathfrak{M} \cup \mathfrak{N}}\left(L\left(\partial_{t}, \partial_{x}\right) \eta^{(k)} u^{(k)}-\eta^{(k)} L\left(\partial_{t}, \partial_{x}\right) u^{(k)}\right) \\
& +\sum_{k \in \mathfrak{N}} \eta^{(k)} Z_{k}\left(L\left(\partial_{t}, \partial_{z}-\nabla F \partial_{x_{3}}\right)-L\left(\partial_{t}, \partial_{z}\right)\right) Z_{k}^{-1} u^{(k)}(t, x),
\end{aligned}
$$

$$
S_{2} g=0 \text {. }
$$

Hence $A R g=g+S g$, where $S g=\left(S_{1} g, S_{2} g\right)=\left(S_{1} g, 0\right)$.

First, we consider

$$
\begin{aligned}
& \left\|\sum_{k \in \mathfrak{M} \cup \mathfrak{N}}\left(L\left(\partial_{t}, \partial_{x}\right) \eta^{(k)} u^{(k)^{\prime}}-\eta^{(k)} L\left(\partial_{t}, \partial_{x}\right) u^{(k)^{\prime}}\right)\right\|_{B_{p, q}^{\frac{\sigma}{2}, \sigma}((-\infty, T) \times \Omega)} \\
& \leq \sum_{k \in \mathfrak{M} \cup \mathfrak{N}}\left(c\left(\frac{1}{\lambda}\right)\left\|u_{x}^{(k)^{\prime}}\right\|_{B_{p, q}^{\frac{\sigma}{2}, \sigma}\left((-\infty, T) \times \Omega^{(k)}\right)}\right. \\
& \left.\quad+c\left(\frac{1}{\lambda}\right)\left\|u^{(k)}\right\|_{B_{p, q}^{\frac{\sigma}{2}, \sigma}\left((0, T) \times \Omega^{(k)}\right)}\right), \quad \text { where } u_{x}=\left[u_{i x_{j}}\right]_{i, j=1,2,3} .
\end{aligned}
$$

We have

$$
\begin{aligned}
& \left\|u_{x}^{(k)^{\prime}}\right\|_{B_{p}^{\frac{\sigma}{2}, \sigma}\left(\left((-\infty, T) \times \Omega^{(k)}\right)\right.}=\left\|u_{x}^{(k)}\right\|_{L_{p}\left((0, T) \times \Omega^{(k)}\right)} \\
& +\sum_{i=1}^{3}\left(\int_{0}^{h_{0}} \frac{\left\|\Delta_{i}^{m}(h, \Omega) u_{x}^{(k)}\right\|_{L_{p}\left((0, T) \times \Omega^{(k)}\right)}^{q}}{h^{1+q \sigma}} d h\right)^{1 / q} \\
& \quad+\left(\int_{0}^{h_{0}} \frac{\left\|\Delta_{t}^{m_{0}}(h,(-\infty, T)) u_{x}^{(k)^{\prime}}\right\|_{L_{p}((-\infty, T)) \times \Omega^{(k)}}^{q}}{h^{1+q \frac{\sigma}{2}}} d h\right)^{1 / q} \\
& \equiv K_{1}+K_{2}+K_{3},
\end{aligned}
$$

where we assume that $m>\sigma, m_{0}>\frac{\sigma}{2}+1$ and $m h_{0}<\lambda, m_{0} h_{0}<T, \lambda=\sup _{k} \operatorname{diam} \Omega^{(k)}$. 
First, we estimate

$$
\begin{aligned}
K_{1} & \leq \varepsilon^{1-\varkappa_{1}}\left(\int_{0}^{T}\left\|u^{(k)}\right\|_{W_{p}^{2}\left(\Omega^{(k)}\right)} d t\right)^{1 / p}+c \varepsilon^{-\varkappa_{1}}\left(\int_{0}^{T}\left\|u^{(k)}\right\|_{L_{p}\left(\Omega^{(k)}\right)}^{p} d t\right)^{1 / p} \\
& \leq c \varepsilon^{1-\varkappa_{1}}\left(\int_{0}^{T}\left\|u^{(k)}\right\|_{B_{p, q}^{2+\sigma}\left(\Omega^{(k)}\right)}^{p} d t\right)^{1 / p}+c \varepsilon^{-\varkappa_{1}}\left(\int_{0}^{T}\left\|\int_{0}^{t} u_{\tau}^{(k)}(\tau) d \tau\right\|_{L_{p}\left(\Omega^{(k)}\right)}^{p}\right)^{1 / p} \\
& \leq c \varepsilon^{1-\varkappa_{1}}\left\|u^{(k)}\right\|_{B_{p}^{\frac{\sigma}{2}+1, \sigma+2}\left((0, T) \times \Omega^{(k)}\right)}+c \varepsilon^{-\varkappa_{1}} T\left(\int_{0}^{T}\left\|u_{t}^{(k)}(t)\right\|_{L_{p}\left(\Omega^{(k)}\right)}^{p} d t\right)^{1 / p} \\
& \leq c\left(\varepsilon^{1-\varkappa_{1}}+T \varepsilon^{-\varkappa_{1}}\right)\left\|u^{(k)^{\prime}}\right\|_{B_{p, q}^{\frac{\sigma}{2}+1, \sigma+2}\left((-\infty, T) \times \Omega^{(k)}\right)}
\end{aligned}
$$

where $0<\varkappa_{1}<1$ and we have used the imbedding lemma, the interpolation inequality and the fact that $\left.u^{(k)}\right|_{t=0}=0$. Next, we have

$$
\begin{aligned}
K_{2} \leq & \varepsilon^{1-\varkappa_{2}} \sum_{i=1}^{3}\left(\int_{0}^{h_{0}} \frac{\left(\int_{0}^{T}\left\|\Delta_{i}^{m}(h, \Omega) u^{(k)}\right\|_{W_{p}^{2}\left(\Omega^{(k)}\right)} d t\right)^{q / p}}{h^{1+q \sigma}}\right]^{1 / q} \\
& +c \varepsilon^{-\varkappa_{2}} \sum_{i=1}^{3}\left[\int_{0}^{h_{0}} \frac{\left(\int_{0}^{T}\left\|\int_{0}^{t} \Delta_{i}^{m}(h, \Omega) u_{\tau}^{(k)} d \tau\right\|_{L_{p}\left(\Omega^{(k)}\right)}^{p} d t\right)^{q / p}}{h^{1+q \sigma}}\right]^{1 / q} \\
\leq & c \varepsilon^{1-\varkappa_{2}}\left\|u^{(k)}\right\|_{B_{p}^{\frac{\sigma}{2}+1, \sigma+2}\left((0, T) \times \Omega^{(k)}\right)} \\
& +c \varepsilon^{-\varkappa_{2}} T \sum_{i=1}^{3}\left[\int_{0}^{h_{0}} \frac{\left(\int_{0}^{T}\left\|\Delta_{i}^{m}(h, \Omega) u_{\tau}^{(k)} d \tau\right\|_{L_{p}\left(\Omega^{(k)}\right)}^{p}\right)^{q / p}}{h^{1+q \sigma}}\right]^{1 / q} \\
\leq & c\left(\varepsilon^{1-\varkappa_{2}}+\varepsilon^{-\varkappa_{2}} T\right)\left\|u^{(k)^{\prime}}\right\|_{B_{p, q}^{\frac{\sigma}{2}+1, \sigma+2}\left((-\infty, T) \times \Omega^{(k)}\right)},
\end{aligned}
$$

where $0<\varkappa_{2}<1$ and we used that $\left.u^{(k)^{\prime}}\right|_{t=0}=0$. Finally,

$$
\begin{aligned}
K_{3} & \leq h_{0}^{1 / 2}\left(\int_{0}^{h_{0}} \frac{\left.\left\|\Delta_{t}^{m_{0}}(h,(-\infty, T)) u_{x}^{(k)^{\prime}}\right\|_{L_{p}\left((-\infty, T) \times \Omega^{(k)}\right)}^{q} d h\right)^{1 / q}}{h^{1+q\left(\frac{\sigma}{2}+\frac{1}{2}\right)}}\right. \\
& \leq c T^{1 / 2}\left\|u^{(k)^{\prime}}\right\|_{B_{p, q}^{1+\frac{\sigma}{2}, 2+\sigma}\left((-\infty, T) \times \Omega^{(k)}\right)} .
\end{aligned}
$$

Taking into account the estimates of $K_{1}, K_{2}, K_{3}$ we obtain

$$
\left\|u_{x}^{(k)^{\prime}}\right\|_{B_{p, q}^{\frac{\sigma}{2}, \sigma}\left((-\infty, T) \times \Omega^{(k)}\right)} \leq c\left(\varepsilon^{1-\varkappa}+T^{1 / 2}+T \varepsilon^{-\varkappa}\right) \cdot\left\|u^{(k)^{\prime}}\right\|_{B_{p, q}^{\frac{\sigma}{2}+1, \sigma+2}\left((-\infty, T) \times \Omega^{(k)}\right)} .
$$

Similarly, we estimate $\left\|u^{(k)}\right\|_{B_{p, q}^{\frac{\sigma}{2}, \sigma}\left((0, T) \times \Omega^{(k)}\right)}$. Next, we have

$$
\begin{aligned}
& \left\|\sum_{k \in \mathfrak{N}} \eta^{(k)} Z_{k}\left(L\left(\partial_{t}, \partial_{z}-\nabla F \partial_{x_{3}}\right)-L\left(\partial_{t}, \partial_{z}\right)\right) Z_{k}^{-1} u^{(k)^{\prime}}(t, x)\right\|_{B_{p, q}^{\frac{\sigma}{2}, \sigma}((-\infty, T) \times \Omega)} \\
& \leq c \sum_{k \in \mathfrak{N}}\left\|\left.F_{y} \hat{u}_{z z}^{(k)^{\prime}}\right|_{z=\Phi_{k}(y(x))}\right\|_{B_{p, q}^{\frac{\sigma}{2}, \sigma}\left((-\infty, T) \times \Omega^{(k)}\right)} \\
& \quad+\left\|\left.F_{y} F_{y} \hat{u}_{z z}^{(k)^{\prime}}\right|_{z=\Phi_{k}(y(x))}\right\|_{B_{p, q}^{\frac{\sigma}{2}, \sigma}\left((-\infty, T) \times \Omega^{(k)}\right)}
\end{aligned}
$$




$$
\begin{aligned}
& +\left\|\left.F_{y y} \hat{u}_{z}^{(k)^{\prime}}\right|_{z=\Phi_{k}(y(x))}\right\|_{B_{p, q}^{\frac{\sigma}{2}, \sigma}\left((-\infty, T) \times \Omega^{(k)}\right)}+\left\|\left.F_{y} F_{y y} \hat{u}_{z}^{(k)^{\prime}}\right|_{z=\Phi_{k}(y(x))}\right\|_{B_{p, q}^{\frac{\sigma}{2}, \sigma}\left((-\infty, T) \times \Omega^{(k)}\right)} \\
\leq & c \lambda^{\bar{a}} \sup _{k} \bar{c}\left(\|F\|_{C^{\sigma+2}\left(\Omega^{(k)}\right)}\right) \sum_{k \in \mathfrak{N}}\left\|u^{(k)^{\prime}}\right\|_{B_{p, q}^{\frac{\sigma}{2}+1, \sigma+2}\left((-\infty, T) \times \Omega^{(k)}\right)},
\end{aligned}
$$

where $\bar{a}>0$, and $\bar{c}$ is a polynomial of degree 2 .

The above estimates and inequalities (5.8)-(5.9) imply the assertion of the lemma.

We also need

Lemma 5.4. Let $\sigma \in \mathbb{R}_{+}, p, q \in(1, \infty), S \in C^{\sigma+2}, v \in V_{p, q}^{\sigma}$. Then

$$
R A v=v+W v,
$$

where $W$ is a bounded operator in $V_{p, q}^{\sigma}$ with a small norm for $\lambda$ and $T$ small. More precisely,

$$
\|W v\|_{V_{p, q}^{\sigma}} \leq c\left(\varepsilon^{\bar{\delta}_{1}}+c_{3}\left(\frac{1}{\varepsilon}, \frac{1}{\lambda}\right) T^{\bar{\delta}_{2}}+c_{4}\left(\|F\|_{C^{\sigma+2}}\right) \lambda^{\bar{\delta}_{3}}\right)\|v\|_{V_{p, q}^{\sigma}},
$$

where $\varepsilon \in(0,1), c_{3}$ is an increasing positive function; $c_{4}$ is a polynomial of degree 2 and $\bar{\delta}_{i}>0, i=1,2,3$.

Proof. As in [27] (see Lemma 4.3) $R A v$ can be represented in the form (5.7), where

$$
\begin{aligned}
W v= & \sum_{k \in \mathfrak{M}} \eta^{(k)} R^{(k)}\left(\zeta^{(k)} L\left(\partial_{t}, \partial_{x}\right)-L\left(\partial_{t}, \partial_{x}\right) \zeta^{(k)}\right) v \\
& +\sum_{k \in \mathfrak{N}} \eta^{(k)} Z_{k} R^{(k)}\left[Z_{k}^{-1}\left(\zeta^{(k)} L\left(\partial_{t}, \partial_{x}\right)-L\left(\partial_{t}, \partial_{x}\right) \zeta^{(k)}\right) v, 0\right] \\
& +\sum_{k \in \mathfrak{N}} \eta^{(k)} Z_{k} R^{(k)}\left[\left(Z_{k}^{-1} L\left(\partial_{t}, \partial_{x}\right)-L\left(\partial_{t}, \partial_{x}\right) Z_{k}^{-1}\right) \zeta^{(k)} v\right. \\
& \left.\left.\left(Z_{k}^{-1} B-B Z_{k}^{-1}\right) \zeta^{(k)} v\right|_{s}\right] \equiv I_{1}+I_{2}+I_{3} .
\end{aligned}
$$

First, we have

$$
\begin{aligned}
& \left\|I_{1}+I_{2}\right\|_{B_{p, q}^{\frac{\sigma}{2}+1, \sigma+2}((-\infty, T) \times \Omega)} \leq c \sum_{k \in \mathfrak{M} \cup \mathfrak{N}}\left(c\left(\frac{1}{\lambda}\right)\left\|v_{x}^{\prime}\right\|_{B_{p, q}^{\frac{\sigma}{2}, \sigma}\left((-\infty, T) \times \Omega^{(k)}\right)}\right. \\
& \left.+c\left(\frac{1}{\lambda}\right)\|v\|_{B_{p, q}^{\frac{\sigma}{2}, \sigma}\left((0, T) \times \Omega^{(k)}\right)}\right) \leq c\left(\varepsilon^{1-\varkappa}+T^{1 / 2}+T \varepsilon^{-\varkappa}\right)\|v\|_{V_{p, q}^{\sigma},},
\end{aligned}
$$

where we applied the same calculations as in the proof of Lemma 5.3.

Next, we obtain

$$
\left\|I_{3}\right\|_{B_{p, q}^{\frac{\sigma}{2}+1, \sigma+2}((-\infty, T) \times \Omega)} \leq c \lambda^{\hat{a}} \sup _{k} \hat{c}\left(\left\|F_{k}\right\|_{C^{\sigma+2}\left(\Omega^{(k)}\right)}\right) \sum_{k \in \mathfrak{N}}\left\|v^{(k)^{\prime}}\right\|_{B_{p, q}^{\frac{\sigma}{2}+1, \sigma+2}\left((-\infty, T) \times \Omega^{(k)}\right)},
$$

where $\hat{a}>0$, and $\hat{c}$ is a polynomial of degree 2 .

By the above considerations, the assertion of the lemma follows.

Proof of Theorem 5.1. Lemmas 5.3-5.4 imply the existence of right and left inverse operators to $A$ which are bounded operators equal to $R(I+S)^{-1}$ and $(I+W)^{-1} R$, respectively. Therefore,

$$
R(I+S)^{-1}=(I+W)^{-1} R=A^{-1} .
$$

This yields the assertion of Theorem 5.1 for $T>0$ sufficiently small. 
In the case of arbitrary $0<T<\infty$ we divide the interval $(0, T)$ into a finite number of subintervals: $(0, \bar{t}),\left(\frac{1}{2} \bar{t}, \frac{3}{2} \bar{t}\right), \ldots,(N \bar{t}, T)$ with sufficiently small $\bar{t}>0$. By using the standard argument (see [15]) we extend the solution step by step from $(0, \bar{t})$ onto $(0, T)$.

This ends the proof.

\section{Problem (1.1) with nonhomogeneous initial condition in a bounded do-} main. This section is devoted to the proofs of Theorems 1.1 and 1.2. Let $u_{0} \in$ $B_{p, q}^{\sigma+2-\frac{1}{p}}(\Omega)$. By Lemma 2.3 there exists a function $\tilde{u}_{0} \in B_{p, q}^{\frac{\sigma}{2}+1, \sigma+2}\left(\Omega^{T}\right)$ such that

$$
\begin{gathered}
\left.\tilde{u}_{0}\right|_{t=0}=u_{0}, \\
\left.\partial_{t}^{k} \tilde{u}_{0}\right|_{t=0}=\left.\partial_{t}^{k-1} f\right|_{t=0}+\operatorname{div} \mathbb{D}\left(\left.\partial_{t}^{k-1} \tilde{u}_{0}\right|_{t=0}\right), \quad k=1,2, \ldots, m, \quad 2 m \leq l_{1}
\end{gathered}
$$

where $l_{1}$ is defined in Assumption 1.2 and

$$
\left\|\tilde{u}_{0}\right\|_{B_{p, q}^{\frac{\sigma}{2}+1, \sigma+2}\left(\Omega^{T}\right)} \leq c\left(\left\|u_{0}\right\|_{B_{p, q}^{\sigma+2-\frac{2}{p}}(\Omega)}+\|f\|_{B_{p, q}^{\frac{\sigma}{2}, \sigma}\left(\Omega^{T}\right)}\right) .
$$

Let $v=u-\tilde{u}_{0}$. Then $v$ is a solution of the problem

$$
\begin{aligned}
& v_{t}-\operatorname{div} \mathbb{D}(v)=f-\left(\tilde{u}_{0 t}-\operatorname{div} \mathbb{D}\left(\tilde{u}_{0}\right)\right) \equiv f_{0}, \\
& \left.\bar{\tau}_{\alpha} \cdot \mathbb{D}(v) \bar{n}\right|_{S}=\left.\left(\tilde{b}_{\alpha}-\bar{\tau} \cdot \mathbb{D}\left(\tilde{u}_{0}\right) \bar{n}\right)\right|_{S} \\
& =b_{\alpha}-\left.\left.\bar{\tau}_{\alpha} \cdot \mathbb{D}\left(\tilde{u}_{0}\right)\right|_{S} \cdot \bar{n} \equiv b_{0 \alpha}\right|_{S}, \quad \alpha=1,2, \\
& v \cdot \bar{n}=\left.\left(\tilde{b}_{3}-\tilde{u}_{0} \cdot \bar{n}\right)\right|_{S}=b_{3}-\left.\left.\tilde{u}_{0}\right|_{S} \cdot \bar{n} \equiv b_{03}\right|_{S}, \\
& \left.v\right|_{t=0}=0 .
\end{aligned}
$$

By (6.1)

$$
\begin{aligned}
\left\|f_{0}\right\|_{B_{p, q}^{\frac{\sigma}{2}, \sigma}\left(\Omega^{T}\right)} & \leq c\left(\|f\|_{B_{p, q}^{\frac{\sigma}{2}, \sigma}\left(\Omega^{T}\right)}+\left\|\tilde{u}_{0}\right\|_{B_{p, q}^{\frac{\sigma}{2}+1, \sigma+2}\left(\Omega^{T}\right)}\right) \\
& \leq c\left(\|f\|_{B_{p, q}^{\frac{\sigma}{2}, \sigma}\left(\Omega^{T}\right)}+\left\|u_{0}\right\|_{B_{p, q}^{\sigma+2-\frac{2}{p}}(\Omega)}\right), \\
\left\|\left.b_{0 \alpha}\right|_{S}\right\|_{B_{p, q}^{\left(\sigma+1-\frac{1}{p}\right) / 2, \sigma+1-\frac{1}{p}}\left(S^{T}\right)} & \leq c\left(\left\|b_{\alpha}\right\|_{B_{p, q}^{\left(\sigma+1-\frac{1}{p}\right) / 2, \sigma+1-\frac{1}{p}}\left(S^{T}\right)}+\left\|\tilde{u}_{0}\right\|_{B_{p, q}^{\frac{\sigma}{2}+1, \sigma+2}\left(\Omega^{T}\right)}\right) \\
& \leq c\left(\left\|b_{\alpha}\right\|_{B_{p, q}^{\left(\sigma+1-\frac{1}{p}\right) / 2, \sigma+1-\frac{1}{p}}\left(S^{T}\right)}\right. \\
& \left.\quad+\left\|u_{0}\right\|_{B_{p, q}^{\sigma+2-\frac{2}{p}}(\Omega)}+\|f\|_{B_{p, q}^{\frac{\sigma}{2}, \sigma}\left(\Omega^{T}\right)}\right), \quad \alpha=1,2
\end{aligned}
$$

and

$$
\begin{aligned}
& \left\|b_{03} \mid S\right\|_{B_{p, q}^{\frac{\sigma}{2}+1-\frac{1}{2 p}, \sigma+2-\frac{1}{p}}\left(S^{T}\right)} \\
& \quad \leq c\left(\left\|b_{3}\right\|_{B_{p, q}^{\frac{\sigma}{2}+1-\frac{1}{2 p}, \sigma+2-\frac{1}{p}}\left(S^{T}\right)}+\left\|u_{0}\right\|_{B_{p, q}^{\frac{\sigma}{2}+2-\frac{2}{p}}(\Omega)}+\|f\|_{B_{p, q}^{\frac{\sigma}{2}, \sigma}\left(\Omega^{T}\right)}\right) .
\end{aligned}
$$

Therefore, Theorem 5.1 implies the existence of a unique solution $v \in B_{p, q}^{\frac{\sigma}{2}+1, \sigma+2}\left(\Omega^{T}\right)$ of problem (6.3), and this yields the existence of a solution of problem (1.1).

The uniqueness of $u$ follows from (1.7), while the estimate (1.7) is a consequence of (6.3)-(6.5) and estimate (5.2) for $v$.

This ends the proof of Theorem 1.1 .

Theorem 1.2 is an immediate corollary of Theorem 1.1 and Lemmas 2.3 and 2.5. 


\section{References}

[1] M. S. Agranovich and M. I. Vishik, Elliptic problems with parameter and parabolic problems of general type, Usp. Mat. Nauk 19 (117) (1964), no. 3, 53-161 (in Russian).

[2] W. Alame, On the existence of solutions for the nonstationary Stokles system with slip boundary conditions in general Sobolev-Slobodetskii and Besov spaces, in: Regularity and Other Aspects of the Navier-Stokes Equations, Banach Center Publ. 70 (2005), 21-49.

[3] H. Amann, Dynamic theory of quasilinear parabolic equations. II. Reaction-diffusion systems, Diff. Int. Equs. 3 (1990), 13-75.

[4] H. Amann, Linear and Quasilinear Parabolic Problems, Vol. I, Birkhäuser, 1995.

[5] H. Amann, Elliptic operators with infinite-dimensional state spaces, J. Evol. Equ. 1 (2001), $143-188$

[6] O. V. Besov, V. P Il'in and S. M. Nikolskij, Integral Representations of Functions and Theorems of Imbedding, Nauka, Moscow 1975 (in Russian).

[7] M. Burnat and W. M. Zajączkowski, On local motion of a compressible barotropic viscous fluid with the boundary slip condition, Topol. Meth. Nonlinear Anal. 10 (1997), 195-223.

[8] R. Danchin, Global existence in critical spaces for flows of compressible viscous and heatconductive gases, Archive for Rational Mechanics and Analysis 160 (2001), 1-39.

[9] R. Danchin, On the uniqueness in critical spaces for compressible Navier-Stokes equations, NoDEA 12 (2005), 111-128.

[10] G. Grubb, Functional Calculus of Pseudodifferential Boundary Problems, Progress in Mathematics 65, Birkhäuser, 1996.

[11] G. Grubb and V. A. Solonnikov, Solution of parabolic pseudo-differential initial-boundary value problems, Journal of Differential Equations 87 (1990), 256-304.

[12] G. Grubb and N. J. Kokholm, A global calculus of parameter-dependednt pseudodifferential boundary problems in $L_{p}$ Sobolev spaces, Acta Math. 171 (1993), 165-229.

[13] G. Grubb, Parameter-elliptic and parabolic pseudodifferential boundary problems in global $L_{p}$ Sobolev spaces, Mathematische Zeitschrift 218 (1995), 43-90.

[14] G. Grubb and V. A. Solonnikov, Boundary value problems for the nonstationary Navier-Stokes equations treated by pseudo-differential methods, Math. Scand. 69 (1991), $217-290$

[15] O. A. Ladyzhenskaya, V. A. Solonnikov and N. N. Uraltseva, Linear and Quasilinear Equations of Parabolic Type, Nauka, Moscow, 1967 (in Russian).

[16] J. L. Lions and E. Magenes, Non-Homogeneous Boundary Value Problems and Applications, Springer, 1972.

[17] S. M. Nikolskij, Approximation of Functions of Several Variables and Imbedding Theorems, Nauka, Moscow 1977 (in Russian).

[18] R. Paley and N. Wiener, Fourier Transforms in the Complex Domain, New York, 1934.

[19] V. A. Solonnikov, A priori estimates for linear parabolic equations of the second order, Trudy Mat. Inst. Steklova 70 (1964), 133-212 (in Russian).

[20] V. A. Solonnikov, On boundary value problems for linear parabolic systems of differential equations of general type, Trudy Mat. Inst. Steklova 83 (1965) (in Russian).

[21] V. A. Solonnikov, An initial-boundary value problem for Stokes system that arises in the study of free boundary problems, Trudy Mat. Inst. Steklova 188 (1990), 150-188 (in Russian); English transl.: Proc. Steklov Inst. Math. 3 (1991), 191-239. 
[22] H. Triebel, Interpolation Theory, Function Spaces, Differential Operators, North-Holland, Amsterdam, 1978.

[23] H. Triebel, Theory of Function Spaces, Akad. Verlagsgesellschaft, Leipzig, 1983.

[24] P. Weidemaier, Maximal regularity for parabolic equations with inhomogeneous boundary conditions in Sobolev spaces with mixed $L_{p}$-norm, Electr. Res. Announcements of the AMS 8 (2002), 47-51.

[25] P. Weidemaier, Existence results in $L_{p}-L_{q}$ spaces for second order parabolic equations with inhomogeneous Dirichlet boundary conditions, in: Progress in PDE, Pitman Research Notes 384, 1998, 189-200.

[26] Y. Yamamoto, Solutions in Besov spaces of a class of abstract parabolic equations of higher order in time, J. Math. Kyoto Univ. 38 (1998), 201-227.

[27] E. Zadrzyńska and W. M. Zajączkowski, The Cauchy-Dirichlet problem for the heat equation in Besov spaces, Zapiski Nauchn. Sem. POMI 348 (2007), 40-97. 